Departamento de Inteligencia Artificial

Escuela Técnica Superior de Ingenieros Informáticos

$\mathrm{PhD}$ Thesis

\title{
Crossing the Chasm Between Ontology \\ Engineering and Application Development
}

Author: Paola Patricia Espinoza Arias

Supervisor: Dr. Oscar Corcho García

November, 2021 

Tribunal nombrado por el Sr. Rector Magfco. de la Universidad Politécnica de Madrid, el día ...... de de

Presidente:

Vocal:

Vocal:

Vocal:

Secretario:

Suplente:

Suplente:

Realizado el acto de defensa y lectura de la Tesis el día ...... de de en la Escuela Técnica Superior de Ingenieros Informáticos.

Calificación:

EL PRESIDENTE

VOCAL 1

VOCAL 2

VOCAL 3

EL SECRETARIO 

A mis papis y mi ñaño.

A César. 



\section{Agradecimientos}

Hace un par de años jamás imaginé como iba a cambiar mi vida tras aquella ceremonia en la que el gobierno ecuatoriano me otorgó una beca para estudiar en el extranjero. Fue así que llegué a Madrid, con mucha ilusión, decidida a aprovechar al máximo aquella oportunidad que por años había estado esperando para cursar un máster, y, sobre todo, con muchísimas ganas de aprender. Y fue en una de esas clases del máster que apareció en escena Oscar Corcho, ese profesor e investigador brillante que tanto admiro, el que conecta con sus estudiantes, con el que terminas la clase y las discusiones con ganas de saber más, pero sobre todo ese ser humano maravilloso y optimista, que siempre tiene una sonrisa y una solución para casi todo. Muchísimas gracias Oscar por haberme dado la oportunidad de trabajar contigo, por confiar en mí y animarme a avanzar a pesar de mis inseguridades, por tu apoyo y tu paciencia. Gracias por haberme abierto las puertas del Ontology Engineering Group (OEG), ese grupo que se ha convertido en mi familia, en el que me hicieron sentir como en casa, y en el que he aprendido tanto.

También quiero agradecer de manera muy especial a Dani Garijo por todas las discusiones, los consejos, y por el gran apoyo que me has dado durante este tiempo. Gracias por tu sinceridad y por tus comentarios siempre constructivos que sin duda me han hecho crecer un montón. Eres un gran mentor y me ha enriquecido muchísimo trabajar contigo. Asimismo un agradecimiento especial para María Poveda por compartir tu valioso tiempo y conocimiento conmigo, por escucharme y guiarme cada vez que las dudas me acechaban. Muchísimas gracias por los trabajos que hicimos juntas.

Hacer esta tesis no habría sido lo mismo sin la gente maravillosa que he tenido a mi alrededor. Gracias David, mi muchacho gallego, porque desde el primer día que llegué al OEG hasta ahora has sido un gran apoyo dentro y fuera del laboratorio, y porque tengo la dicha de llamarte amigo. Gracias Carlos, mi hermano mayor, por todo tu apoyo y consejos, y por hacerme creer que "si se quiere se puede". Gracias a los compañeros que estuvieron 
o que todavía están trabajando en el OEG y con los que siempre compartí buenos momentos e interesantes conversaciones: Patri, Alba, Jhon, Paco, Elvi, Serge, Álvaro, Pablo, Filip, Freddy, Nandana, Ana, Julia, Miguel Ángel, Raúl Alcázar, José Luis, María Navas, Andrea, etc. A los investigadores senior como Asun, Lupe, Mariano Fernández, Raúl García, Víctor, y Elena que son inspiración para todos. A J.A.R.G y Ana Ibarrola por su ayuda con todas las gestiones administrativas. Y por último muchísimas gracias a Edna por apoyarme con el trabajo, por estar siempre pendiente de mi y motivarme a seguir adelante.

Gracias también a los amigos que han estado alentandome desde el otro lado del mundo y pendientes de cada uno de mis pasos: Vite, Jessy, Daniel, Adonis y Jessy, Joselo, Mariel, Franz, Roque, Chori, Dani y Paula. Y a quienes han estado en este lado del mundo brindadome su ayuda y amistad: Gaby y Luis, Bojan y Nina, Olga y Alex.

Gracias infinitas a César por recorrer junto conmigo este camino, por la paciencia, y por comprenderme. Han sido años intensos, con muchos retos, y en los que más de una vez he querido tirar la toalla, pero has sido tú quien ha estado a mi lado en cada paso, pendiente de mi en todo momento, siempre con una gran sonrisa y haciendome reír, cuidandome con tanto amor, y ayudandome a surfear las olas. Gracias también a Paqui y Lucas, que me han adoptado como si fuera su hija y me han llenado de cariño y dado aliento en todo este tiempo. Y por supuesto muchísimas gracias también a Prado, Alberto, Eva, e Inés por su apoyo y por darme ánimos.

Finalmente, quiero agradecer a toda mi familia. De manera especial gracias a mis papis, por brindarme tanto cariño y apoyo en todo momento de mi vida, y por sus sabios consejos. Pero sobre todo gracias por saber entender mis decisiones y por animarme siempre a seguir adelante y a luchar por mis sueños. Sin ustedes nada de esto hubiera sido posible, y aunque estemos lejos la distancia solo la ponen los kilómetros porque el amor que les tengo es más inmenso que eso. Gracias también a mi ñaño porque, aunque seamos tan diferentes, sabemos el gran cariño que nos une, y que nos tenemos siempre el uno al otro para lo que sea en las buenas y en las malas. 


\begin{abstract}
Nowadays there is a large number of Knowledge Graphs (KGs) that have been generated by public and private organizations in order to integrate their data, and facilitate their interoperability. In this context, ontologies are fundamental since they provide the structure with which data are organized in KGs. However, ontologies have generally not been taken into account when designing and generating Application Programming Interfaces (APIs) to allow developers to consume KG data in a developer-friendly way. In addition, existing ontology development methodologies have not detailed how ontologies should be used after their publication and specifically how application developers could use them.

This thesis addresses the challenge of facilitating the consumption of KG data by application developers who are not ontology experts. To this end, this thesis proposes a new phase, called ontology exploitation, to be considered at the end of the ontology development process. More specifically, within the ontology exploitation phase a method for the generation of APIs from the artefacts generated during the ontology development is proposed. This method details the activities and tasks that must be carried out to design and generate APIs reusing these ontology artefacts. In addition, to support some of the tasks of this method, this thesis presents a tool that allows APIs to be generated from the competency questions and ontology serialization.

The conclusions of this thesis reflect that the limitations found in the state of the art have been addressed both at the methodological, technological and tooling levels for the generation of APIs based on ontology artefacts. Finally, the lines of future work reflect several research challenges that need to be addressed so that the great potential of KGs and ontologies is finally exploited by application developers.
\end{abstract}





\section{Resumen}

Hoy en día existe una gran cantidad de Knowledge Graphs (KGs) que han sido generados por organizaciones públicas y privadas con la finalidad de integrar sus datos, y facilitar su interoperabildad. En este contexto, las ontologías son fundamentales ya que proveen la estructura con la que se organizan los datos en los KGs. Sin embargo, las ontologías generalmente no se han tomado en cuenta cuando se diseñan y generan Application Programming Interfaces (APIs) para permitir que los desarrolladores consuman los datos de los KGs de una forma amigable. Asimismo, las metodologías de desarrollo de ontologías existentes no han detallado cómo deben utilizarse tras su publicación y concretamente cómo los desarrolladores de aplicaciones podrían utilizarlas.

En esta tesis se aborda el reto de facilitar el consumo de datos de KGs a los desarrolladores de aplicaciones que no son expertos en ontologías. Para ello, esta tesis propone una nueva fase, denominada explotación de la ontología, a ser considerada al final del proceso de desarrollo ontologías. Concretamente, dentro de la fase de explotación de la ontología se propone un método para la generación de APIs a partir de los artefactos que se generan durante el desarrollo de ontologías. Este método detalla las actividades y tareas que deben llevarse a cabo para diseñar y generar APIs reusando estos artefactos de ontologías. Además, para dar soporte a algunas de las tareas de este método, esta tesis presenta una herramienta que permite generar APIs a partir de las preguntas de competencia y la serialización de la ontología.

Las conclusiones de esta tesis reflejan que se ha abordado las limitaciones encontradas en el estado del arte tanto al nivel metodológico como technológico y de herramientas para la generación de APIs basadas en artefactos de ontologías. Finalmente, las líneas de trabajo futuro reflejan varios desafíos de investigación que requieren ser abordados para que el gran potencial que tienen los KGs y las ontologías sea finalmente explotados por los desarrolladores de aplicaciones. 



\section{Contents}

Acronyms

1 Introduction 1

1.1 Thesis structure . . . . . . . . . . . . . . . . . 3

1.2 Related publications . . . . . . . . . . . . . . 4

2 State of the Art

2.1 Terminology .......................... 7

2.2 Methodologies for ontology development . . . . . . . . . . . 8

2.2.1 Methodology by Grüninger and Fox . . . . . . . . . . 8

2.2 .2 METHONTOLOGY . . . . . . . . . . . . . . . . 10

2.2 .3 On-To-Knowledge . . . . . . . . . . . . . . . . 11

2.2 .4 NeOn Methodology . . . . . . . . . . . . . . . . . . . 12

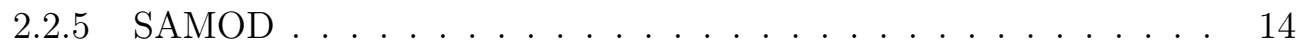

2.2 .6 LOT . . . . . . . . . . . . . . . . . . . . 15

2.3 Approaches for API generation from ontologies . . . . . . . . . . 16

2.3 .1 Specifications . . . . . . . . . . . . . . . 17

2.3.1.1 SPARQL Protocol . . . . . . . . . . . . . . 17

2.3.1.2 SPARQL 1.1 Graph Store Protocol (GSP) . . . . . . . 17

2.3.1.3 Linked Data API (LDA) . . . . . . . . . . . 17

2.3.1.4 Hydra Vocabulary . . . . . . . . . . . . . 18

2.3.1.5 Linked Data Platform (LDP) . . . . . . . . . 18

2.3.1.6 Linked Data Templates (LDT) . . . . . . . . . 18

2.3.1.7 Social Linked Data specification (Solid) . . . . . . . . 19

2.3.1.8 OpenAPI Specification (OAS) . . . . . . . . . 19 
2.3.1.9 GraphQL . . . . . . . . . . . . . . . . . . . 19

2.3.2 Technologies and Tools . . . . . . . . . . . . . . . . 19

$2.3 .2 .1 \quad$ KG stores . . . . . . . . . . . . . . . 20

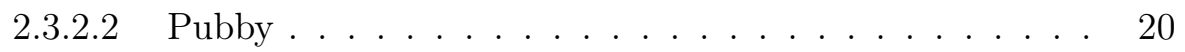

2.3 .2 .3 Puelia ...................... 20

2.3.2.4 Epimorphics Linked Data API Implementation (ELDA) 20

2.3.2.5 Linked Open Data Inspector (LODI) . . . . . . . . . . 21

2.3.2.6 Apache Marmotta . . . . . . . . . . . . . . . . . . 21

2.3.2.7 Building Apis SImpLy (BASIL) _ . . . . . . . . 21

2.3.2.8 Git repository linked data API constructor (GRLC) . . 21

2.3.2.9 AtomGraph Procesor . . . . . . . . . . . 22 2

2.3 .2 .10 JSON-QB API . . . . . . . . . . . . . . . . . 22

2.3.2.11 Open Semantic Framework (OSF) . . . . . . 22

2.3 .2 .12 Trellis . . . . . . . . . . . . . . . . 22

2.3.2.13 Ontology-Based APIs (OBA) . . . . . . . . 23

2.3.2.14 RESTful-API for RDF data (R4R) . . . . . . . 23

2.3 .2 .15 OWL2OAS . . . . . . . . . . . . . . . 23

2.3.2.16 Ontology2GraphQL . . . . . . . . . . . 23

2.3.2.17 Restful API Manager Over SPARQL Endpoints (RAMOSE) 24

2.3.2.18 Community Solid Server . . . . . . . . . . . 24

2.3.2.19 Walder . . . . . . . . . . . . . . . . . 24

2.3.3 Analysis of specifications, technologies and tools for API definition and generation . . . . . . . . . . . . . . 25

2.3.3.1 Criteria for comparing API specifications . . . . . 25

2.3.3.2 API specification comparison . . . . . . . . . . 26

2.3.3.3 Criteria for comparing technologies and tools for generating APIs . . . . . . . . . . . . . . 30

2.3.3.4 Results of comparison of API generation technologies and tools ....................... 31

2.4 Conclusions and limitations of the State of the Art . . . . . . . . 38 
3 Objectives and Contributions 41

3.1 Objectives . . . . . . . . . . . . . . . . . 41

3.2 Contributions to the State of the Art . . . . . . . . . . 42 42

3.3 Assumptions . . . . . . . . . . . . . . . . . . . . . . 43

3.4 Hypotheses . . . . . . . . . . . . . . . . . . 43

3.5 Restrictions . . . . . . . . . . . . . . . . . 44

3.6 Evaluation Plan . . . . . . . . . . . . . . . . . . . . 44

3.7 Research methodology . . . . . . . . . . . . . . . . . . . 446

4 A method for API generation based on ontology artefacts 49

4.1 Activities within the API generation method based on ontology artefacts 49

4.1 .1 API design activity . . . . . . . . . . . . . . 51

4.1.1.1 API paradigm selection . . . . . . . . . . 5 52

4.1.1.2 Competency questions analysis . . . . . . . . 5 53

4.1.1.3 Authentication and authorization mechanisms selection 58

4.1 .2 API specification activity $\ldots \ldots \ldots \ldots$

4.1 .3 API implementation activity . . . . . . . . . . 60 60

4.1 .4 API validation activity . . . . . . . . . . . . . . . 61 61

4.1 .5 API deployment activity . . . . . . . . . . . . . . 62 62

4.2 Supporting the API generation process with OATAPI . . . . . . . . 63

4.2 .1 OATAPI's overview . . . . . . . . . . . . . . 63

4.2.2 Analysis of competency questions . . . . . . . . . . . . 6 64

4.2.3 Complementary support for the API generation activities . . . . 66

4.2.3.1 API specification . . . . . . . . . . . . . 6 66

4.2.3.2 API implementation and validation . . . . . . 68

5 Evaluation 69

5.1 Evaluation of indistinguishable API paths . . . . . . . . . . . 69

5.1 .1 Experimental design . . . . . . . . . . . . . . . . . 69

5.1 .2 Results and discussion . . . . . . . . . . . . . 72

5.1.2.1 Background analysis . . . . . . . . . . . 72

5.1.2.2 REST APIs behaviour analysis . . . . . . . . . 74

5.1.2.3 API paths based on ontology artefacts analysis . . . . 75

5.1 .3 Conclusions of the experiment . . . . . . . . . . . . 77 
5.2 Evaluation of similarity between API paths $\ldots \ldots \ldots \ldots$

5.2 .1 Experimental design . . . . . . . . . . . . . 79

5.2.1.1 Manual generation of API paths . . . . . . . . 79

5.2.1.2 Automatic generation of API paths . . . . . . . . 81

5.2.1.3 Similarity comparison . . . . . . . . . . . . . 82

5.2 .2 Results and discussion . . . . . . . . . . . . . . . . 83

5.2 .3 Conclusions of the experiment . . . . . . . . . . . 85

6 Conclusions and future work

6.1 Contributions . . . . . . . . . . . . . . . . . . . . . . . . 87

6.2 Future work . . . . . . . . . . . . . . . . . . . . . . . 89

A REST APIs resource naming strategy 103

A.1 Rules for naming REST APIs . . . . . . . . . . . . . . . . . 103

A.2 Rules for naming REST APIs based on ontology artefacts . . . . . . 105

B API Specification Template $\quad 109$

C Questionnaire on generating REST APIs from ontology artefacts 111

C.1 Background . . . . . . . . . . . . . . . . . . . 112

C.2 Specific questions regarding REST APIs . . . . . . . . . . . . . . 113

C.3 Specific questions on APIs based on ontology artefacts . . . . . . . . . 113

C.3.1 First domain: Local business census and activities licenses. . . . 113

C.3.2 Second domain: Air Quality . . . . . . . . . . . . . . 115

C.3.3 Third domain: Public Procurement . . . . . . . . . . . . . . 117 


\section{List of Figures}

2.1 Procedure for ontology design and evaluation (Grüninger and Fox, 1995) 9

2.2 Ontology development process and life cycle in METHONTOLOGY (GómezPérez et al. 2006) . . . . . . . . . . . . . . . 10

2.3 On-To-Knowledge development process or knowledge metaprocess Staab et al. 2001) . . . . . . . . . . . . . . . . . . 11

2.4 NeOn scenarios for building ontology networks (Suárez-Figueroa et al.,

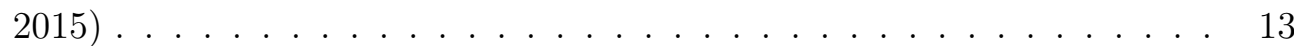

2.5 A brief summary of SAMOD, starting with the "Collect requirements

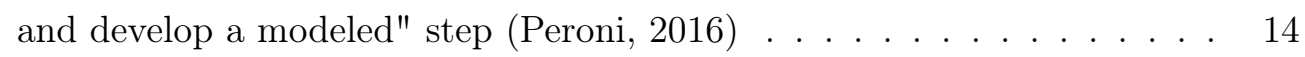

2.6 LOT methodology base workflow of processes (Poveda-Villalón et al., 2019) 16

2.7 Data management levels offered by API generation technologies and tools for $\mathrm{KG}$ consumption. . . . . . . . . . . . . . . . . . . 36

3.1 Relations between objectives, contributions, hypotheses, restrictions and assumptions of this thesis. . . . . . . . . . . . . . . 45

3.2 Phases of the thesis development, including the activities carried out during each phase and the main publications derived from each phase. . 48

4.1 Ontology exploitation phase as an extension of the LOT Methodology. New elements are illustrated in brown. . . . . . . . . . . . . . 50

4.2 Activities of the method to generate APIs based on ontology artefacts (This figure follows the convention defined by the LOT methodology.) . 51

4.3 Tasks involved in the API design activity . . . . . . . . . . . . 52

4.4 Interactions between the client and SPARQL endpoint . . . . . . . . 61 61

4.5 API validation by executing an API call to the server. . . . . . . . . . . 62 
5.1 Number of Web APIs developed per participant . . . . . . . . . . . 73

5.2 Participants' background in semantic technologies, and specifications, tools, and serializations they are used to. . . . . . . . . . . . 74

5.3 Expected answers after executing a GET operation on a API path. . . . 75

5.4 F-measure of automatically generated API routes matching manually generated ones. . . . . . . . . . . . . . . . . 84 


\section{List of Tables}

2.1 Comparison criteria for specifications . . . . . . . . . . 26 26

2.2 Analysis of API Specifications according to our comparison framework. . 28

2.3 Comparison criteria for technologies and tools . . . . . . . . . . . 30

2.4 Analysis of API generation technologies and tools for KG consumption according to our comparison framework. . . . . . . . . . . 33

4.1 Common competency questions terms denoting operations in APIs. . . . 53

4.2 Example of operations detection for counting and boolean queries . . . . 54

4.3 Operations detection example . . . . . . . . . . . . . . 5 54

4.4 Example of ontology elements identification . . . . . . . . . . . 555

4.5 Example of ontology elements identification and API paths generation . 56

4.6 Example of an API specification for the local business census ontology. . 59

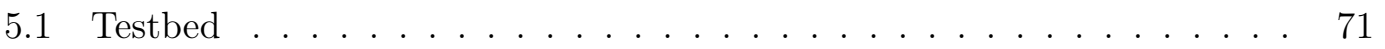

5.2 Familiarity of the participants with the technologies . . . . . . . 73

5.3 Summary of results from the evaluation of API paths manually vs automatically generated . . . . . . . . . . . . . . . . . . . 76

5.4 Summary of results about the participant's preference of API paths . . . 78

5.5 Excerpt of the corpus of the VideoGame ontology CQs. . . . . . . . . 8 80

5.6 Summary of ontologies, CQs manually analyzed, and valid CQs. . . . . 81

5.7 API paths automatically generated with OATAPI . . . . . . . . . . . 82

5.8 Results obtained from the ROUGE metric evaluation of the API paths manually and automatically generated. . . . . . . . . . . . 83 



\section{List of Algorithms}

1 Competency questions analysis ............... 65 



\section{Acronyms}

API: Application Programming Interface

CQ: Competency Question

FAIR: Findable Accessible Interoperable Reusable

GUI: Graphical User Interface

HATEOAS: Hypermedia as the Engine of Application State

IDL: Interface Description Language

JSON: JavaScript Object Notation

KG: Knowledge Graph

NLP: Natural Language Processing

OAS: OpenAPI Specification

OATAPI: Ontology Artefacts to Application Programming Interfaces

ORSD: Ontology Requirement Specification Document

OWL: Web Ontology Language

RDB2RDF: Relational Database to Resource Description Framework

RDF: Resource Description Framework

REST: Representational State Transfer Protocol

ROUGE: Recall-Oriented Understudy for Gisting Evaluation

SPARQL: SPARQL Protocol And RDF Query Language

URI: Uniform Resource Identifier

URL: Uniform Resource Locator 



\section{Chapter 1}

\section{Introduction}

Knowledge Graphs (KGs) have become a crucial asset for structuring data and factual knowledge in private and public organizations. Several prominent KGs have been generated over recent years to improve search capabilities, empower business analytics, ease decision making, etc. (Hogan et al., 2020). Industry KGs have been created by companies like Google, Microsoft, Facebook, eBay, IBM, among others, to make their services "smarter" and add value to users (Noy et al., 2019). Open KGs such as DBpedia (Lehmann et al., 2015) cover a wide variety of domains, and crowdsourced KGs like Wikidata (Vrandečić and Krötzsch, 2014) are actively maintained by an international and diverse community of curators. There are also plenty of examples of domain-specific KGs, which have also used as a way to structure and plublish open data by public administrations of several countries (e.g. national administrations: US (Hendler et al., 2012), UK (Shadbolt et al., 2012), and local administrations: Zaragoza in Spain (Espinoza-Arias et al., 2020a), Bologna in Italy (Corradi et al., 2014)); by libraries (e.g. by the Spanish National Library (Vila-Suero et al., 2013), British (Deliot, 2014), and French (Simon et al., 2013)); by the life sciences community (e.g., the Monarch initiative to integrate data of genes, diseases, phenotypes, variants, and genotypes across species (Shefchek et al., 2020), and DisGeNET (Piñero et al., 2020) to describe data about genes and variants associated to human diseases).

KGs are commonly structured by means of ontologies (Studer et al., 1998). Ontologies are used to structure data without ambiguities, provide shared meaning and infer new knowledge. Ontologies are usually developed following well defined methodologies see some relevant methodologies at (Corcho et al., 2003; Garcia et al., 2010; Keet, 2018, 
Kotis et al., 2020), which recommend a specific development life cycle and describe the set activities and tasks that may be carried out by ontology engineers and many other stakeholders involved in the process (e.g. domain experts, users). In the initial stage of most of these methodologies a set of use cases and competency questions are identified, so as to drive the ontology design. Then, at the implementation stage of the ontology development, usually, one or several files are encoded in a formal ontology implementation language, such as OWL (McGuinness et al., 2004). In addition, if best practices for ontology publishing are followed (Berrueta et al. 2008), such files are published together with their HTML documentation, which providea human-readable descriptions of the corresponding ontologies. Some of these methodologies identify a final stage of maintenance, which considers the evolution and versioning of the ontologies, which may be caused by needed corrections, changes in the original requirements, and updates in the re-used or imported ontologies, among others.

However, despite their adoption, KGs are still challenging to consume by application developers. On the one hand, developers face a production-consumption challenge: there is a gap between the ontology engineers who design an ontology and may intervene in $\mathrm{KG}$ creation and the application developers who want to consume its contents (Fletcher et al. 2020). Ontologies may be complex, and the resources used during their development (use cases, requirements, discussion logs, etc.) are often not made available to their users (e.g. application developers). As a result, developers usually need to duplicate some of the effort already done by ontology engineers when they were understanding the domain, interacting with domain experts, taking modeling decisions, etc. On the other hand, application developers face technical challenges: many of them are not familiar with Semantic Web standards such as OWL and SPARQL (Seaborne and Harris, 2013), and hence those KGs that are exclusively based on Semantic Web technologies remain hardly accessible to them (Verborgh and Vander Sande, 2020). Developers (and in particular web developers) are mostly used to data representation formats like JSON (Bray et al., 2014); and Application Programming Interfaces (APIs) for accessing data. APIs allow the communication and interaction between services without having to provide details about how they are implemented. The de facto architectural style for building APIs is the resource-oriented REpresentational State Transfer (REST) architectural style (Fielding and Taylor, 2002). 
In order to address both production-consumption and technical challenges, multiple approaches have been proposed in recent years by the Semantic Web community, ranging from Semantic RESTful APIs (Salvadori and Siqueira, 2015) which are compatible with Semantic Web and REST; to tools to create Web APIs on top of SPARQL endpoints (Daga et al., 2015; Groth et al., 2014; Meroño-Peñuela and Hoekstra, 2016, Schröder et al. 2018). Outside the Semantic Web community, approaches like GraphQL (GraphQL, Foundation, 2015) are gaining traction among developers due to their flexibility to query and retrieve data from public endpoints. However, we have not identified so far any work focused on generating KG consumption APIs from the ontology artefacts generated during the ontology development process.

The main goal of this thesis is to advance in this direction facilitating the consumption of ontology-based KGs by application developers who are not experts in ontologies, while reusing some of those intermediate products of the ontolgy development process. More precisely, two objectives have been defined: a) help ontology engineers generate APIs to allow ontology usage and KG data consumption by application developers, b) automate API generation from ontology artefacts generated during the ontology development process. To that end, the main contributions of this thesis are: a) a method for ontology-based API generation, which proposes a set of activities and tasks to define, specify, implement, validate, and deploy APIs based on ontology artefacts, and b) a tool for supporting API generation (OATAPI), which allows generating a set of APIs and SPARQL queries based on two ontology artefacts: competency questions and ontology implementation.

\subsection{Thesis structure}

The thesis is structured as follows:

- Chapter 2 provides a general vision of the existing work in the areas related to this thesis. This chapter is divided into two parts. The first one presents the most relevant methodologies for ontology development. The second one provides the main approaches for API generation that have been proposed in the Semantic Web field. Finally, some conclusions are given, with a special focus on the limitations of current approaches. 
- Chapter 3 defines the objectives, contributions, assumptions, hypotheses and restrictions for this thesis. This chapter also includes the research methodology followed and the evaluation plan of the hypotheses.

- Chapter 4 describes our method to define, specify, implement, validate, and deploy APIs based on ontology artefacts. The chapter also presents a tool that we have developed to provide support to this method, automating some of its tasks.

- Chapter 5 presents the evaluation of the main hypotheses of the thesis.

- Chapter 6 draws some conclusions and presents ideas for future work.

- Annex A lists the REST APIs naming practices from ontology elements defined in Chapter 4.

- Annex B provides a template for the API specification introduced in Chapter 4 .

- Annex C presents the questionnaire given to the participants to perform the evaluation in Chapter 5 .

\subsection{Related publications}

During the development of this thesis, different results were accepted in journals, as well as published in international conferences and workshops. Such publications, and other related outcomes, are gathered in this section.

\section{- Journal publications}

- Espinoza-Arias, P., Garijo, D. and Corcho, O.. Crossing the Chasm Between Ontology Engineering and Application Development: A Survey. Journal of Web Semantics. This work presents part of the state of the art study described in Chapter 2 .

- Espinoza-Arias, P., Poveda-Villalón, M., and Corcho, O.. Using LOT methodology to develop a noise pollution ontology: a Spanish use case. This work presents the process followed to develop an ontology as part of the field research work described in Chapter 5 . 
- Espinoza-Arias, P., Fernández-Ruiz, M. J., Morlán-Plo, V., Notivol-Bezares, R., and Corcho, O.. The Zaragoza's Knowledge Graph: Open Data to Harness the City Knowledge. This work presents the analysis of the manual API generation process from ontologies as part of the field work described in Chapter 5 .

\section{- Conference publication}

- Espinoza-Arias, P., Garijo, D. and Corcho, O.. Coming to Terms with FAIR Ontologies. 22nd International Conference on Knowledge Engineering and Knowledge Management, EKAW 2020. Bolzano, Italy, September 16-20, 2020. This work presents the need to publish ontologies following the FAIR principles in order to be compliant with data published under the same principles described in Chapter 4.

\section{- Workshop publication}

- Espinoza-Arias, P., Garijo, D. and Corcho, O.. Mapping the Web Ontology Language to the OpenAPI Specification. International Conference on Conceptual Modeling. This work presents the mapping between OWL and OAS described in Chapter 4 



\section{Chapter 2}

\section{State of the Art}

This chapter presents a summary of the state of the art that is relevant for this thesis. First of all, the chapter starts with Section 2.1 that provides the definitions that will be used in this thesis. Then, Section 2.2 presents the most relevant and well-known methodologies for ontology development with a special focus on the stages and activities they propose. Next, Section 2.3 presents an overview of approaches, techniques, and tools for API generation from ontologies. Finally, Section 2.4 concludes the chapter with a summary of the main conclusions and current limitations in the existing methodologies for ontology development and the approaches for API generation from ontologies, which will help to motivate the contributions of this thesis.

\section{$2.1 \quad$ Terminology}

In this section, the main definitions that will be used throughout this thesis are provided.

- Ontology artefact. Intermediate or final element generated or gathered during the ontology development process. Some artefacts are designated to motivate and assess an ontology such as use cases, user stories, or competency questions. Other artefacts are concerned to the ontology implementation such as conceptualization model, or ontology serialization.

- Competency question. Ontology artefact that allows specifying the knowledge that the ontology should represent and its functionality. A competency question can also be understood as a requirement that the ontology must fulfill, thus it is commonly used to validate the ontology. 
- Glossary of terms. Ontology artefact that is usually generated to extract key terms and definitions from the documents, data, requirements, among other inputs analyzed during the ontology specification activity

- Ontology serialization. Ontology artefact that allows formalizing an conceptualization model of an ontology into a formal language.

- SPARQL query. Ontology artefact that is usually generated to test if data represented with the ontology gives back the answer expected for a competency question. It is defined according to the syntax defined in the SPARQL query language specification 1

- SPARQL endpoint. Point of access on an HTTP network capable of receiving and processing SPARQL queries. Behind this endpoint there is a triple store, compliant with the SPARQL protocol, where RDF data resides.

- API path. Resource that an API exposes, e.g. the API path / countries exposes a set of countries resources.

\subsection{Methodologies for ontology development}

Over the years a series of methods and methodologies have been reported for developing ontologies. This section provides a brief description of the most relevant methodologies in regards to the stages they involve and how they have evolved from representing the ontology in a formal language to its usage in applications.

\subsubsection{Methodology by Grüninger and Fox}

This methodology (Grüninger and Fox, 1995) defines a set of steps aimed at building a model for knowledge representation using first order logic. The resulting model have the ability to deduce answers to queries. The steps proposed in this methodology are depicted in Figure 2.1 and described below.

First, a set of motivating scenarios are proposed. These scenarios describe possible applications which the ontology will be used or story problems that are not properly addressed by existing ontologies. Then, based on the scenarios proposed in the preceding

1 https://www.w3.org/TR/rdf-sparql-query 
step, a set of queries written in natural language and their answers are formulated. These queries are known as informal competency questions and are considered as the requirements that the ontology must be able to answer.

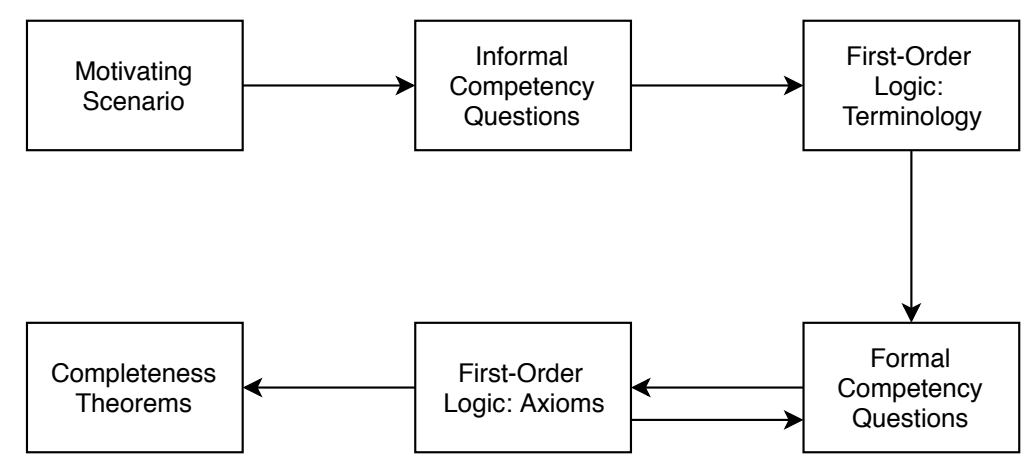

Figure 2.1: Procedure for ontology design and evaluation (Grüninger and Fox, 1995)

Afterwards, a set of terms are specified using first order logic. These terms are the main concepts, attributes, and relationships gathered from the informal competency questions and their answers. Then, the competency questions are defined formally as an entailment or consistency problem in regards to the axioms in the ontology.

Next, axioms are included using first-order-logic in order to specify the definitions of terms and constraints in their interpretation. If these axioms are not enough to represent the formal competency questions, additional axioms must be added in an iterative process until the ontology is sufficient. Finally, the conditions under which the solutions to the questions are complete must be defined. This forms the completeness theorems for the ontology that allow testing whether the ontology satisfies the competency questions.

In summary, this methodology provides a guide to transform informal scenarios into computable models by taking advantage of the classic logic. These scenarios arise in the applications; therefore the methodology is grounded in an strategy for building applications. In addition, it introduces the competency questions as a way of extracting the ontology requirements against which the ontology can be tested. However, the methodology does not provide details of activities or techniques to carry out each step nor how to use the ontology in applications. 


\subsubsection{METHONTOLOGY}

This methodology (Fernández-López et al., 1997) describes how to build ontologies either from scratch, reusing other ontologies as they are, or by a process of reingeniering them. The methodology also identifies an ontology development process which includes a set of activities to be carried out when building ontologies. These activities are based on the main activities identified for the software development process and those of the knowledge engineering methodologies. In addition, the methodology proposes a life cycle which allows identifying the stages that the ontology should pass during its lifetime, and the interpendencies (or relationships) with the life cycle of other ontologies. This life cycle is based on evolving prototypes; therefore, changes can be made to the ontology at each iteration.

Figure 2.2 depicts the ontology development process and life cycle proposed by this methodology. The activities identified in the ontology development process are grouped in management, development, and support activities. It can bee see in this figure that the management and support activities are carried out simultaneously into development activities. In addition, it can be observed that the conceptualization is the activity that more attention requires specially at the knowledge acquisition and evaluation activities.

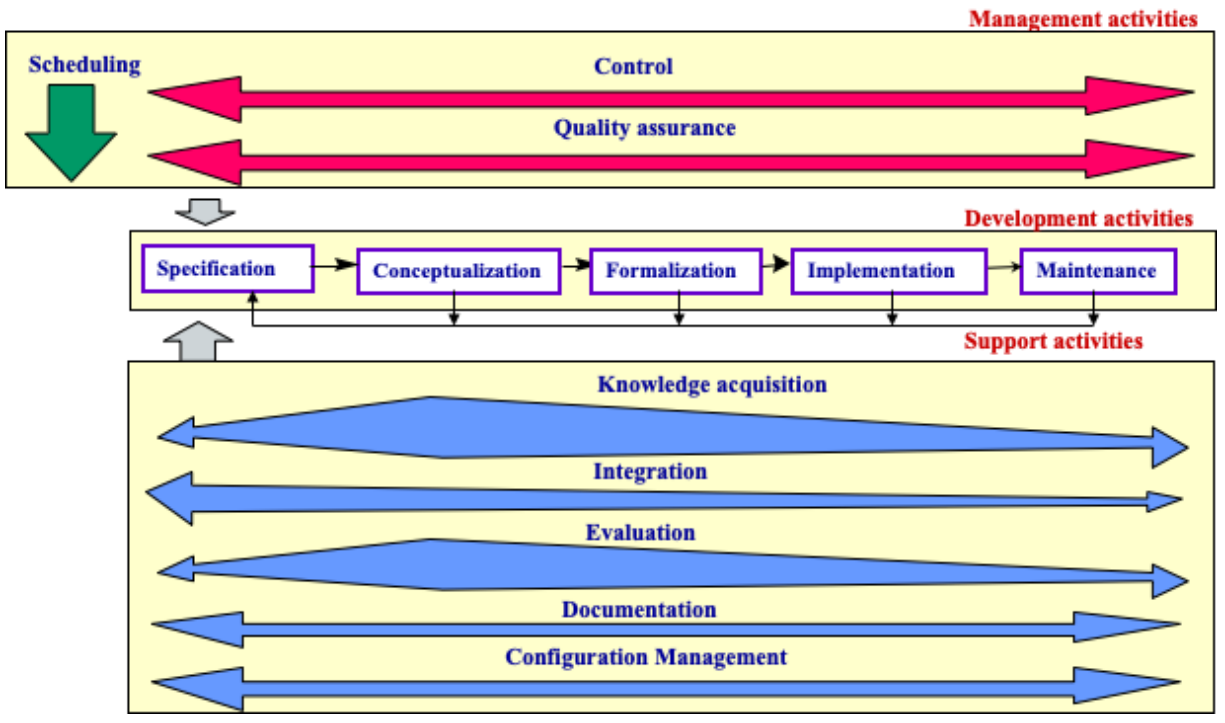

Figure 2.2: Ontology development process and life cycle in METHONTOLOGY (GómezPérez et al., 2006 
In summary, METHONTOLOGY identifies the ontology development process, including the set of activities to be carried out in this process. It also identifies the ontology life cycle; therefore, it allows to determine when and the order of execution of the activities of the development process. The methodology also provides details on the techniques used in each activity, the outputs of each activity, and how to evaluate these outputs. In this methodology, the last activity identified in the development process is the maintenance, which allows updating and correcting the ontology. However, METHONTOLOGY does not include any detail on how to use the ontology in applications.

\subsubsection{On-To-Knowledge}

This methodology (Staab et al. 2001) proposes a set of phases to build applicationdriven ontologies; therefore, it has a strong focus on the future use of the ontology in applications based on usage scenarios. The phases identified in the methodology are depicted in Figure 2.3 and those regarding to the ontology development are described below.

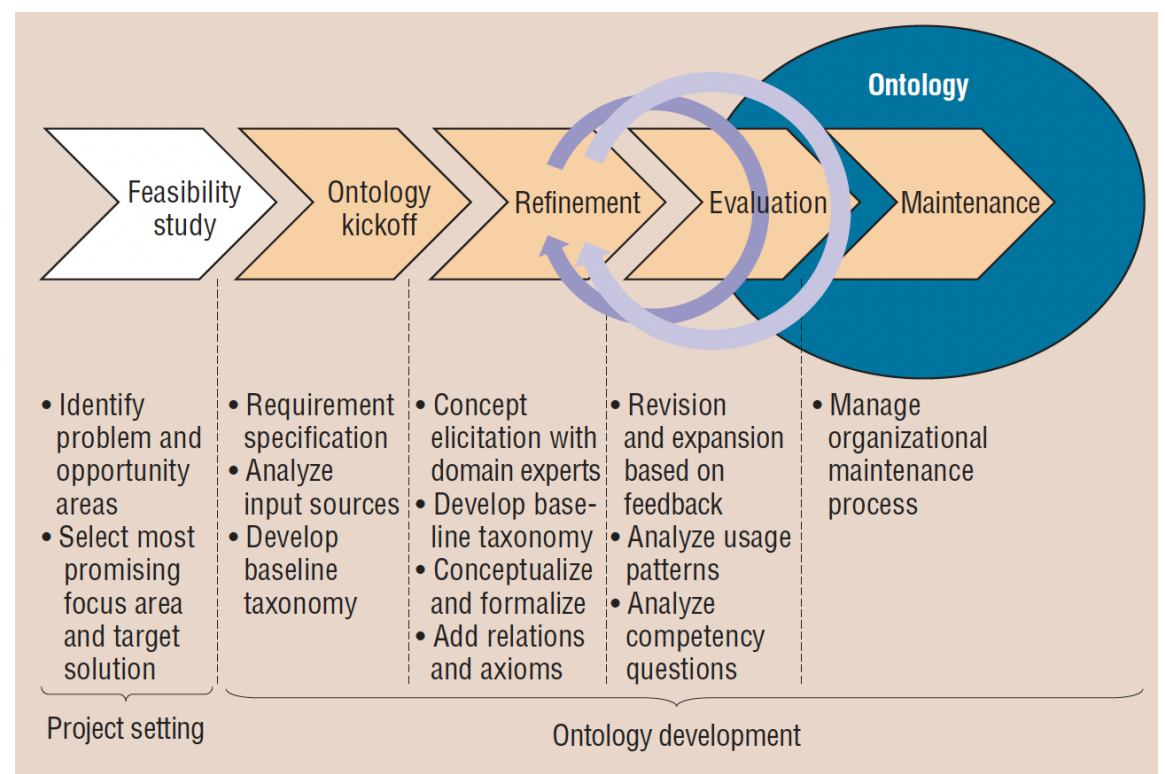

Figure 2.3: On-To-Knowledge development process or knowledge metaprocess (Staab et al. 2001)

In the kickoff phase the ontology requirements are specified, which includes deter- 
mining the ontology's goal, defining its domain and scope, posing competency questions, and identifying potential users and usage scenarios. In this phase potential reusable ontologies are also analyzed and a semi-formal description of the ontology is built. Then, in the refinement phase the initial semi-formal description is polished by means of the interaction with the domain experts, and it is formalized into a mature application-oriented ontology. In the evaluation phase, the ontology is checked to determine whether it answers the competency questions, and the ontology is tested in the target application environment. The evaluation and refinement phases are linked and several iterations between them are needed until get the target ontology. Finally, in the maintenance phase the methodology proposes to perform the ontology maintenance as part of the application.

In summary, On-To-Knowledge proposes an incremental and cyclic ontology development process. Despite this methodology allows building ontologies that are highly dependent on the application, it does not detail how the ontology is used for building such application. Details on the usage of ontology based systems are described by the authors of the methodology, but they are defined as a knowledge process that comes after implementing the system.

\subsubsection{NeOn Methodology}

This methodology (Suárez-Figueroa et al., 2015) provides a set of scenarios for building ontologies and ontology networks. Its main focus is providing guidelines for reusing an reengineering resources (ontological and non-ontological), and to allow collaboration in the ontology development process. The scenarios proposed by the methodology are illustrated in Figure 2.4 and listed below:

- Scenario 1: From specification to implementation.

- Scenario 2: Reusing and reengineering non-ontological resources.

- Scenario 3: Reusing ontological resources.

- Scenario 4: Reusing and reengineering ontological resources.

- Scenario 5: Reusing and merging ontological resources.

- Scenario 6: Reusing, merging and reengineering ontological resources. 
- Scenario 7: Reusing Ontology Design Patterns.

- Scenario 8: Restructuring ontological resources.

- Scenario 9: Localizing ontological resources.

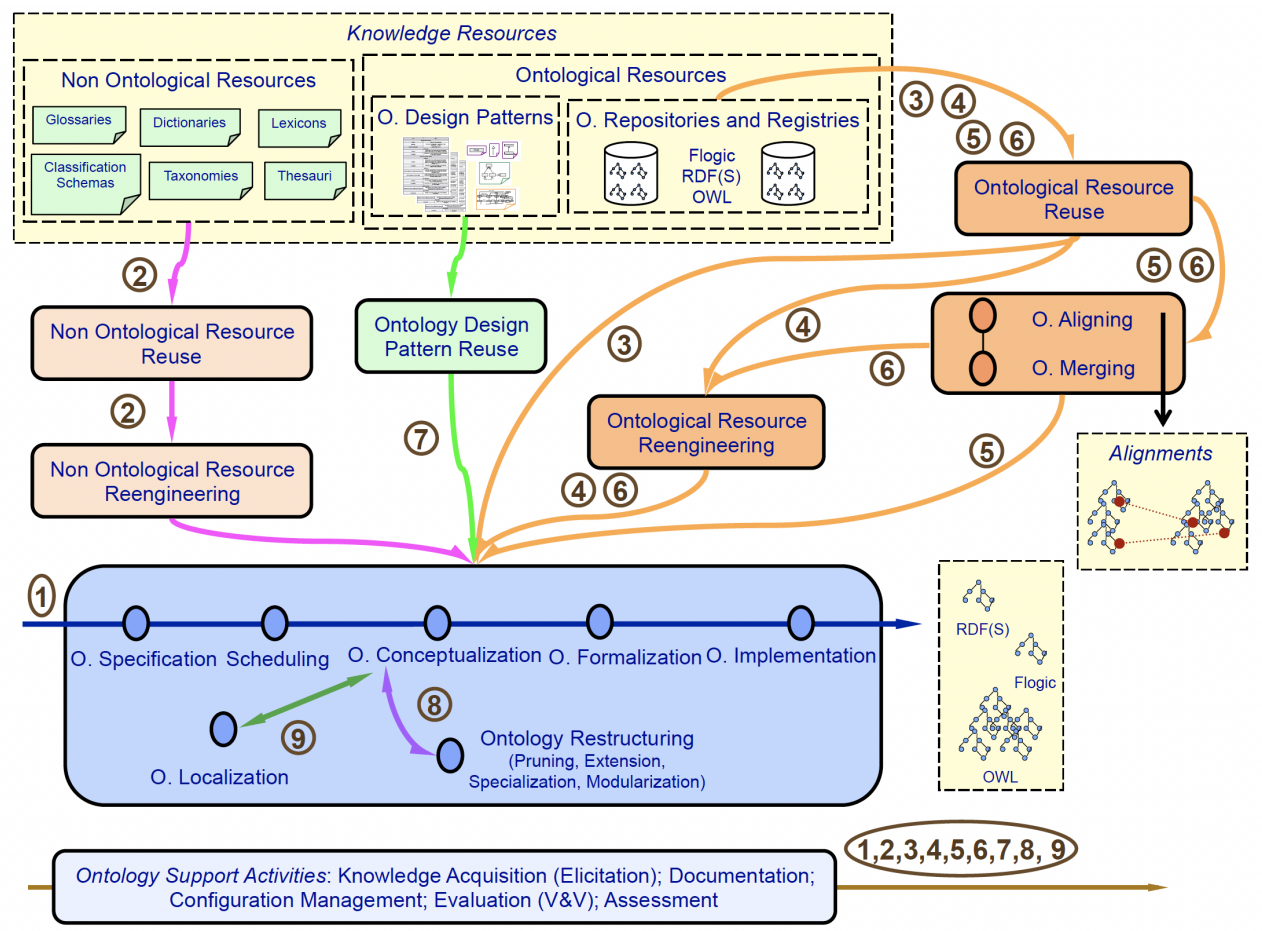

Figure 2.4: NeOn scenarios for building ontology networks (Suárez-Figueroa et al. 2015)

Scenario 1 is the most common scenario in any development process; therefore, it is mandatory to always use it or combine it with the other scenarios if required. The set of activities depicted at the bottom of Figure 2.4 (elicitation, documentation, configuration management, evaluation, and assessment) should be carried out during the development process.

In summary, NeOn is a scenario-based and collaborative methodology for building ontologies and ontology networks. It provides detailed guidelines for the scheduling and ontology requirement specification activities, and the ontological resources reuse process. In addition, it proposes two ontology life cycle models to organize ontology developments and a glossary of processes and activities to unify the ontology engineering terminology. 
However, this glossary does not include a definition for the ontology exploitation/usage activity nor does methodology include any details on how to use the ontology.

\subsubsection{SAMOD}

The SAMOD (Simplified Agile Methodology for Ontology Development) (Peroni, 2016) is an agile methodology for ontology development by means of an iterative workflow that focuses on creating well-developed and documented models starting from exemplar domain descriptions. Figure 2.5 shows a summary of the iterative steps proposed in the methodology.

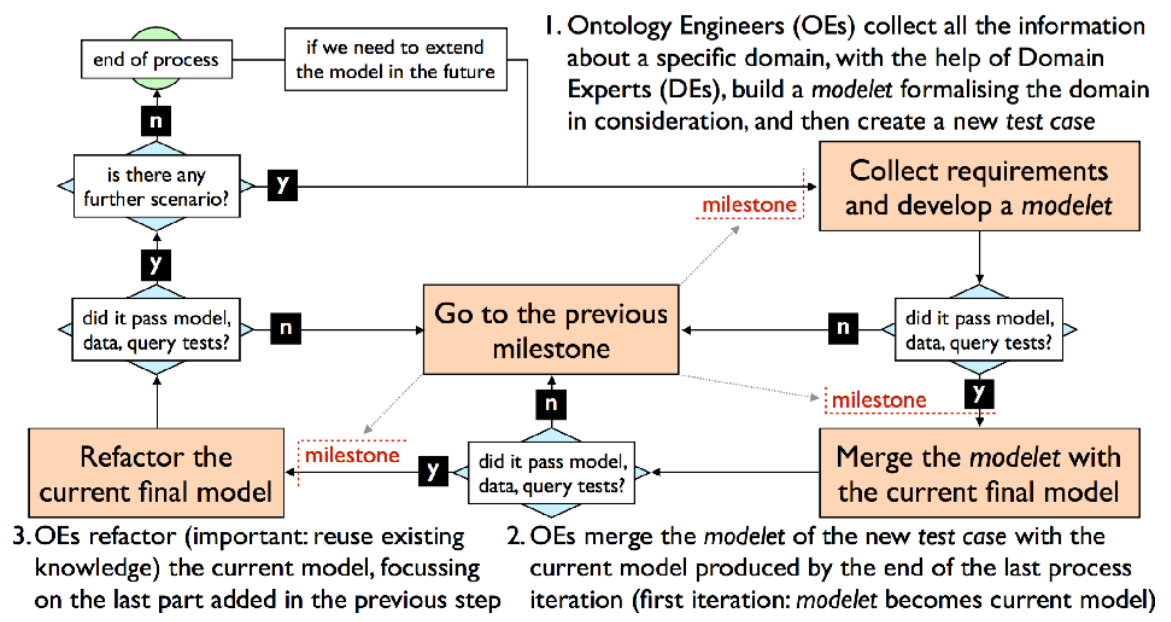

Figure 2.5: A brief summary of SAMOD, starting with the "Collect requirements and develop a modeled" step (Peroni, 2016)

The first step regards to define a new test case. To do so, the ontology engineers and domain experts define a motivating scenario. Then, they propose a set of informal competency questions. Next, they define a glossary of terms based on the scenario and the competency questions. From them, the ontology engineer develops a modelet, which is a model that describes a particular aspect of the domain. Then, an exemplar dataset is generated to formalize the examples introduced in the motivating scenario according to the modeled. After that, a SPARQL query is generated for each competency question.

The second step regards to merge the current model with the modelet. To this end, the ontology engineers should merge the modelet with the current model, i.e. the version of the final model released in the last iteration. Finally, the third step refers to refactor the current model. To do that, the ontology engineer refactors the current model by 
reusing existing knowledge, documenting it, and enriching the current model by using the capabilities offered by OWL2.

At the end of an iteration each test case is stored for documentation that could be useful for end-users. Each test case provides natural language descriptions (the motivating scenario, competency questions and glossary of terms) of a particular aspect of the domain described by each model. It also includes the exemplar dataset and the set of SPARQL queries defined.

In summary, SAMOD is an agile methodology that defines an iterative and incremental ontology development process. The methodology proposes a set of steps for building the ontology that allows releasing ontologies that are always ready-to-use and easily-understandable by humans. The method relies on the definition of test cases that allows integrating in each iteration a specific aspect of the domain. However, SAMOD does not provide details on how to maintain and use the ontology.

\subsubsection{LOT}

The LOT (Linked Open Terms) methodology (Poveda-Villalón et al., 2019) is based on agile techniques in which sprints and iterations represent the main workflow organization to align the ontology development with software development agile practices. It is build on top of the previous activities defined in the NeOn methodology. Figure 2.6 shows the base workflow proposed in LOT ${ }^{2}$ and its main activities are described below.

In the ontology requirements specification activity the goal and the scope of the ontology are defined. In addition, the set of requirements that the ontology should fulfill are identified and defined in form of competency questions. Then, in the implementation activity the ontology is built based on the requirements and using a formal ontology implementation language (e.g. OWL). This activity is carried out in different sprints in which a set of requirements are selected to be implemented and after each iteration a new version of the ontology is produced.

Next, in the publication activity the ontology is made available on-line both as human-readable documentation and in a machine-readable format (which is obtained from the implementation activity). Finally, in the maintenance activity the ontology can be updated after the last release to, for example, include new requirements, fix bugs

2 https://lot.linkeddata.es 


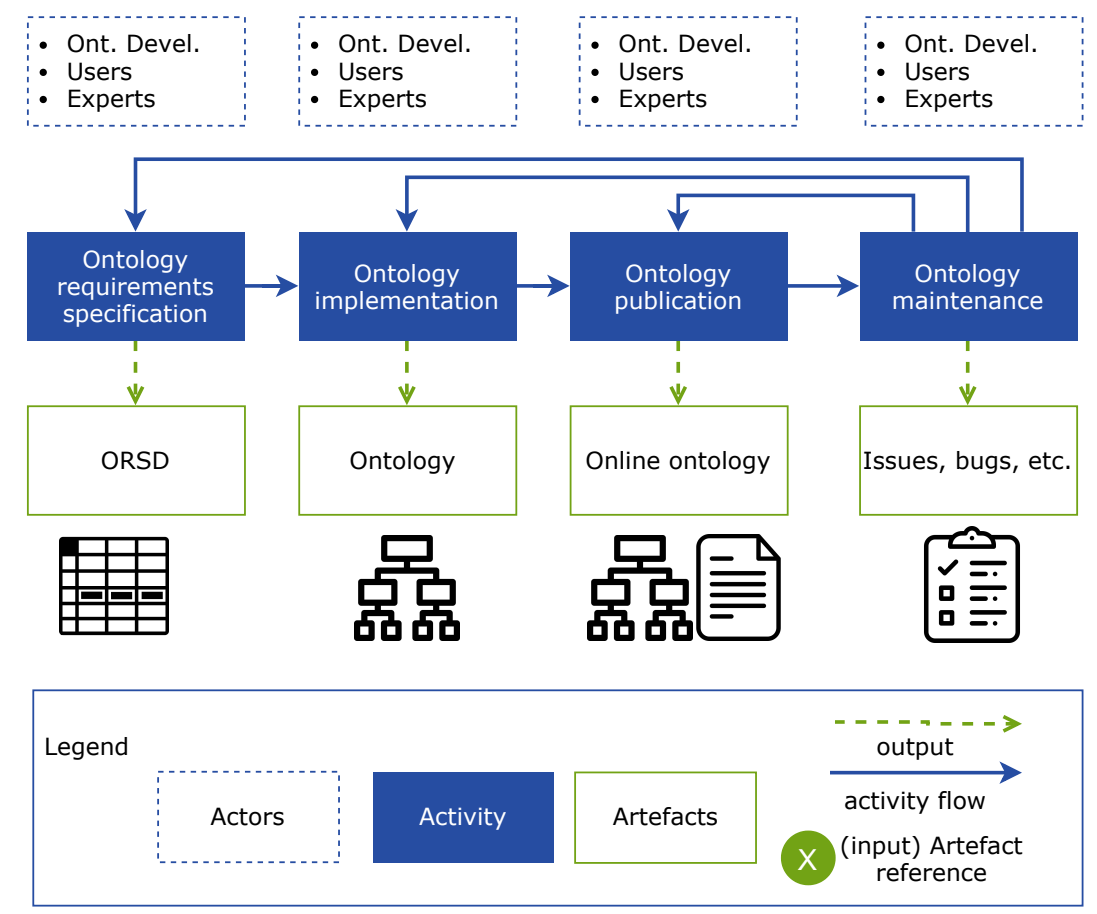

Figure 2.6: LOT methodology base workflow of processes (Poveda-Villalón et al. 2019$)$

found in the ontology, cover improvement suggestions, etc., which in consequence may derive in another ontology development iteration.

In summary, LOT proposes a lightweight and agile methodology for ontology development. It defines a set of activities and details how they should be carried out, some of these details are reused from the NeOn methodology. In addition, the methodology identifies the main actors that conforms the ontology development team and describes a set of artefacts that are produced at each activity. This methodology proposes the publication activity that is not defined or mentioned in previous methodologies. However, LOT does not describe how to use the ontology after its publication.

\subsection{Approaches for API generation from ontologies}

This section presents the findings obtained from the systematic literature review we conducted, as it is reported in (Espinoza-Arias et al., 2021). In such review, our main objective was to find, analyze, and compare existing API-based applications and tools for making KG data more accessible to application developers, and helping ontology 
engineers guide application developers in $\mathrm{KG}$ data consumption. The findings obtained from the review were categorized into specifications, and into tools and technologies. Section 2.3.1 describes the main specifications that are followed to define the APIs. Section 2.3 .2 presents the technologies and tools that enable the consumption of KG data through APIs. Finally, Section 2.3 .3 provides an analysis of the specifications, technologies and tools previously described.

\subsubsection{Specifications}

There are several specifications to describe the design and details on how to implement APIs. The subsections 2.3.1.1 to 2.3.1.7 presents a summary of those specifications that have been defined in the Semantic Web community. Then, subsections 2.3.1.8 and 2.3.1.9 presents the specifications defined by the Software Development community and that have been reused in solutions proposed by the Semantic Web community.

\subsubsection{SPARQL Protocol}

The SPARQL Protocol and RDF Query Language (Clark et al. 2008) describes the means for conveying SPARQL queries and updates to a SPARQL processing service and returning the results via HTTP to the entity that requested them. It was the first standard to provide access to RDF data. Therefore, most of the projects that had published RDF data use this protocol through a server implementation. The latest version is the SPARQL 1.1 Protocol (Feigenbaum et al., 2013).

\subsubsection{SPARQL 1.1 Graph Store Protocol (GSP)}

Protocol that describes HTTP operations for managing a collection of RDF graphs from a SPARQL triplestore (Ogbuji, 2013). To this end, GSP describes a mapping between HTTP methods and SPARQL queries. This protocol can be viewed as a lightweight alternative to the SPARQL 1.1 protocol in combination with the full SPARQL 1.1 Query and SPARQL 1.1 Update languages.

\subsubsection{Linked Data API (LDA)}

Specification that defines a configurable API layer intended to support the creation of simple RESTful APIs over RDF triplestores (Google, 2010a). This configuration must 
be provided by means of an RDF file that follows a specific vocabulary and processing model to describe the SPARQL endpoint, variables, pagination, queries and all the details needed for the API generation.

\subsubsection{Hydra Vocabulary}

Lightweight vocabulary designed to create hypermedia-driven Web APIs (Lanthaler and Gütl, 2013). Hydra defines a set of common concepts to create generic APIs; enabling servers to advertise valid state transitions to a client. Clients can use this information to construct HTTP requests to achieve a goal by modifying the state of the server.

\subsubsection{Linked Data Platform (LDP)}

Specification that defines a set of rules for HTTP operations on web resources to provide an architecture for read-write Linked Data on the Web (Arwe et al., 2015). LDP provides details on how to configure HTTP access to manage resources (HTTP resources) and containers (collections of resources). Resources can be RDF sources and non-RDF sources (e.g. binary or text documents). Containers are defined only for RDF resources and they can be Basic, Direct, and Indirect. Basic containers contain triples of arbitrary resources, and must be described by a fixed structure using and a specific vocabulary ${ }^{3}$ Direct containers specialize Basic containers by introducing membership triples which allows the subject and predicate of the triple to be configured using the container definition. Indirect containers are similar to Direct containers but they also are capable of having members whose objects have any URI.

\subsubsection{Linked Data Templates (LDT)}

Protocol that specifies how to read-write Linked Data based on operations backed by SPARQL 1.1 (Jusevičius, 2016). LDT defines an ontology with the core concepts and properties required to describe applications. The ontology must be reused to design application ontologies that contain API paths, operations, SPARQL queries, and state change instructions for the desired application. State changes intend to cover the hypermedia definition provided in the REST architecture (Fielding and Taylor, 2002), which states that web resources should specify their next state.

$\sqrt[3]{\text { https: / /www.w3.org/ns/ldp\# }}$ 


\subsubsection{Social Linked Data specification (Solid)}

Specification (Berners-Lee et al., 2019) that describes implementation guidelines for servers and client applications to enable decoupling data from services. Solid provides support for a decentralized Web where users can store their personal data on Solidcompliant servers and choose which applications can access such data. Likewise, Solidcompliant applications allow managing any user's data stored on the aforementioned servers. This specification extends the Linked Data Platform to provide a REST API for read and write operations on resources and containers. Solid also provides a WebSocketbased API with a publish/subscribe mechanism to notify clients of changes affecting a given source in real time.

\subsubsection{OpenAPI Specification (OAS)}

Formerly known as the Swagger Specification, OAS (Initiative, 2011) defines how to describe REST APIs in a programming language-agnostic interface in order to allow humans and machines to discover and understand the details of a service. OAS has become the choice of reference by many developers due to its community support and the amount of available tools for creating API documentation, server and client generation and testing.

\subsubsection{GraphQL}

Specification (GraphQL, Foundation, 2015) that uses a declarative query language to allow clients accessing the data they need on demand. In GraphQL, queries define the available entry points for querying a GraphQL service. GraphQL has become popular among the developer community as an alternative to REST-based interfaces, as it presents a flexible model rather than a static API. However, developers must be familiar with the schema used to represent the queried data.

\subsubsection{Technologies and Tools}

In the Semantic Web community, several technologies and tools for generating APIs to enable KG consumption have been proposed. In the following subsections, we present a brief description of each of those found in the literature review. 


\subsubsection{KG stores}

Several graph databases (e.g. Neo4j (Miller, 2013)) and triplestores (e.g. Fuseki (Apache, 2014), Blazegraph (SYSTAP, 2015), GraphDB (Ontotext, 2015) ) can be used for KG storage. As a representative example (resulting from our literature review) we include OpenLink Virtuoso (Erling and Mikhailov, 2009), a hybrid data store and application server supporting the SPARQL 1.1 Protocol that has been widely used in the Semantic Web community. Virtuoso can be configured as an implementation backend on some of the specifications presented in the previous section, e.g., as a Linked Data Platform client and server.

\subsubsection{Pubby}

Linked Data compliant server that adds a simple HTML interface and dereferenceable URIs on top of SPARQL endpoints (Cyganiak and Bizer, 2007). Thanks to Pubby, users can navigate the contents of an endpoint interactively in their browser, without having to issue any SPARQL queries. Pubby handles content negotiation and includes an extension to describe the provenance of each request made to the server (Hartig et al. 2010).

\subsubsection{Puelia}

PHP implementation of the Linked Data API (Google, 2010b). Puelia allows handling incoming requests by reading a configuration file and executing the corresponding SPARQL queries defined in such file. The RDF data retrieved from the SPARQL endpoint is returned to the client in several formats (e.g. Turtle, JSON, etc.).

\subsubsection{Epimorphics Linked Data API Implementation (ELDA)}

Java implementation of the Linked Data API (Epimorphics, 2011). ELDA provides a way to create APIs to access RDF data using RESTful URLs; as well as a mechanism to create resource-specific views for browsing these data. As with Puelia, in ELDA all URLs are translated into SPARQL queries to get data from a target SPARQL endpoint. 


\subsubsection{Linked Open Data Inspector (LODI)}

Linked Data server that provides HTML views and content negotiation of resources on a SPARQL endpoint (Fernández-Sellers, 2015). LODI was inspired by Pubby, but it includes extra functionalities such as more detailed and customizable views for developers, map-based location graphs in case resources contain geospatial attributes, automatic detection and display of image files, and custom configuration for host portal information.

\subsubsection{Apache Marmotta}

Linked Data server (Foundation, 2013) compliant with the SPARQL Protocol 1.1 (providing a SPARQL endpoint). Marmotta was one of the first tools which implemented the Linked Data Platform specification, with support for LDP Basic Containers and content negotiation. Moreover, Marmotta is a Linked Data development environment which includes several modules and libraries for building Linked Data applications.

\subsubsection{Building Apis SImpLy (BASIL)}

Framework designed for building Web APIs on top of SPARQL endpoints (Daga et al. 2015). In BASIL, a set of SPARQL queries and their related endpoints must be defined. In addition, API parameters can be included according to a SPARQL variable naming convention. This convention allows using parameters in configurable templates to parametrize SPARQL as an API. Then, BASIL generates the API paths to retrieve the data and the Swagger specification documentation of the API.

\subsubsection{Git repository linked data API constructor (GRLC)}

Server implementation that takes SPARQL queries and translates them to Linked Data Web APIs (Meroño-Peñuela and Hoekstra, 2016). These queries can be stored in GitHub repositories, local filesystem, or listed as online available URLs into a YAML file. In addition, these queries must include SPARQL decorators $4^{4}$ (or tags) to add metadata and comments, e.g. to define the specific HTTP method to be executed, the query-specific endpoint, pagination, among others. Then, GRLC takes each query and translates it into one API operation and generates a JSON Swagger-compliant specification and

$\sqrt[4]{\text { https://github.com/CLARIAH/grlc/tree/dev\#decorator-syntax }}$ 
a Swagger-UI to provide the interactive API documentation. In addition, GRLC has recently included a mechanism (provided by SPARQL Transformer (Lisena et al., 2019)) to translate a JSON structure, defined according to specific rules, into a SPARQL query. This mechanism allows transforming SPARQL query results into a JSON serialization.

\subsubsection{AtomGraph Procesor}

Linked Data processor and server for SPARQL endpoints (Jusevičius, 2016) (earlier known as Graphity (Jusevicius, 2014)). AtomGraph uses an ontology for HTTP request matching and response building. This ontology contains Linked Data Templates that map URI templates to the SPARQL queries needed to request matching and response building. The SPARQL queries are included into the application ontology using the SPIN-SPARQL Syntax model $5^{5}$

\subsubsection{JSON-QB API}

Interface for developers that reuses statistical data stored as RDF Data cubes Zeginis et al., 2017) (Stasiewicz and Waqar, 2016). JSON-QB only works for data represented with the W3C RDF Data Cube vocabulary, and has evolved into CubiQL ${ }^{6}$ a GraphQL service for querying multidimensional Linked Data Cubes.

\subsubsection{Open Semantic Framework (OSF)}

Framework designed to create and manage domain specific ontologies; and to maintain, curate and access the stored data (Mayer et al., 2017). Data access is enabled through a REST API based on prefabricated SPARQL query templates.

\subsubsection{Trellis}

Linked Data server which supports high scalability, large quantities of data, data redundancy and high server loads (Jansen et al., 2019). Trellis follows the Linked Data Platform specification for resource management and has several extensions 7 implement-

\footnotetext{
$\sqrt[5]{\text { https://spinrdf.org/sp.html }}$

6 https://github.com/Swirrl/cubiql

https://github.com/trellis-ldp/trellis-extensions
} 
ing persistence layers and service components e.g. Trellis-Cassandra for distributed storage. Trellis has been included in the Solid Project ${ }^{8}$ Test Suite 9

\subsubsection{Ontology-Based APIs (OBA)}

Framework designed to generate an OpenAPI specification from an ontology or ontology network (specified in OWL) (Garijo and Osorio, 2020). Once a target OpenAPI specification is generated, OBA also provides the means to create a REST API server to handle requests, deliver the resulting data in JSON format (following the ontology structure) and validate the API against an existing KG. OBA automatically generates SPARQL templates for common operations from the source ontology; but also accepts custom queries needed by users. Custom queries are specified following the conventions established by GRLC and Basil.

\subsubsection{RESTful-API for RDF data (R4R)}

Template-based framework that creates RESTful APIs over SPARQL endpoints using customized queries (Badenes-Olmedo et al., 2021). R4R is both a server and a working environment: once started, R4R runs a web service that can be updated when new resources are added without having to restart the server. The workspace in R4R defines all available resources to its service, and contains the SPARQL queries and the templates required for managing input queries and resources obtained from a target endpoint.

\subsubsection{OWL2OAS}

Converter designed for translating OWL ontologies into OpenAPI Specification documents (Hammar, 2020). This tool generates API paths for the concepts of the ontology and their schemas. In addition, OWL2OAS provides JSON-LD context for the aforementioned schemas which is based on the object and data properties defined in the ontology.

\subsubsection{Ontology2GraphQL}

Web application that generates a GraphQL schema and its corresponding GraphQL service from a given RDF Ontology (Farré et al. 2019$)$. To this end, the ontology for

8 https://solidproject.org/

sttps://github.com/solid/test-suite 
data representation must be manually annotated with a GraphQL Metamodel (GQL), which includes several classes representing the GraphQL types that compose a GraphQL schema (e.g. object, list, enumeration, among others). Therefore, each ontology class is mapped to an instance of GQL Object class. Object and datatype properties are defined as instances of GQL ObjectField and ScalarField classes respectively. Finally, there are several GQL properties required to specify more details on properties, for example, the GQL hasModifier property can be used to define that an object property will manage an array of the elements.

\subsubsection{Restful API Manager Over SPARQL Endpoints (RAMOSE)}

Framework designed to create REST APIs over SPARQL endpoints through the creation of textual configuration files (Daquino et al., 2020). Such files enable querying SPARQL endpoints via Web RESTful API calls that return either JSON or CSV-formatted data. To provide this configuration, a hash-format syntax 10 based on a simplified version of Markdown is required.

\subsubsection{Community Solid Server}

Server implementation of the Solid specifications (Van Herwegen et al., 2020). It aims to provide support for data pods, which allows storing personal data in an accessible manner. Solid makes it possible to decouple personal data storage from services, and therefore users are free to decide which applications can access to their pods. As a result, users can keep total control of their data.

\subsubsection{Walder}

Framework that allows configuring a website or Web API on top of Knowledge Graphs (e.g. SPARQL endpoint, Solid pod, etc.) (Heyvaert et al., 2020). To this end, users must define a configuration file with the details of the data source, paths, operations, etc. allowed for the API. Walder reuses the Comunica framework (Taelman et al. 2018a), more precisely the graphql-ld-comunica engine ${ }^{11}$ to execute the queries needed to get the required data. Walder uses GraphQL-LD (Taelman et al., 2018c), a query language which allows extending GraphQL queries with a JSON-LD context. Comunica

\footnotetext{
10 https://github.com/opencitations/ramose\#Hashformat-configuration-file

11 https://github.com/rubensworks/graphql-ld-comunica.js
} 
then takes the GraphQL queries and translates them, based on the JSON-LD context, into SPARQL queries to retrieve the desired data.

\subsubsection{Analysis of specifications, technologies and tools for API defi- nition and generation}

In this section we introduce the frameworks designed to perform a systematic comparison of the specifications, technologies and tools described in Section 2.3. We also discuss the results obtained when applying these frameworks to compare the specifications, technologies and tools.

\subsubsection{Criteria for comparing API specifications}

Table 2.1 summarizes the set of criteria defined in the framework to compare the existing specifications. We are interested in the year when specifications were created in order to understand their evolution over time. We want to know if specifications are officially recognized by an authority (i.e., whether they are a standard or not) or if they have just been adopted by a community without going through a standardization process. We also consider relevant the endpoints supported by specifications, since this allows detecting the different KG data sources (e.g. RDF data dump, SPARQL endpoint, among others). We also consider configuration formats, as they give us an idea of details needed to implement a target specification.

In addition, we evaluate if specifications support configurable queries, which indicate the degree of freedom offered by a specification to manage specific data needs of an application. We analyze the file formats (media types) that developers must expect when using such specification, and whether specifications consider developer-friendly formats or not. We assess the operations for resource management provided by a specification, i.e., create, read, update, delete support $\left(\mathrm{CRUD}^{12}\right)$. We also take into account the authentication techniques proposed by the specifications, since it determines how the specification manages the access to the API methods.

We consider the versioning support to understand whether a specification manages KGs that evolve over time, both in terms of their contents and schema (ontology). We analyze the status codes, since they provide information of the methods suggested by

\footnotetext{
${ }^{12}$ In this work, CRUD is understood as the HTTP POST, GET, PUT, and DELETE operations.
} 
Table 2.1: Comparison criteria for specifications

\begin{tabular}{|l|l|}
\hline Criteria & Description \\
\hline Year & Year the specification was made available \\
\hline Standard & Specification recognition type \\
\hline Endpoint & $\begin{array}{l}\text { Type of point where resources are available and } \\
\text { requests are submitted }\end{array}$ \\
\hline $\begin{array}{l}\text { Configuration } \\
\text { format }\end{array}$ & $\begin{array}{l}\text { The file format to be provided with the details of } \\
\text { the specification }\end{array}$ \\
\hline $\begin{array}{l}\text { Configurable } \\
\text { queries }\end{array}$ & Support for customized queries \\
\hline Media types & File formats supported for data interchange \\
\hline Operations & Allowed methods for managing resources \\
\hline Authentication & $\begin{array}{l}\text { Allowed methods to verify the identity of a user } \\
\text { or process }\end{array}$ \\
\hline Versioning & Support for version management \\
\hline Status codes & Type of response messages to a client's request \\
\hline Resources & $\begin{array}{l}\text { Type of resources allowed such as single, collec- } \\
\text { tion, or nested resources }\end{array}$ \\
\hline Reference & The provenance of the specification details. \\
\hline
\end{tabular}

the specification in order to properly provide details to clients about the execution of API calls. We examine the resources supported by the specification, as it allows us to understand if the specification manages single resource, a collection of resources, or nested resources (i.e., if a resource contains a subcollection of resources). Finally, reference provides information about the origin of the specification details provided in this comparison for provenance purposes.

\subsubsection{API specification comparison}

Table 2.2 shows the comparison between the specifications according to each criterion defined in subsection 2.3.3.1. The symbol "-" indicates that the criterion is not described or detailed in the specification source.

Specifications began to appear in the year 2008, when the SPARQL protocol 1.0 (Torres et al. 2008) was defined; and span until 2019, when the latest Solid specification draft has been made available. Three of the analyzed specifications are W3C Recommendations (SPARQL 1.1 Protocol, Graph Store Protocol, and Linked Data Platform) and three are not recommendations but were defined in W3C Community groups: 
Linked Data Templates by the Declarative Linked Data Apps group 13 Hydra by the Hydra group 14 and Solid by the Solid Community group 15 OpenAPI and GraphQL are now considered de facto specifications, as despite not being officially recognized by a standardization body, they are widely used by the developer community (Postman, $2020)$.

Most of the analyzed specifications from the Semantic Web community support SPARQL endpoints. Solid goes one step further, aiming to support any RDF data source such as RDF data dumps and Linked Data documents in addition to SPARQL endpoints. OpenAPI and GraphQL allows specifying any given endpoint, leaving to the implementations the support for query languages. Specification settings are usually provided in different configuration formats. LDA and LDT must be configured in an RDF file which contains the URI templates required for the API and the required SPARQL queries. OpenAPI and GraphQL are configured in JSON, a developer-friendly format (Severance, 2012), (Safris, 2019), but OpenAPI also supports YAML. In summary, the OpenAPI configuration file must contain the schemas and the API paths that will be implemented, and the GraphQL configuration must define the GraphQL schemas which describes the data sources and the GraphQL queries that define the available entry points for querying a GraphQL service. Finally, Hydra requires a JSON-LD (Kellogg et al., 2014) configuration file. Such format aims to represent Linked Data as JSON with minimal changes, thus it intends to be an alternative for developers interested in semantic data. The Hydra configuration file must contain several details of the API such as the URL of the endpoint, supported schemas, allowed operations, among others.

All specifications, depending on the operations and resources supported, allow configurable queries. For LDA, LDP, and LDT, such queries must be written in the SPARQL query language. GraphQL requires queries written in the GraphQL query language; and OpenAPI supports multiple languages, e.g. SQL, since it doesn't restrict the type of endpoint. As for query execution, the specifications provide data results in several media types. SPARQL 1.1 Graph Store Protocol, Hydra, and LDT specifications only provide results in RDF formats (e.g. Turtle, JSON-LD, etc.), while the remaining specifications also support non-RDF formats (e.g. HTML, CSV, JSON, among others).

\footnotetext{
$\sqrt[13]{\text { https: //www.w3.org/community/declarative-apps/ }}$

${ }^{14}$ https://www.w3.org/community/hydra/

15 https://www.w3.org/community/solid/
} 


\begin{tabular}{|c|c|c|c|c|c|c|c|c|c|}
\hline 遏 & 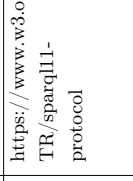 & 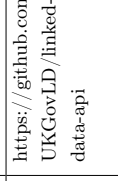 & 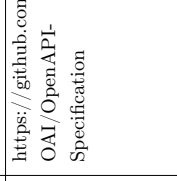 & 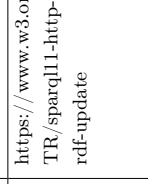 & 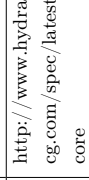 & 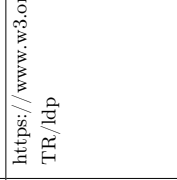 & 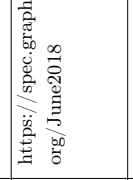 & 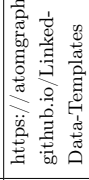 & 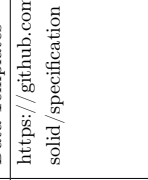 \\
\hline 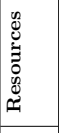 & 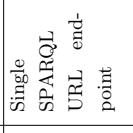 & 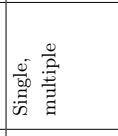 & 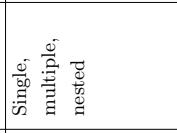 & 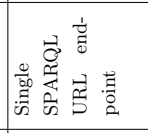 & 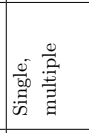 & 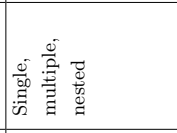 & 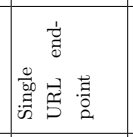 & 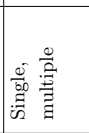 & 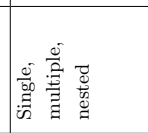 \\
\hline 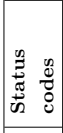 & 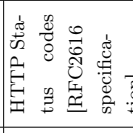 & & 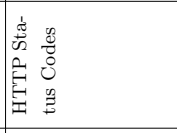 & 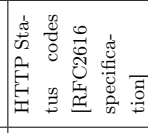 & 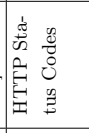 & 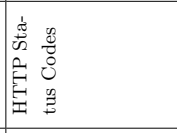 &. & 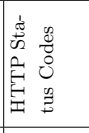 & 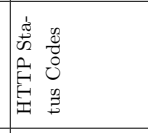 \\
\hline 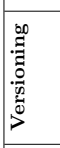 & 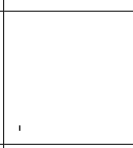 & ' & $\mathscr{s}$ & t & . & . & 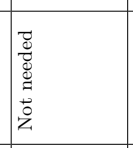 & $t_{-1}$ & . \\
\hline 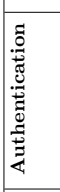 & 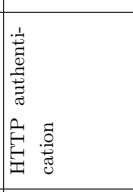 & $\mathrm{d}=\mathrm{s}-\mathrm{s}$ & 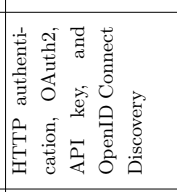 & 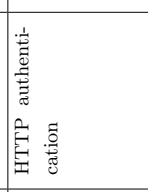 & i & 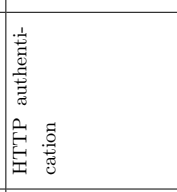 & 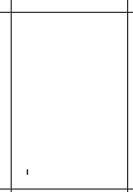 & & 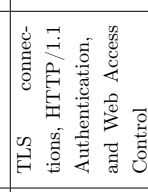 \\
\hline 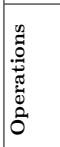 & 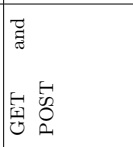 & 葸 & 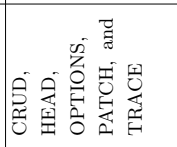 & 層 & 递 & 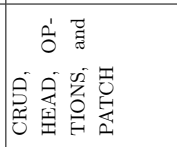 & 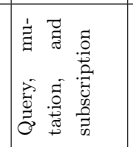 & 尊 & 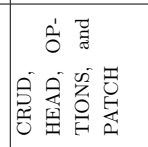 \\
\hline 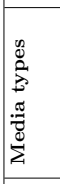 & 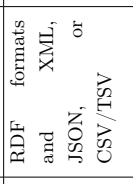 & 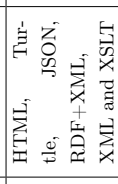 & 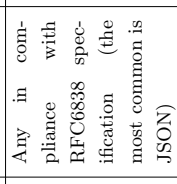 & 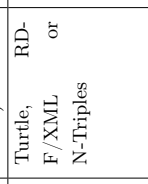 & 言 & 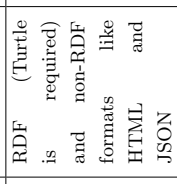 & 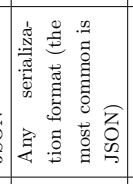 & 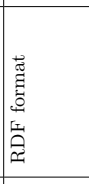 & 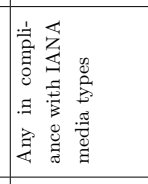 \\
\hline 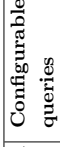 & & $\mathscr{B}$ & $\mathscr{0}$ & 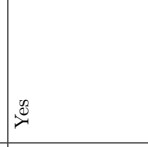 &. & $\mathscr{B}$ & 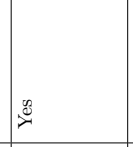 & 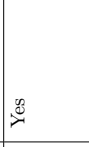 & 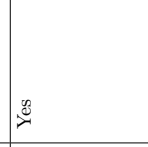 \\
\hline 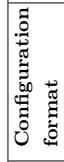 & & 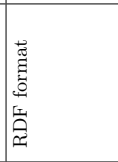 & 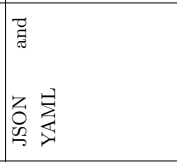 & & 言 & , & 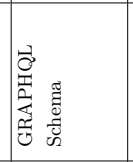 & 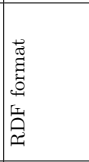 & \\
\hline 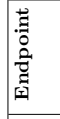 & 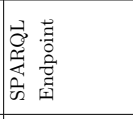 & 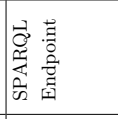 & 安 & 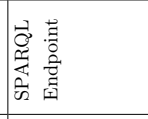 & . & 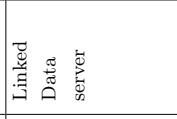 & 宣 & 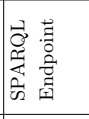 & 嵅 \\
\hline 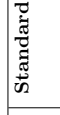 & 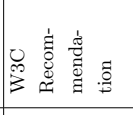 & 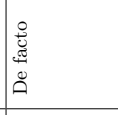 & 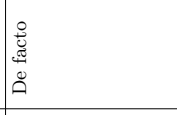 & 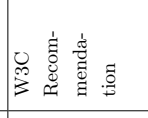 & $\AA$ & 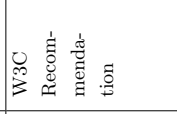 & 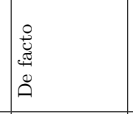 & $\approx$ & 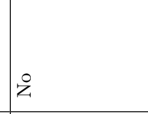 \\
\hline 苟 & 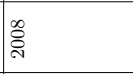 & 高 & जे & :ึّ & ڤั) & 离 & 商 & तें & क्षे \\
\hline 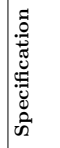 & 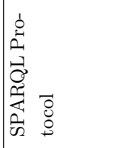 & 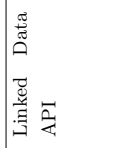 & 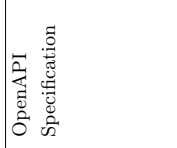 & 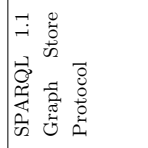 & 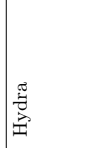 & 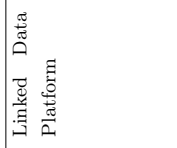 & 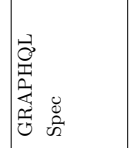 & 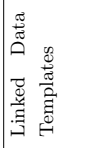 & 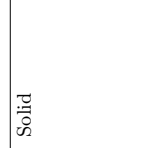 \\
\hline
\end{tabular}


Regarding allowed operations, the most limited specification is Linked Data API since it only supports reading data (GET). The SPARQL Protocol initially supported GET and POST operations, but after the SPARQL 1.1 Graph Store Protocol was introduced, its support was expanded to full CRUD. Hydra and Linked Data Templates support the configuration of CRUD methods, while Linked Data Platform, Solid and OpenAPI, in addition to CRUD, also support HEAD (to ask for information about resources), OPTIONS (to describe the communication options of a resource), and PATCH (to partially update resources) methods. Moreover, OpenAPI supports the TRACE method, which allows to follow the path that a HTTP request follows to the server and it is generally used for diagnostic purposes. GraphQL supports operations with different names but that may be equated to HTTP methods: query implements a GET, mutation implements a POST, and subscription implements a PUT, PATCH, or DELETE.

Most specifications support HTTP authentication. Solid also allows TLS connections for data pods through the https URI scheme, HTTP/1.1 authentication and it must conform to the Web Access Control specification. Solid clients must support HTTP/1.1 Authentication. OpenAPI allows configuring other authentication mechanisms like API key, OAuth2, among others. In order to control the changes in the API, only OpenAPI provides an specific attribute to define the versioning, by following a Semantic Versioning 2.0.0 ${ }^{16}$ convention. In GraphQL it is not needed to specify versions since the specification strongly encourages the provision of tools to allow for the evolution of APIs. To this end, GraphQL tools must allow API providers to specify that a given type, field, or input field has been deprecated and will disappear at some point in time; thus they must notify clients by, for example, message responses detailing on changes. Therefore, GraphQL systems may allow for the execution of requests which at some point were known to be free of any validation errors, and have not changed since. The remaining specifications leave versioning up to the implementations.

The OpenAPI, LDP, and Solid specifications support single, collection, and nested resources since they led implementers to freely define them. LDA, Hydra and LDT support single and collection of resources only. In the case of the SPARQL Protocol and SPARQL 1.1 Graph Store Protocol both support a single URL referring to the SPARQL endpoint. In a similar manner, GraphQL only requires a single URL. Finally, almost all specifications support reusing the status codes defined by the HTTP protocol ${ }^{17}$ Therefore, implementers may provide relevant messages when dealing with clients such as successful requests (2xx), bad requests (4xx), etc. LDA and GraphQL do not

16 https://semver.org/

17 https://www.ietf.org/assignments/http-status-codes/http-status-codes.xml 
provide details about response messages; however, as technologies that implement such specifications are served over HTTP they may reuse HTTP status codes.

\subsubsection{Criteria for comparing technologies and tools for generating APIs}

Table 2.3 describes the criteria proposed in the framework to compare API generation technologies and tools. Since some of these criteria are the same as those defined for comparing specifications in subsection 2.3.3.1, we present below only the new criteria that we included to compare technologies and tools.

Table 2.3: Comparison criteria for technologies and tools

\begin{tabular}{|c|c|}
\hline Criteria & Definition \\
\hline Year & Year when the technology or tool was made available \\
\hline $\begin{array}{l}\text { Interface Description } \\
\text { Language }\end{array}$ & $\begin{array}{l}\text { Specification to document functional and non- } \\
\text { functional aspects of the API }\end{array}$ \\
\hline Input & Files needed for the API generation \\
\hline Output & Result of the technology or tool execution \\
\hline Operations & Allowed methods for managing resources \\
\hline Configuration format & $\begin{array}{l}\text { The file format to be provided with the details of the } \\
\text { technology or tool }\end{array}$ \\
\hline Configurable queries & Support for customized queries \\
\hline Authentication & $\begin{array}{l}\text { Allowed methods to verify the identity of a user or } \\
\text { process }\end{array}$ \\
\hline Resources & $\begin{array}{l}\text { Type of resources allowed such as single, collection, } \\
\text { or nested resources }\end{array}$ \\
\hline Versioning & Support for version management \\
\hline $\begin{array}{l}\text { Control over the } \\
\text { JSON structure }\end{array}$ & Support for JSON management \\
\hline Source & The source code / repository of the technology or tool \\
\hline Last release & $\begin{array}{l}\text { Year when the last version of the technology or tool } \\
\text { was released }\end{array}$ \\
\hline Language & $\begin{array}{l}\text { Programming language used for create the technology } \\
\text { or tool }\end{array}$ \\
\hline
\end{tabular}

The first new criterion considered is the Interface Description Language which outlines which convention is followed by a technology or tool to define APIs. We also assess what is the input required by the technology or tool for generating APIs, e.g., an ontology, queries, etc; and the expected result (output) after executing a given technology or tool (e.g., data formats, API specification file, a server, etc.). In addition, we analyze whether technologies or tools provide control over the JSON structure as it helps us to detect which ones allow users to manage such files. As for the source is 
only an informative column that indicates where the technology or tool code, demo, or repository is available for a technology or tool. Moreover, we are interested in the last release date when a technology or tool was updated in order to know whether it is still maintained. Finally, we also are interested in the language selected for the development of the technology or tool as it may help us to understand if there is a preferred option to implement them.

\subsubsection{Results of comparison of API generation technologies and tools}

Table 2.4 presents the comparison between the technologies and tools according to the criteria described in subsection 2.3.3.3. The symbol "-" indicates that the criterion is not described or detailed in the technology or tool source.

One of the first tool to appear was OpenLink Virtuoso, in the year 2008, which became a popular technology to store and manage RDF data. Since then, several alternatives appeared over the years to ease data consumption by providing interfaces based on the REST paradigm and taking advantage of the HTTP protocol. The most recent tool reported in our survey is Walder, released in 2020, which allows generating APIs for consuming RDF data from several sources, in an effort to integrate decentralized endpoints.

Most of the assessed technologies and tools use as Interface Description Languages (IDL) the specifications presented in subsection 2.3.1. However, some of them support other API description blueprints. For example, JSON-QB API requires users to define the API following an ad-hoc specification (JSON-qb API specification). R4R allows users to manually describe the API but does not restrict the use of a specific IDL (e.g. users can provide an OpenAPI-compliant file). RAMOSE requires users to define a hash-format configuration file that contains the details of the API. Pubby and LODI do not require any IDL as they only provide HTML views of resources.

In general, all included technologies and tools require as input the URL of the SPARQL endpoint and the SPARQL queries needed to implement the allowed API methods, but some technologies and tools differ slightly in their needs. The R4R framework, in addition to the aforementioned inputs, requires users to define JSON templates that allow the responses of SPARQL queries or requested resources to be translated into JSON. The AtomGraph Processor requires an application ontology that must follow the structure described in the Linked Data Templates protocol. Such ontology must define the API details, the SPARQL queries to request matching, and the application behavior. The JSON-QB-API technology only requires the URL of the SPARQL endpoint, as SPARQL queries are generic (designed to handle data described with the Data Cube 
vocabulary) and provided automatically. Apache Marmotta and Trellis have a similar input configuration as both only require the RDF source (e.g. an RDF data dump).

Other tools start from OWL ontologies. For example, OBA and OWL2OAS require an OWL ontology as input, which they convert to an OpenAPI specification. OBA also accepts the URL of a target SPARQL endpoint, as it generates a server to handle the client requests. OBA allows users to specify which classes should be excluded in the final API by using a YAML configuration file, while OWL2OAS requires the ontology to be annotated with an ad-hoc Boolean property to define which classes or properties should be taken into account or not in the API. The Ontology2GraphQL application needs an ontology annotated with the GraphQL meta model; this ontology must be stored in a Virtuoso instance which must also contain the RDF data. Finally, Walder supports any RDF source (RDF dump, Linked Data documents, SPARQL endpoint) as input, as well as the GraphQL-LD queries and the specific JSON-LD contexts required for the execution of API operations.

When executing the analyzed technologies and tools, different types of outputs are generated. Virtuoso, Pubby, Puelia, ELDA, Apache Marmotta, AtomGraph Processor, LODI, Trellis, and Walder provide data results in RDF and non-RDF serializations. Tools such as BASIL, GRLC, OWL2OAS, and OBA generate OpenAPI-compliant APIs, and provide the requested data in JSON format. BASIL and GRLC also provide data in other formats such as CSV, XML, and RDF. Likewise, JSON is the output format for JSON-QB-API, R4R, and OSF results. OBA and OWL2OAS generate the API schemas and paths from an OWL ontology, but OBA is the only tool which generates, without human intervention, the basic SPARQL query templates needed to handle the KG data when executing the API methods. Ontology2GraphQL translates the annotated ontology into a GraphQL schema and provides a GraphQL service implementation; however, the annotation process must be performed manually. Finally, RAMOSE generates an HTML with the API documentation, a dashboard for monitoring the API, and provides data results as CSV or JSON files.

The evaluated technologies and tools also allow different operations to be carried out. Figure 2.7 shows the data management levels that such technologies and tools offer on a scale from full query access to a read only query level. Data management levels depend on the allowed operations, thus technologies and tools that support read-only operations provide less freedom than those that allow query execution at the endpoint level. Trellis and the Solid Community Server also allow HEAD (to ask for information about resources), OPTIONS (to describe the communication options of a resource), and PATCH (to partially update resources) operations. 


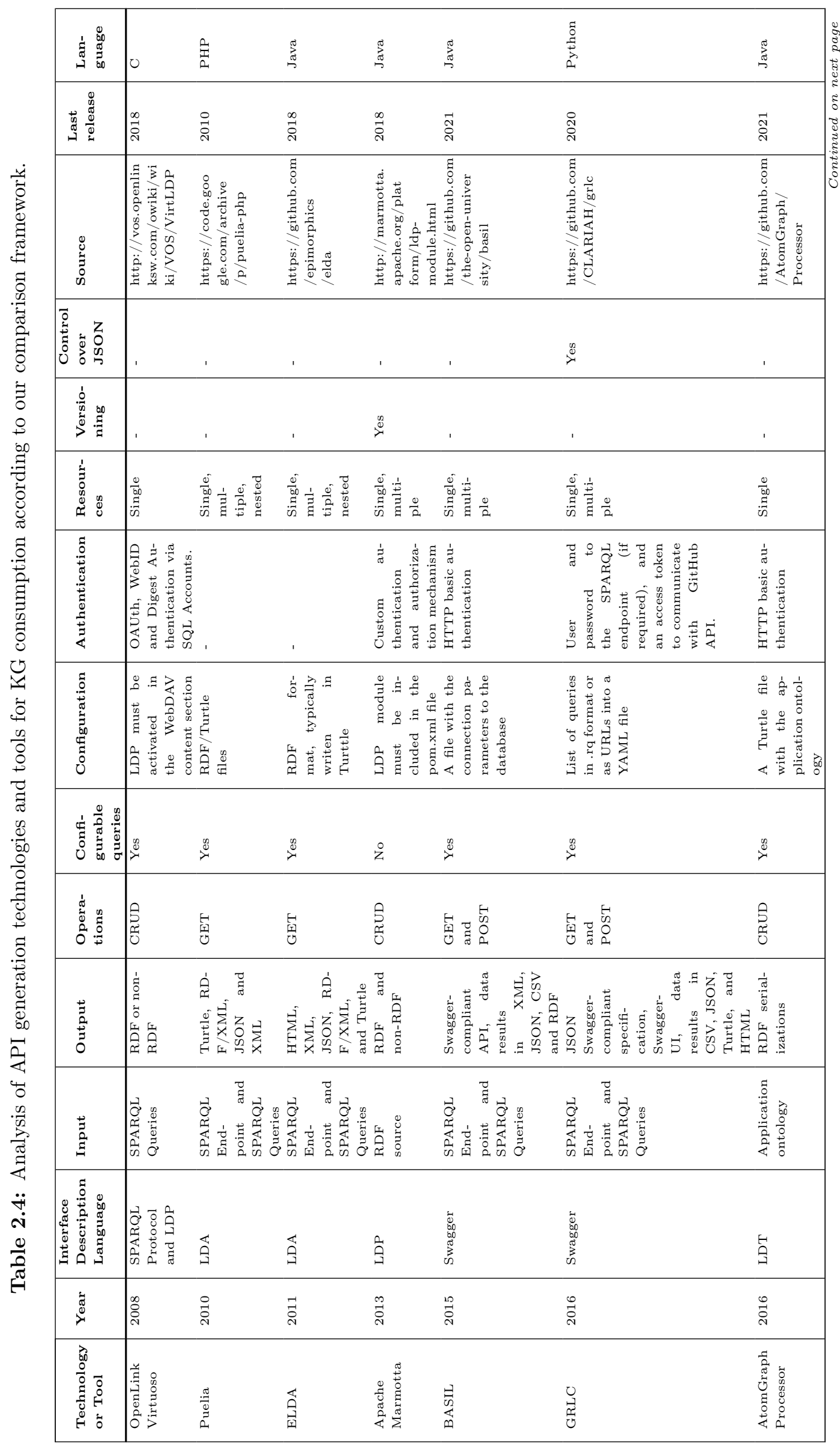




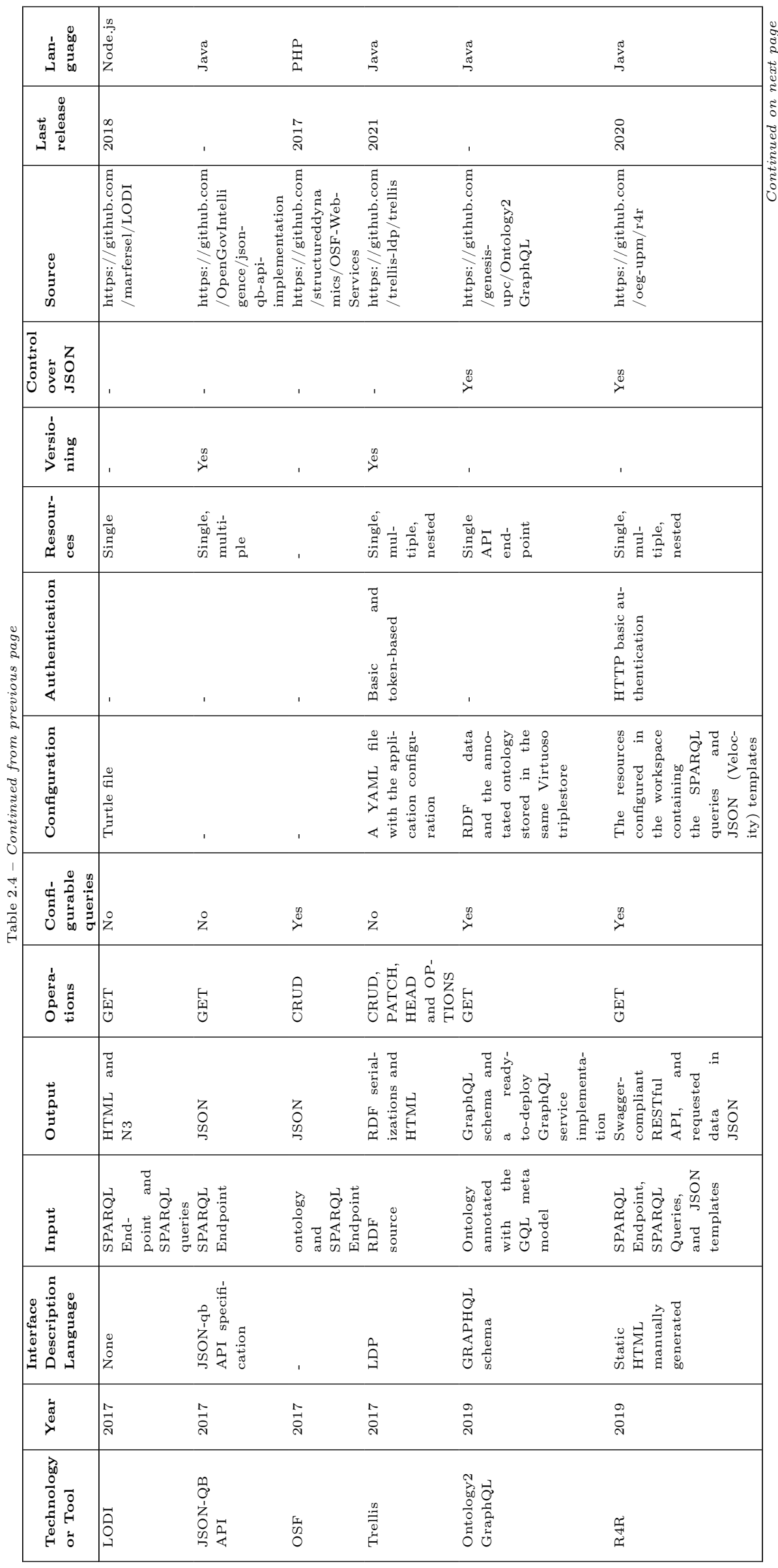




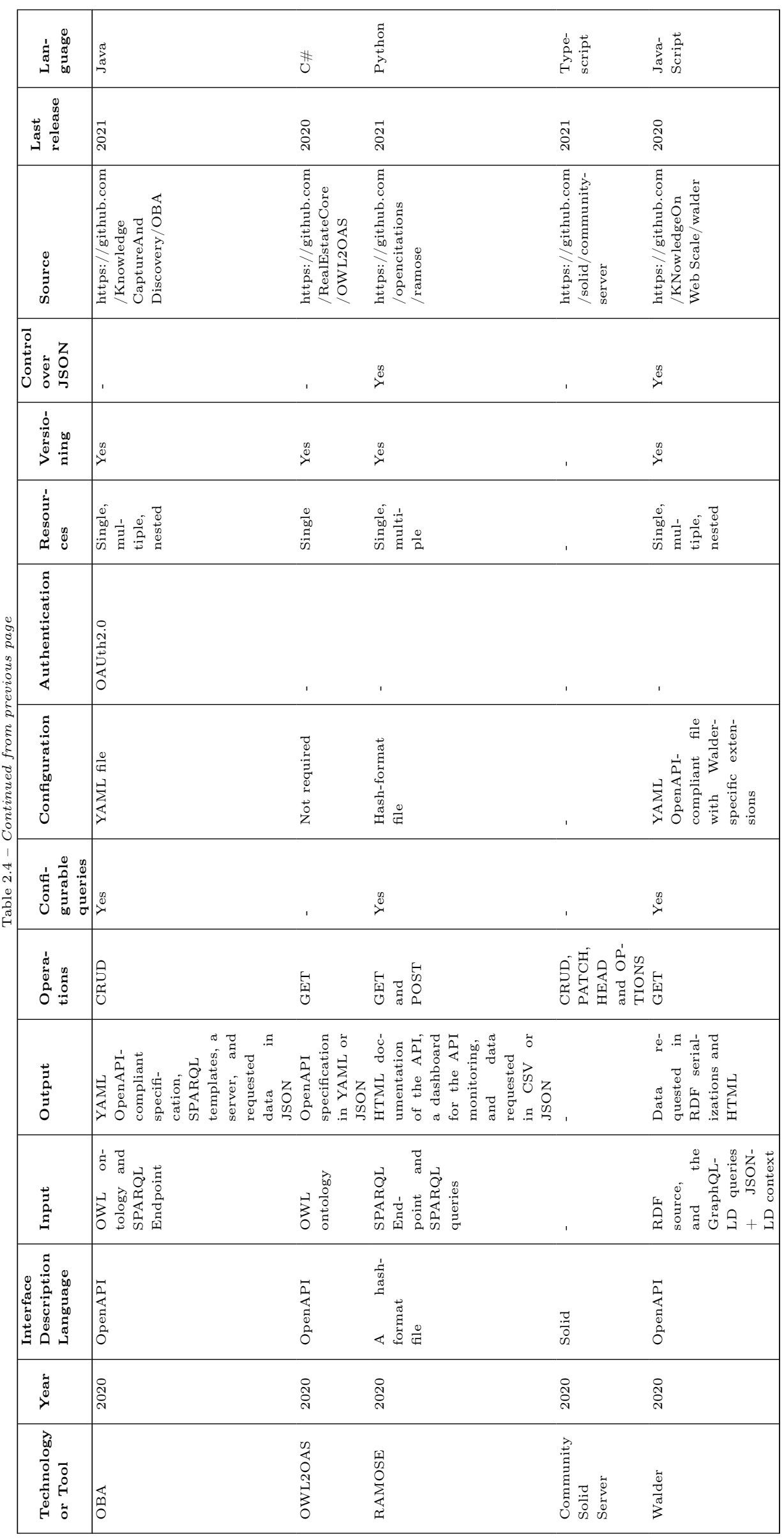




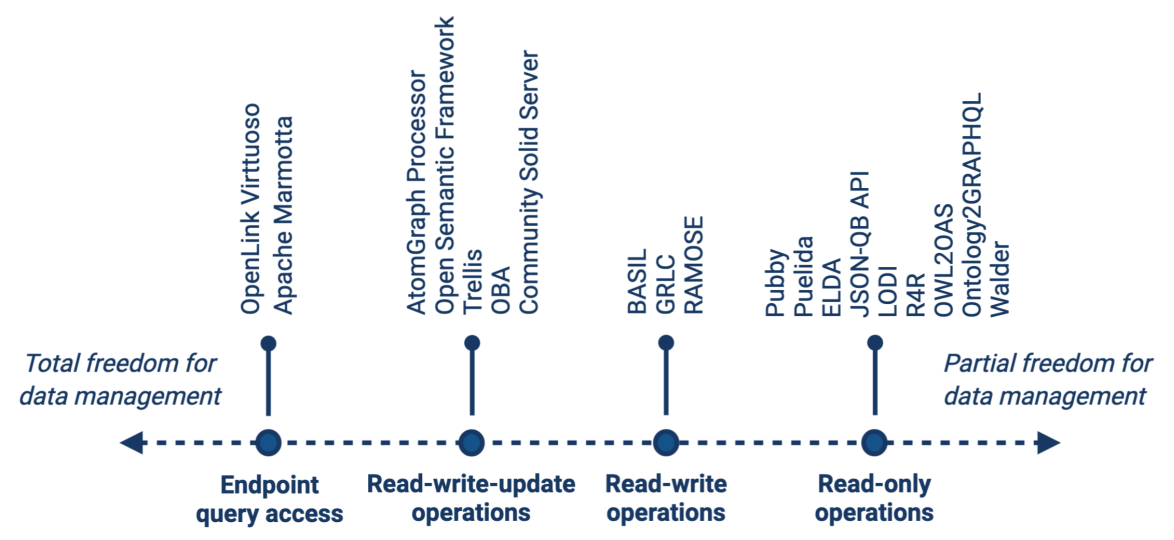

Figure 2.7: Data management levels offered by API generation technologies and tools for KG consumption.

Few technologies and tools provide details on how to manage user authentication. This depends on the allowed operations, since, in general, reading operations do not need to authenticate users. Virtuoso and Marmotta allow complete freedom for data management, and thus provide more details on their authentication methods (OAuth for Virtuoso, ad-hoc authentication and authorization mechanisms for Marmotta ${ }^{18}$ ). Among those technologies and tools allowing writing operations such as update or delete, some of them provide support for authentication mechanisms. For example, basic HTTP authentication is supported by AtomGraph Processor, Trellis, BASIL, and R4R; whereas GRLC requires an access token to communicate with the GitHub API, and the user and password of the SPARQL endpoint, if required. OBA supports OAuth2.0 by default, but authentication can be extended to other methods (which need to be configured by hand).

As for configurable queries, almost all technologies and tools define their own mechanisms to allow users defining custom queries. For example, Basil, GRLC, and OBA use ad-hoc decorators in queries to parametrize them and align them to their exposed APIs; while Ontology2GraphQL and Walder accept GraphQL queries. The analyzed technologies and tools also use different configuration formats. RDF is the most common choice, but Trellis, OBA, and Walder use YAML configuration files. Technologies like Marmotta and Virtuoso, which support LDP, require to activate the LDP mode by providing specific configuration settings applied to a java file (Project Object Model file) and to its configuration utility (Conductor) respectively. BASIL requires a configuration file (.ini) with connection parameters to the database that must be configured

18 https://marmotta.apache.org/platform/security-module.html 
with some required database queries together with a MySQL server. R4R requires to configure the SPARQL queries (.sparql) and JSON templates (.json.vm) both stored into the specific directory that will be taken as the source for the resource path generation. GRLC requires to specify a collection of SPARQL queries (.rq files) into a GitHub repository, but it also allows users to provide such queries as a YAML file containing a list of URLs of SPARQL queries online available. RAMOSE requires a hash-format (.hf) file described according to a simplified version of Markdown syntax.

All the assessed technologies and tools manage single resources. Marmotta, BASIL, GRLC, JSON-QB-API, and RAMOSE also provide collection of resources. Besides single and collection, Puelia, ELDA, Trellis, OBA, R4R, and Walder provide nested resources. Therefore, these last tools allow defining more specific paths for data consumption.

As for versioning, tools like JSON-QB-API, OBA, R4R, OWL2OAS, and RAMOSE allow users to specify the API version in the API documentation, but they do not implement control over different versions. In contrast, Apache Marmotta and Trellis manage data versioning through the Memento protoco 19 a variant on content negotiation which enables accessing a resource version that existed around a specific datetime. Ontology2GraphQL and Walder assume that the server must manage data versioning, and hence do not support versioning.

Only five tools provide control over the JSON structure. GRLC allows developers to pose queries as a JSON object for specifying what data will be retrieved from the endpoint and what shape the results should follow. R4R allows configuring JSON templates that map the SPARQL query results to compose the desired JSON output. RAMOSE also allows users transforming each key-value pair of the final JSON result according to the rule specified in the call URL. Such transformations rules can be used to convert the output into an array or into a JSON object. Lastly, since Ontology2GraphQL and Walder use GraphQL, both allow managing JSON according to the developer needs. This gives more flexibility to developers issuing queries to KGs, but at the same time forces them to be familiar with the ontology used to represent the information in detail.

Most of the analyzed technologies and tools show changes over their last release compared to when they first were made available. Early technologies and tools like Virtuoso, ELDA, and Marmotta have evolved over time in contrast to Puelia, which shows no change. As for Ontology2GraphQL and JSON-QB API, they do not have any release in their source repositories. Since the latest changes observed in the repositories of both tools date from the same year in which they were made available, they may not

${ }^{19}$ https://tools.ietf.org/html/rfc7089 
be currently maintained. Most recent tools have recent releases, which may mean that they are evolving as people begin to use them and new requirements and enhancements are implemented. Finally, regarding the programming languages for the development of technologies and tools, Java is the preferred option (used by 10 implementations), followed by PHP, and Python (each selected by 2 implementations), and lastly C, C\#, JavaScript, Node.js, and TypeScript (each chosen by 1 implemetation).

\subsection{Conclusions and limitations of the State of the Art}

After the description and analysis of the state of the art methodologies for ontology development and the approaches for API generation from ontologies, we conclude this chapter with the main insights that have motivated this thesis.

Several methodologies have been proposed for building ontologies. In general, we can conclude from the methodologies analyzed that all they propose, at a lower or higher level of detail, similar core steps to develop ontologies. At the beginning of the development process, the methodologies propose to identify the requirements that the ontology should fulfill. To do this, all of the methodologies analyzed suggest posing competency questions. Then, the methodologies propose to generate a model or intermediate representation of the ontology that contains the terms and the relationships between them. Subsequently, the methodologies suggest formalizing the model previously generated and, finally, testing whether the formal representation of the ontology answers the competency questions. In addition, some methodologies suggest a maintenance step to allow including changes in the ontology.

Despite the evolution of ontology development methodologies during the years from less detailed descriptions of the steps involved to more documented stages, activities, tasks, guidelines, and techniques for ontology development, there is a lack of details on how to use an ontology after it has been generated.

Regarding the approaches for API generation from ontologies, we can conclude that most of the tools and technologies do not consider ontologies or the ontology artefacts for building these interfaces. Most of the approaches analyzed generates the API from SPARQL queries. However, only OBA and OWL2OAS tools allow generating an OpenAPI specification from the OWL ontology. Moreover, OBA generates automatically SPARQL query templates needed to execute CRUD operations, and it also provides the server side functionality for the API. In addition, we can also conclude that in all the tools and technologies analyzed the effort is focused on the technological support to automatically generate basic APIs rather on a methodology for designing them. 
Therefore, we can state that there is not a methodological approach to generate APIs taking as input the ontology artefacts generated during the ontology development process.

Finally, as a consequence of a missing methodology, there is not a technology which implements the methodological approach to generate APIs that allow ontology-based data consumption. 



\section{Chapter 3}

\section{Objectives and Contributions}

This chapter presents the objectives of this thesis and the open research problem it aims to address (Section 3.1) together with its main contributions (Section 3.2), the thesis assumptions, hypotheses and restrictions (Sections 3.3, 3.4, 3.5, as well as the plan for the evaluation of the stated hypotheses (Section 3.6. The chapter concludes with the research methodology and research process followed during the development of this thesis (Section 3.7.).

\section{$3.1 \quad$ Objectives}

Given a domain where an ontology has been developed following good ontology engineering practices defined by ontology development methodologies, the research question we want to answer in this thesis is: Is it possible to generate APIs based on the artefacts created by ontology engineers during the ontology development process? To address this open research problem, we identify the following objectives:

O1. To help ontology engineers generate APIs to allow ontology usage and Knowledge Graph data consumption.

O2. To automate the API generation process from ontology artefacts generated during the ontology development process.

In order to achieve the first objective, the following research problem must be solved:

- Current approaches for generating APIs to consume KGs have mainly focused so far on the development of technological solutions to generate such interfaces. There is a lack of a formally defined method for API generation that considers 
the experience that the ontology engineer has gained on the target domain and the ontology artefacts that have been generated during the ontology development process. This situation duplicates the effort already done by ontology engineers. Therefore, when someone wants to define a customized API to consume KG data organized according to that ontology they have to begin by understanding the modeling decisions, knowledge domain, ontology implementation code, etc. Our goal is to propose a method that guides ontology engineers in the API generation process using those ontology artefacts as the main inputs for the API design, so that application developers do not need to do extra work.

In order to achieve the second objective the following technological problem must be solved:

- Most tools and technologies to generate APIs for KG data consumption do not consider ontologies for designing APIs, since such tools and technologies are mainly focused on the API generation from SPARQL queries. Recent tools take into account the ontology for the API generation; however, in this process these tools do not consider other ontology artefacts that are usually produced by ontology engineers when they follow good ontology engineering practices, e.g. the competency questions. Therefore, our goal is to provide technological support to help ontology engineers in the API generation process by reusing these intermediate and final ontology artefacts.

\subsection{Contributions to the State of the Art}

The work done in this thesis aims to provide solutions to the goals and problems described in Section 3.1. The contributions for solving the aforementioned objectives are:

C1. A method for API generation, which proposes a set of activities and tasks to define, specify, implement, validate, and deploy APIs based on ontology artefacts. Our method is inspired by Software Engineering practices for designing and building APIs and uses as an initial input the ontology requirements defined during the early phases of the ontology development process. In addition, our method proposes the reuse of other ontology artefacts to ease the API generation process to provide KGs data access such as the SPARQL queries, the ontology code, or the glossary of terms.

With respect to the current state of the art, we will provide a new method that aims at helping ontology engineers in generating APIs. 
C2. A tool for supporting API generation (OATAPI), which allows generating a set of API paths based on two ontology artefacts: competency questions and ontology code. In addition, this tool allows generating the SPARQL queries required to get data to solve the competency questions.

With respect to the current state of the art, this contribution provides a new tool for API generation based on commonly available ontology artefacts.

\subsection{Assumptions}

This thesis was developed under a set of assumptions that help to explain the decisions taken for the achievement of the thesis goals; such assumptions are listed below:

A1. Following good ontology engineering practices, a set of competency questions is defined before implementing any ontology, and these questions are available.

A2. APIs are data consumption interfaces that are well-known by application developers.

A3. The provision of APIs for Knowledge Graph data consumption is a common practice in the Semantic Web field.

A4. Competency questions can be translated into APIs, which provide data required for their expected answers.

A5. Knowledge Graphs for which the APIs are generated contain data organized according to ontologies from which the API has been derived.

A6. Competency questions are written in natural language following state of the art practices.

A7. Ontologies have labels that make their elements human readable.

\subsection{Hypotheses}

Once the assumptions have been identified and presented, the set of hypotheses of this thesis are described:

H1. It is possible to propose a clearly-defined and reproducible method that allows creating APIs from a set of ontology artefacts in such a manner that these APIs allow getting data from KGs. 
H2. It is possible to automatically generate APIs (based on ontology artefacts) that are indistinguishable from the APIs that would be defined by application developers in real use cases.

H3. It is possible to automatically generate APIs (based on ontology artefacts) that are similar to the APIs defined manually according to our proposed method.

\subsection{Restrictions}

Finally, the following restrictions define the limits of the contributions of this thesis and allow proposing future research problems. These restrictions delimit the research problem and allow the incremental improvement of research:

R1. The method for API generation considers only ontologies implemented in OWL.

R2. The competency questions must be written in the English language.

R3. The ontology elements contain labels defined in English.

R4. The main terms used in the competency questions are the same as the labels of the classes, object properties, and datatype properties defined in the ontology.

R5. In ontologies that do not contain labels, the URI of ontology elements must be defined with short and compact natural language names.

R6. The ontology has been evaluated and does not contain errors.

R7. Data and object properties must have a domain and range explicitly defined.

Figure 3.1 summarizes the objectives identified in Section 3.1 and the contributions of this thesis presented in Section 3.2. In addition, this figure presents, for each contribution, relations to the associated assumptions, hypothesis and restrictions (presented in Sections 3.3, 3.4, 3.5.

\subsection{Evaluation Plan}

The hypotheses defined in section 3.4 will be evaluated as follows (see Chapter 5 for all the details of the evaluation performed): 

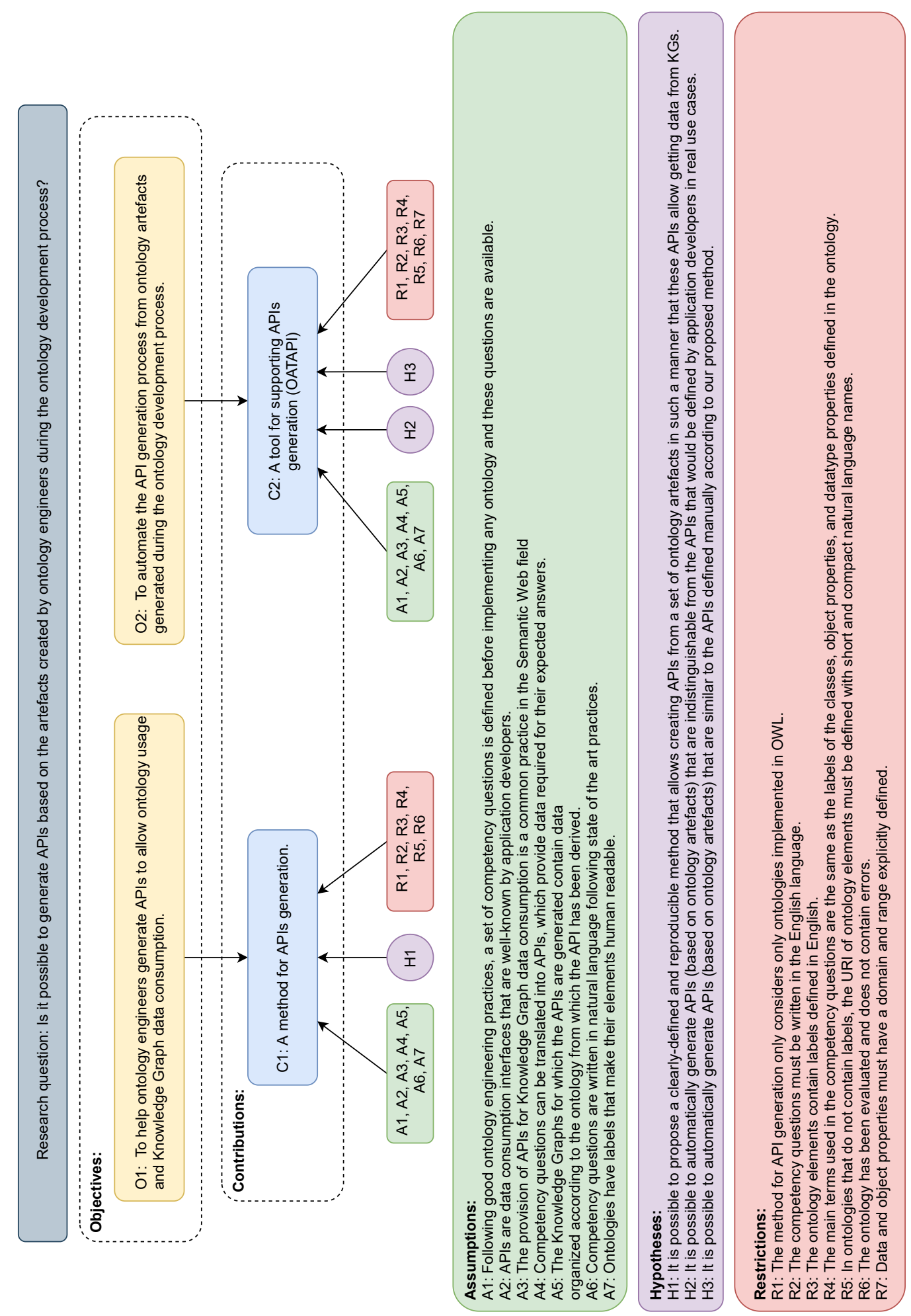

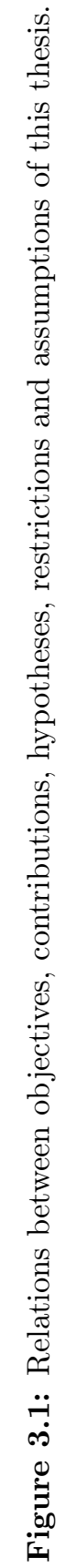


E2 (for H2). The goal of this evaluation is to analyze whether the APIs generated by OATAPI are indistinguishable from those that have been manually defined by application developers. To this end, an empirical evaluation will be carried out to compare the APIs we gathered in the testbed with those resulting from the tool proposed in this thesis. The APIs of the testbed were gathered from the open data portal of the Zaragoza city council and Ciudades Abiertas (Open Cities) project during the Field Research Work phase as presented in Section 3.7

E3 (for H3) The goal of this evaluation is to determine whether OATAPI is able to generate API paths that are similar to manually defined ones. To this end, an empirical evaluation will be carried out to compare the APIs from the corpus developed manually with those resulting from the tool proposed in this thesis. This corpus was defined during the Development phase as presented in Section 3.7, and the APIs from this corpus were generated according to the method proposed in this thesis.

\subsection{Research methodology}

This section presents an overview of the research methodology followed during the development of this thesis. To develop the contributions associated with the research problems defined in Section 3.1, we defined several phases as part of the methodology summarized in Figure 3.2. This figure depicts the phases, the activities carried out, and the contributions generated in each phase. These contributions are cited with integers between brackets that correspond to the enumerated bibliographical publications at the bottom of this figure. In the following, the four phases are described:

1. Field Research Work. During this phase, several ontologies were developed (in the domains of business, budget, traffic, demographics, among others) to analyze and understand the ontology development process and the artefacts that result from such ontology development process. In addition, supervision work was carried out to help application developers to define APIs to provide access to data described with ontologies. As a result of this observation and experimental work, we identified the research problem regarding the lack of a methodological approach for API generation that considers the ontology artefacts and the domain knowledge acquired by the ontology engineer (related to C1). In addition, we collected the APIs defined in this phase as part of a testbed for our evaluation purposes (related to $C 1$ ). 
2. Survey. During this phase, several specifications, tools, and technologies for API generation to consume KG data were studied and compared to identify the existing approaches. As a result, we note that there is no method to define and build APIs based on ontology artefacts (related to $C 1$ ). In addition, we did not find a tool to support the execution of the activities required for the generation of these APIs (related to C2).

3. Development. During this phase, the method for API generation was defined. As a result, we proposed a set of activities to define, specify, implement, validate, and deploy APIs reusing ontology artefacts (related to $C 1$ ). In addition, we did not find a tool to support the execution of the activities required for the generation of these APIs (related to C2). We also generated a corpus of API paths designed according to the proposed method (related to C2).

4. Implementation. During this last phase, existing programming libraries were used to implement OATAPIs, a tool aiming at supporting the API generation process. Moreover, the initial version of the method developed in the previous phase was refined (related to C1). As a result, we released an open-source technological solution (related to C2). 


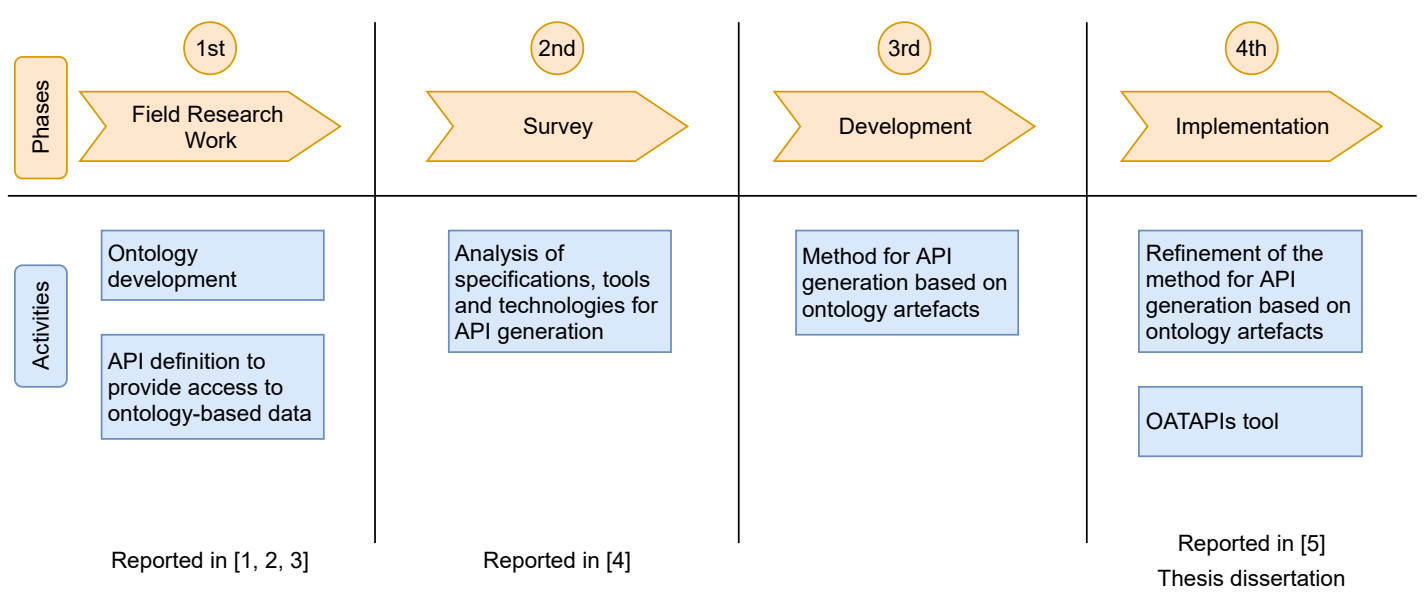

[1] Espinoza-Arias, P., Poveda-Villalón, M., and Corcho, O.. Using LOT methodology to develop a noise pollution ontology: a Spanish use case. J Ambient Intell Human Comput 11, 4557-4568 (2020).

[2] Espinoza-Arias, P., Fernández-Ruiz, M.J., Morlán-Plo, V., Notivol-Bezares, R. and Corcho, O. The Zaragoza's Knowledge Graph: Open Data to Harness the City Knowledge. Information (2020), 11, 129.

[3] Poveda-Villalón M., Espinoza-Arias P., Garijo D., Corcho O. (2020) Coming to Terms with FAIR Ontologies. In: Keet C.M., Dumontier M. (eds) Knowledge Engineering and Knowledge Management. EKAW 2020. Lecture Notes in Computer Science, vol 12387. Springer, Cham.

[4] Espinoza-Arias, P., Garijo, D. and Corcho, O.. Crossing the Chasm Between Ontology Engineering and Application Development: A Survey. Journal of Web Semantics. 70 (2021).

[5] Espinoza-Arias P., Garijo D. and Corcho O. (2020) Mapping the Web Ontology Language to the OpenAPI Specification. In: Grossmann G., Ram S. (eds) Advances in Conceptual Modeling. ER 2020. Lecture Notes in Computer Science, vol 12584. Springer, Cham.

Figure 3.2: Phases of the thesis development, including the activities carried out during each phase and the main publications derived from each phase. 


\section{Chapter 4}

\section{A method for API generation based on ontology artefacts}

This chapter presents our proposed method for API generation based on ontology artefacts, together with its technological support. This method aims at involving ontology engineers and application developers in a new phase in the ontology development process that may be included in the last stages of the traditional ontology development methodologies: the ontology exploitation phase. This method is inspired by common practices adopted in the Software Engineering field and provides details on how to generate interfaces to define a set of resources that provide support to the initial requirements of the ontology.

\subsection{Activities within the API generation method based on ontology artefacts}

In this section we describe the activities to be carried out to generate APIs from ontology artefacts. In the literature, existing ontology development methodologies (e.g. LOT, NeOn, SAMOD, among others presented in section 2.2) usually involve phases such as requirements specification, ontology implementation, ontology publication, and maintenance. However, none of these methodologies pay much attention to how the ontology will be consumed after publication. Therefore, we propose a new ontology exploitation phase to be considered in the process described by these methodologies. This new phase encompasses any task where the ontology must be used; therefore, it may include tasks such as RDB2RDF mapping definition, RDF data generation, and data consumption mechanisms provision, among others. 
In this thesis, we will focus especially on the provision of data consumption mechanisms through APIs, as none of the well-established methodologies provide details on how to help ontology engineers to generate APIs for KG data consumption by application developers. Therefore, the ontology exploitation phase will describe the details for the API generation process. Figure 4.1 illustrates this phase as an extension of the LOT methodology (Poveda-Villalón et al., 2019). However, the ontology exploitation phase that we propose may be easily adopted by any methodology, as the proposed phase is general enough, as it makes use of resources that are commonly generated in all these methodologies. It is worth mentioning that in this thesis, for readability reasons, the main LOT's activities are called phases. Thus, we can describe the methodology as a set of phases involving activities, these activities involve several tasks, and so on.

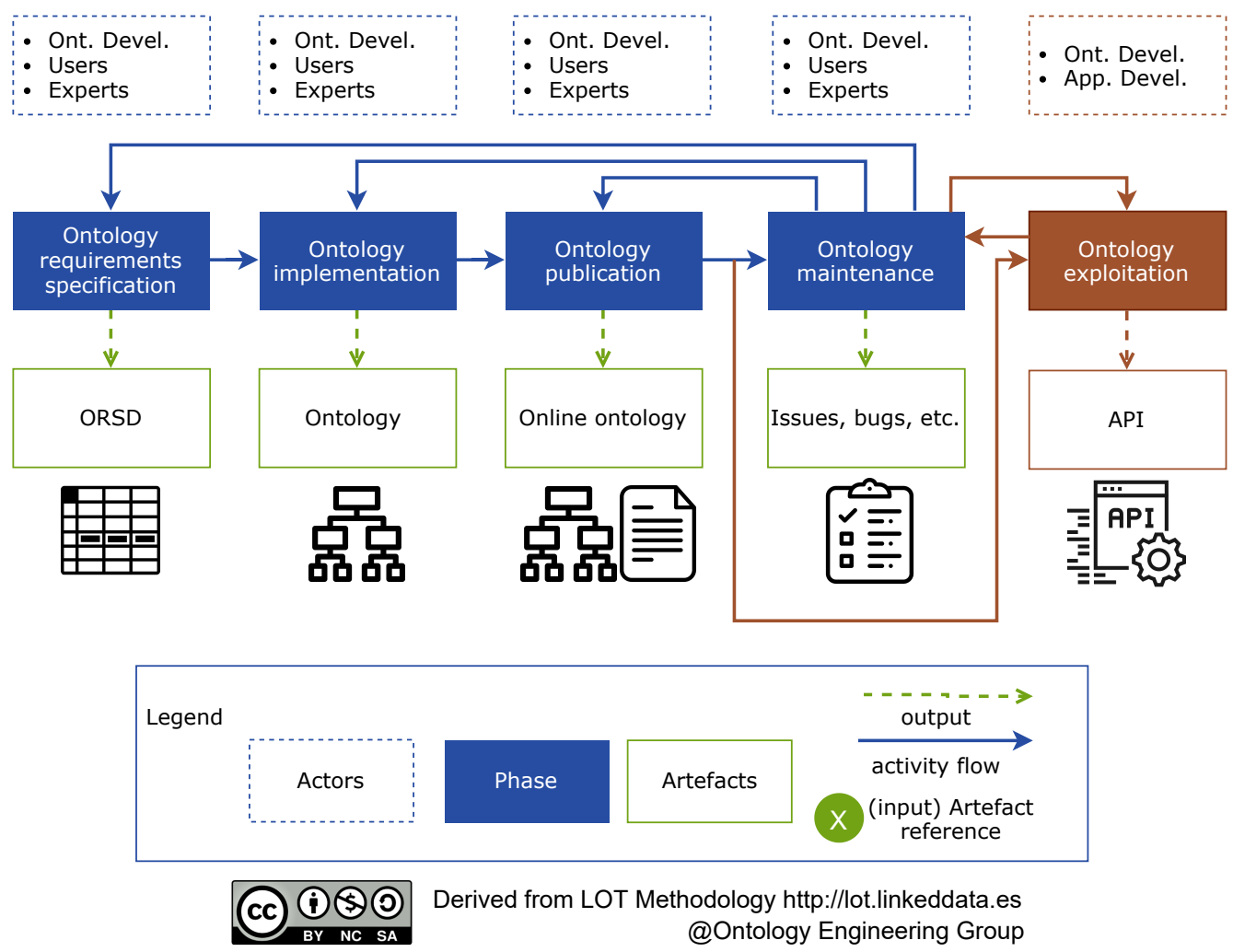

Figure 4.1: Ontology exploitation phase as an extension of the LOT Methodology. New elements are illustrated in brown.

As depicted in Figure 4.1, the actors involved in the ontology exploitation phase are ontology developers (aka ontology engineers) and application developers. In addition, the inputs of the phase are the ontology artefacts produced during the previous phases 
of the ontology development process, and the output is an API. Finally, if a bug is detected or an update is required the maintenance phase should be triggered and a new iteration of the ontology development process should begin.

In order to define the APIs (including the resources to be covered, the operations to be provided, the responses to be delivered, among others), we design a method that proposes a set of activities to generate APIs from ontology artefacts. Our method is inspired in the workflow for designing and building Web APIs presented in (Jin et al., 2018). The following sections describe each of these activities, which are summarized in Figure 4.2

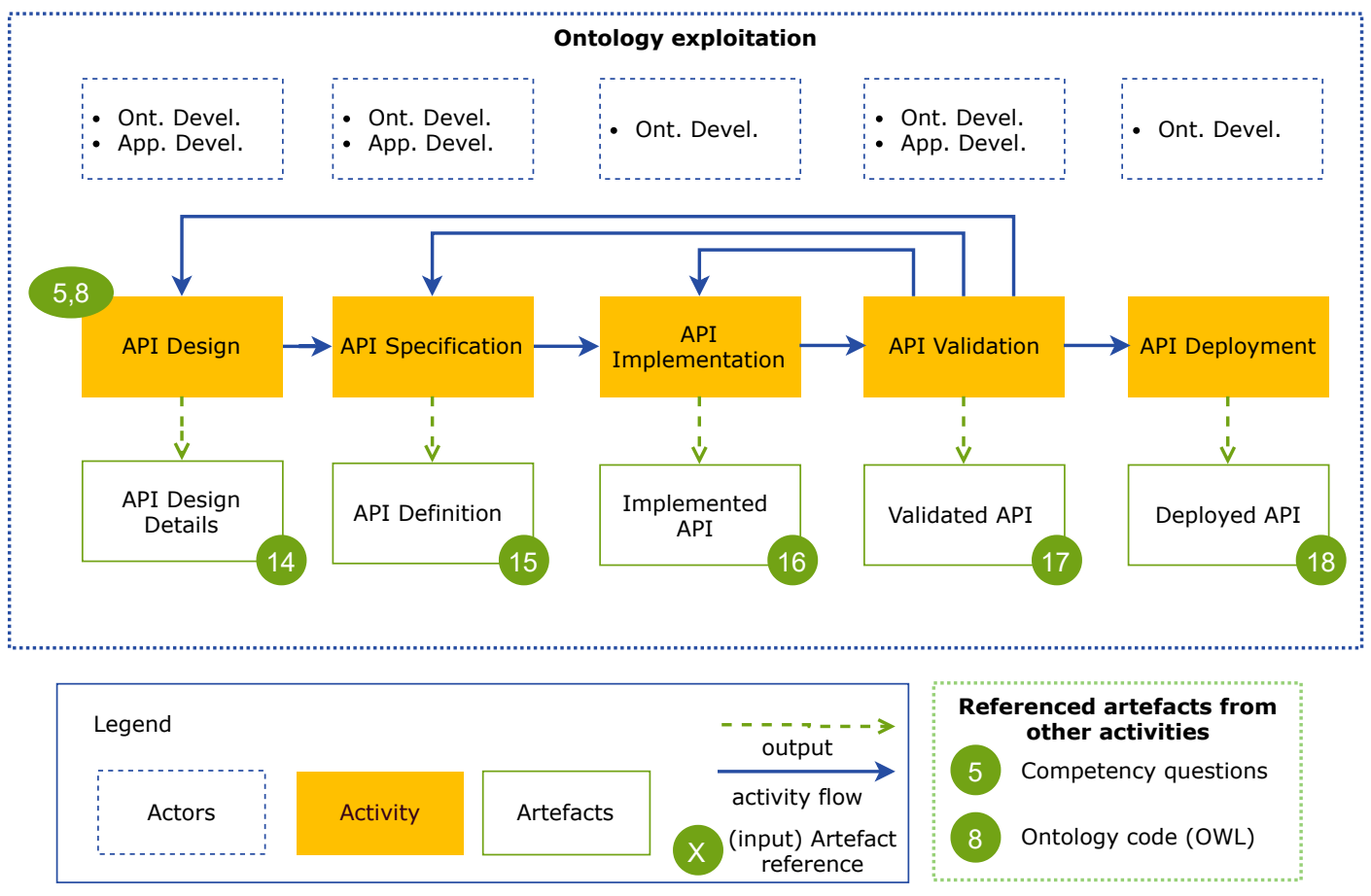

Figure 4.2: Activities of the method to generate APIs based on ontology artefacts (This figure follows the convention defined by the LOT methodology.)

\subsubsection{API design activity}

This activity focuses on deciding how the API will behave and defining the resources along with the operations that the API will provide. To this end, we propose a set of tasks to guide ontology engineers and application developers in making these decisions and defining resources and operations taking ontology artefacts as the main input. 
Figure 4.3 shows the inputs and outputs of the tasks involved in this activity. Each task is described in the following subsections. For illustrating purposes throughout this chapter, we will be using as example the local business census ontology $\sqrt{20}$ along with its competency questions ${ }^{21}$ generated during the Field Research Work phase presented in section 3.7. We will also use the Videogame ontology ${ }^{22}$ and its competency questions ${ }^{23}$ which we use to generate the corpus mentioned in the Development phase presented in section 3.7 .
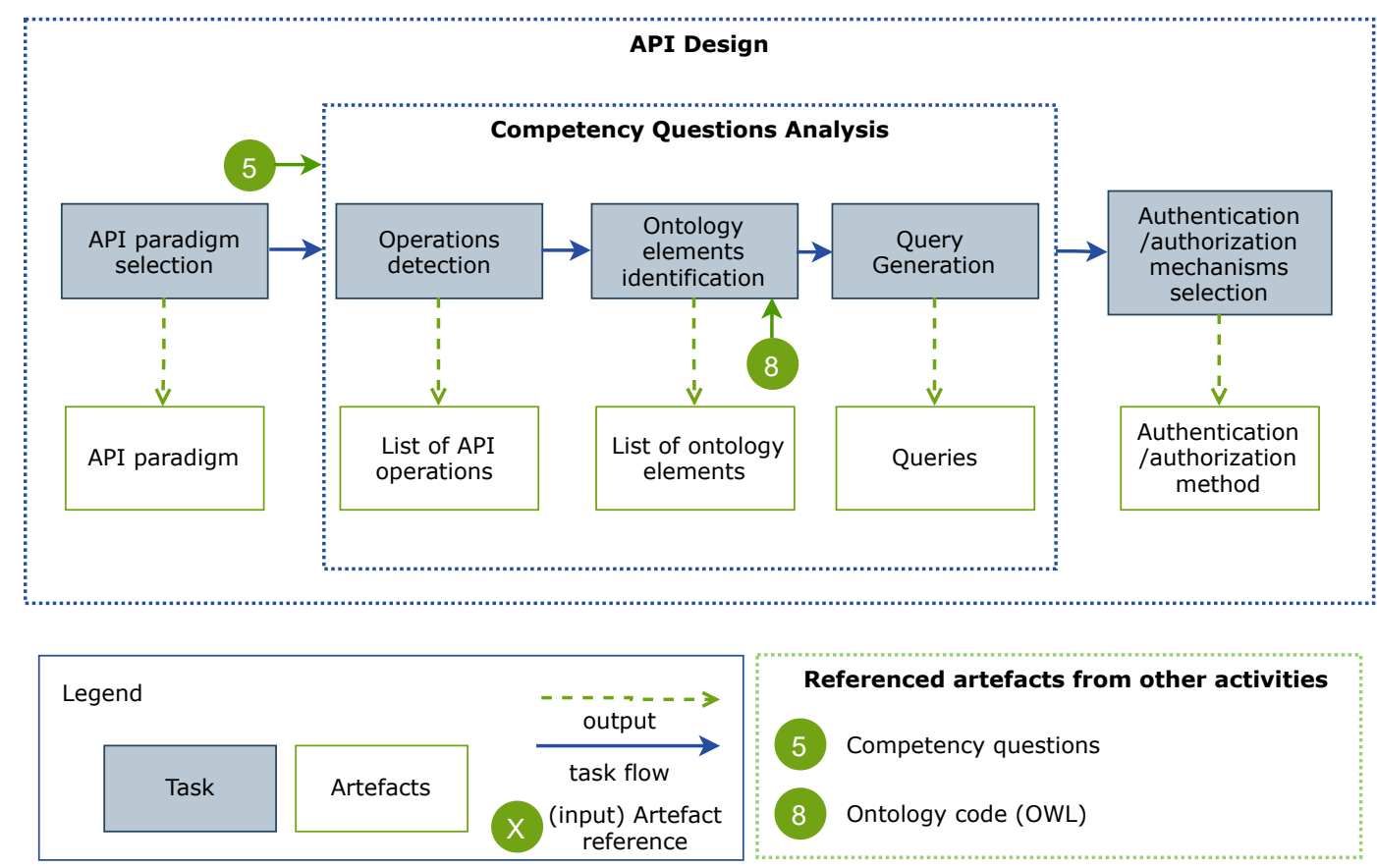

Figure 4.3: Tasks involved in the API design activity

\subsubsection{API paradigm selection}

The goal of this task is to choose the API paradigm to design the desired interface. The selection on which paradigm to use will depend on the decision of ontology engineers and application developers, after an analysis on the pros and cons of the existing paradigms and how they fit in the requirements that will need to be addressed by the ontology,

\footnotetext{
20 http://vocab.ciudadesabiertas.es/def/comercio/tejido-comercial

${ }^{21}$ https://github.com/CiudadesAbiertas/vocab-comercio-censo-locales/tree/ master/requirements

22 http://vocab.linkeddata.es/vgo

23 https://doi.org/10.5281/zenodo.1967306
} 
maintainability, consumers, a specific application behaviour, etc. The selected API paradigm must be a request-response one such as those oriented to a resource-style, hypermedia-style, query-style, among others. In the Semantic Web community the most common paradigms adopted are REST and GraphQL, which are resource-style and query-style oriented paradigms respectively (Espinoza-Arias et al., 2021). Therefore, in this method we present details on how to carry out the activities and tasks according to both paradigms.

To illustrate the execution of this task, let us assume we select the REST paradigm to generate an API for the CQs and ontologies of our example (from the local business census and video game domains previously introduced). Therefore, this resource-oriented paradigm will require defining the resources to be exposed, the API paths, the responses structure, etc. as we will explain in the following subsections

\subsubsection{Competency questions analysis}

This task aims to analyze the competency questions, produced during the ontology specification activity, to find the relevant terms required for the API design. Three subtasks should be executed, as follows.

1. Operations detection. This subtask aims to identify which operations will be implemented in the API according to the intent of the competency questions. To detect these operations, the ontology engineer must analyze specific terms that request something in the competency questions. Table 4.1 shows some examples of common terms and their correspondence with the operations from the REST and GraphQL paradigms. In general, competency questions consider read-only operations rather than requests for data changes. However, if a competency question intends to perform data changes the operation detection should also consider terms related to write operations.

Table 4.1: Common competency questions terms denoting operations in APIs.

\begin{tabular}{|l|l|l|}
\hline \multicolumn{1}{|c|}{ Paradigm } & REST & GraphQL \\
\hline $\begin{array}{l}\text { get, list, which, what, who, } \\
\text { where, when, obtain, give }\end{array}$ & GET & query \\
\hline add, insert & POST & mutation \\
\hline update, change & PUT & mutation \\
\hline delete, remove & DELETE & mutation \\
\hline
\end{tabular}

It is worth mentioning that in the case of competency questions aiming to solve 
boolean or counting questions, it will be necessary to adopt another strategy. To solve these queries, competency questions can be related to a read operation whose resulting data must be properly processed to provide the required responses. Table 4.2 shows examples of common terms defining these queries and their correspondence with operations according to each API paradigm. Therefore, to solve counting questions, API consumers can count the resulting data on the client-side to get the requested number of elements. In addition, to solve boolean questions, API consumers can analyze the resulting data to determine that the query response is true, if the results number is less than zero, or false, if it is zero.

Table 4.2: Example of operations detection for counting and boolean queries

\begin{tabular}{|l|l|l|l|}
\hline Term & Query type & REST & GraphQL \\
\hline how many & counting & GET & query \\
\hline is, was, were & boolean & GET & query \\
\hline
\end{tabular}

Following our example above, we analyze some CQs to detect the REST operations they require. For each CQ we check if there is a term that matches those presented in Table 4.1. Then, for each equivalence we identify the REST operation required for the CQ. Table 4.3 presents the CQs and the operations identified. Terms that match operations are shown in bold in the CQs. Moreover, the Id column identifies the CQs of the local business census ontology with the ESCOM acronym and those of the videogame ontology with VGO.

Table 4.3: Operations detection example

\begin{tabular}{|l|l|l|}
\hline Id & Competency Question & REST operation \\
\hline ESCOM1 & $\begin{array}{l}\text { Obtain the information of certain local } \\
\text { business. }\end{array}$ & GET \\
\hline ESCOM2 & $\begin{array}{l}\text { Give me the details of the terrace that } \\
\text { belongs to a local business. }\end{array}$ & GET \\
\hline VGO1 & What achievements does a game have? & GET \\
\hline VGO2 & Who is the creator of the game? & GET \\
\hline
\end{tabular}

2. Ontology elements identification. This subtask intends to distinguish which concepts will be covered by the API. To this end, this subtask requires providing the ontology serialization as input to verify whether the labels of the ontology elements match the terms identified in the CQs. This verification is essential to detect how these concepts are related and, depending on the operation, the 
structure of the expected API input/output. An alternative way to identify the ontology elements may be to take their URIs and analyze their last fragment identifier. However, this alternative does not work if URIs follow an opaque strategy (which obfuscates the name of classes and properties of the ontology) because it makes it difficult to interpret ontology elements (Garijo and PovedaVillalón, 2020). Therefore, to apply this alternative the URIs of ontology elements must be defined with short and compact natural language names.

Finally, in those cases where the terms identified do not match ontology elements it would be necessary to check for their synonyms. To do this, the ontology engineer may reuse the terminology provided in the Glossary of Terms, an ontology artefact that is usually generated to extract key terms and definitions from the documents, data, requirements, among other inputs analyzed during the ontology specification activity. Thanks to this glossary, some terms found in the CQ can be compared with those in the glossary to find the corresponding ontology elements. Another option to identify the terms would be to use existing open glossaries (domain dependent or independent). However, it is advisable to use the same ontology elements in the CQs.

Following our example above, we have to identify the ontology elements from the CQs. For each CQ we check if there is a term that matches the label of the element from the corresponding ontology serialization. Table 4.4 presents the labels of the ontology elements identified from the CQs. For each CQ, classes are shown in bold and properties are presented in italics.

Table 4.4: Example of ontology elements identification

\begin{tabular}{|l|l|l|l|}
\hline \multirow{2}{*}{ Id } & \multirow{2}{*}{ Competency Question } & \multicolumn{2}{|c|}{ Ontology element } \\
\cline { 3 - 4 } & & Class & $\begin{array}{l}\text { Object } \\
\text { property }\end{array}$ \\
\hline ESCOM1 & $\begin{array}{l}\text { Obtain the information of certain } \\
\text { local businesses. }\end{array}$ & Local Business & \\
\hline ESCOM2 & $\begin{array}{l}\text { Give me the details of the ter- } \\
\text { race that belongs to a local } \\
\text { business. }\end{array}$ & $\begin{array}{l}\text { Local Busi- } \\
\text { ness, Terrace }\end{array}$ & $\begin{array}{l}\text { belongs to lo- } \\
\text { cal }\end{array}$ \\
\hline VGO1 & $\begin{array}{l}\text { What achievements does a } \\
\text { game have? }\end{array}$ & $\begin{array}{l}\text { Achievement, } \\
\text { Game }\end{array}$ & $\begin{array}{l}\text { has achieve- } \\
\text { ment }\end{array}$ \\
\hline VGO2 & Who is the creator of the game? & Game & has creator \\
\hline
\end{tabular}

In addition, as our example follows the REST paradigm, it is necessary to define the API paths required to access the resources that answer the CQ. To this end, 
we apply the resource naming practices for REST APIs we have adapted to the ontology elements, these practices are summarized in Annex A. Table 4.5 shows the API paths we generate for our CQs. Note that the last part of these paths determines which resource the API will provide. In addition, observe that we include the Agent class that is not explicitly included in VGO2, but this class can be retrieved from the ontology serialization as Agent is the property range of creator whose domain is Game class.

Table 4.5: Example of ontology elements identification and API paths generation

\begin{tabular}{|c|c|c|c|c|}
\hline \multirow{2}{*}{ Id } & \multirow{2}{*}{ Competency Question } & \multicolumn{2}{|c|}{ Ontology element } & \multirow{2}{*}{ API path } \\
\hline & & Class & $\begin{array}{l}\text { Object } \\
\text { property }\end{array}$ & \\
\hline ESCOM1 & $\begin{array}{l}\text { Obtain the information of } \\
\text { certain local business. }\end{array}$ & $\begin{array}{l}\text { Local } \\
\text { Business }\end{array}$ & & $\begin{array}{l}\text { /local-businesses/ } \\
\text { \{local-business-id }\}\end{array}$ \\
\hline ESCOM2 & $\begin{array}{l}\text { Give me the details of the } \\
\text { terrace that belongs to } \\
\text { a local business. }\end{array}$ & $\begin{array}{l}\text { Local } \\
\text { Business, } \\
\text { Terrace }\end{array}$ & $\begin{array}{l}\text { belongs to } \\
\text { local }\end{array}$ & $\begin{array}{l}\text { /local-businesses/ } \\
\{\text { local-business-id }\} / \\
\text { terraces }\end{array}$ \\
\hline VGO1 & $\begin{array}{l}\text { What achievements } \\
\text { does a game have? }\end{array}$ & $\begin{array}{l}\text { Achievem- } \\
\text { ent, Game }\end{array}$ & $\begin{array}{l}\text { has achie- } \\
\text { vement }\end{array}$ & $\begin{array}{l}\text { /games/\{game- } \\
\text { id\}/achievements }\end{array}$ \\
\hline VGO2 & $\begin{array}{l}\text { Who is the creator of the } \\
\text { game? }\end{array}$ & $\begin{array}{l}\text { Game, } \\
\text { Agent }\end{array}$ & $\begin{array}{l}\text { has cre- } \\
\text { ator }\end{array}$ & $\begin{array}{l}\text { /games/\{game- } \\
\text { id\}/ agents }\end{array}$ \\
\hline
\end{tabular}

3. Query generation. This subtask aims to define the query to manage the data and perform the functionality required by the competency question. To this end, the SPARQL queries generated for the ontology validation may be reused, if available. This validation strategy is common in ontology development methodologies as it allows ontology engineers to verify whether the ontology fulfills the requirements against a proof of concept dataset. However, if queries had not been defined or are not available they must be generated. Therefore, for the query generation, two steps should be carried out. First, take the operation, detected in the first subtask of this activity, and determine what SPARQL operation should be included in the query. Second, match the ontology elements, detected in the second subtask of this activity, with the query structure and format the query accordingly. The query structure should be defined according to the competency query intention and its expected answer.

Following our example above, once we have identified the operations and the ontology elements required for each CQ, we generate the SPARQL query needed for retrieve the expected resources. Listing 4.1 shows queries for ESCOM1 and 
VGO2. In both queries, the parameters ?local-business-id and ?game-id will be replaced with the value specified in the request as they correspond to the \{local-business-id\} and \{game-id\} templates defined in the API paths. Moreover, as the operation detected in both cases is a GET, the SPARQL operation corresponds to SELECT. In addition, these queries also include a CONSTRUCT statement, since the result of the request will be composed of all the values of the properties of each instance found. Finally, it is worth noting that the structure of both queries is similar but the intention of each is different. In the first case, the query intends to get the information of a specific local business, while in the second case, the intention is to get the achievements of a specific game. Therefore, the general structure of SELECT queries could be generalized and reused to generate queries, but its final structure will depend on the CQ intention and the ontology elements detected.

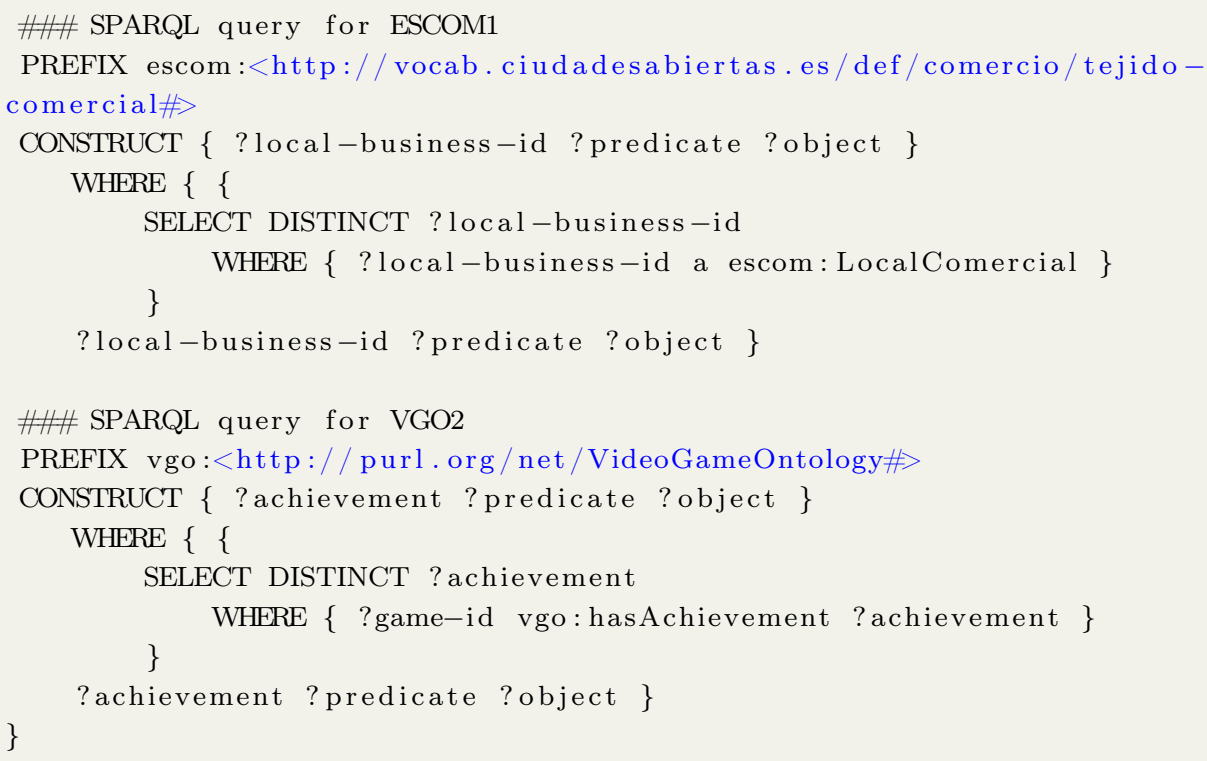

Listing 4.1: SPARQL queries for the ESCOM1 and VGO2 competency questions 


\subsubsection{Authentication and authorization mechanisms selection}

This task aims to define which security mechanisms the API must handle. Depending on operations and data that the API will manage, it may be necessary to provide a security level to control who is allowed and what functions they may execute. For example, it may be necessary to restrict personal data access, or write operations may be allowed only to certain users. The security methods to be provided may be basic (e.g. HTTP requests including username and password) or more reliable (e.g. OAUth 2.0 protoco ${ }^{24}$ which is based on the provision of a token access).

Following our illustrative example, we need to define which authentication and authorization mechanism our API will handle. However, since the CQs in our example only involve read operations, we decide not to use a security mechanism.

\subsubsection{API specification activity}

This activity aims to describe in detail all the previous decisions taken on the API design. To this end, a common practice is building the API specification according to an Interface Description Language (IDL). This language allows documenting functional and non-functional aspects of the API according to a specific syntax provided in a machinereadable format. Using an IDL makes it possible to generate the API documentation very quickly because the specification described with such language can be translated into, for example, an HTML document that includes interactions with the API that can show how requests work. In REST APIs there are plenty of IDLs to describe an API, for example, OpenAPI ${ }^{25}$ JSON API ${ }^{26}$ API Blueprint ${ }^{27}$ among others.

However, other specifications can be adopted by the ontology engineer and application developer as long as they provide facts about the exposed resources, allowed methods, message codes that the API handle, result formats, expected inputs/outputs, relevant metadata, among others. It is worth providing a good specification to help application developers to learn and understand how the API works. Therefore, the easier the generated APIs are to understand, the more developers are drawn to adopt them.

Following our example above, let us assume that we write the API specification according to an ad hoc specification, i.e. we did not follow an IDL. To this end, we designed a specification template (provided in Annex B that allows describing several details of the API that we consider relevant to illustrate what information needs to be

\footnotetext{
24 https://oauth.net/2

25 https://swagger.io/resources/open-api

26 https://jsonapi.org

27 https://apiblueprint.org
} 
supplied. Table 4.6 presents an example of this template filled with the API information for the local business census ontology.

Table 4.6: Example of an API specification for the local business census ontology.

\begin{tabular}{|l|l|}
\hline \multicolumn{2}{|l|}{ General information } \\
\hline API name & Local Business Census API \\
\hline API version & 0.1 \\
\hline Base URL & http://api.example.com \\
\hline API description & $\begin{array}{l}\text { This API provides the operations which allows answering the competency } \\
\text { questions defined for the development of the Local Business Census ontol- } \\
\text { ogy. }\end{array}$ \\
\hline License & https://www.apache.org/licenses/LICENSE-2.0.html \\
\hline Authorization & Accessing this API does not require any authorization mechanism. \\
\hline Contact & support@example.com \\
\hline
\end{tabular}

\begin{tabular}{|c|c|c|c|}
\hline \multicolumn{4}{|c|}{ Details of each API path } \\
\hline API path & Operation & Input & Output \\
\hline $\begin{array}{l}\text { /local- } \\
\text { businesses/\{local- } \\
\text { business-id }\end{array}$ & GET & local-business-id & $\begin{array}{l}\text { a local business instance with spe- } \\
\text { cific id (it will retrieve its attribute } \\
\text { values of business sign, capacity, } \\
\text { cadastral reference, among others.) }\end{array}$ \\
\hline $\begin{array}{l}\text { /local- } \\
\text { businesses/\{local- } \\
\text { business- } \\
\text { id } / \text { terraces }\end{array}$ & GET & local-business-id & $\begin{array}{l}\text { an array of terrace instances (each } \\
\text { instance will retrieve its attribute } \\
\text { values of area, table number, open- } \\
\text { ing hours, number of authorized } \\
\text { chairs, among others.) }\end{array}$ \\
\hline
\end{tabular}

\begin{tabular}{|l|l|l|}
\hline Status codes & Description & Detail \\
\hline Code & OK & The request succeded. \\
\hline 200 & Bad request & $\begin{array}{l}\text { The request cannot be accepted be- } \\
\text { cause the server detect a syntax er- } \\
\text { ror. }\end{array}$ \\
\hline 400 & Not found & $\begin{array}{l}\text { The requested resource was not } \\
\text { found }\end{array}$ \\
\hline 500 & Server error & $\begin{array}{l}\text { Somethig wrong happen on the } \\
\text { server side }\end{array}$ \\
\hline
\end{tabular}

Regarding the sections of the template presented in Table 4.6, the general information section contains several fields that allows describing metadata such as the name, version, description, license, and the base URL of the API. In addition, the authorization field allows describing whether credentials or token are or not required for consuming 
the API. Moreover, the contact field allows providing information on who contact for API support. As for the details of API path section, it allows listing the paths and the available operations for each path together with their inputs and outputs. Finally, the status codes section allows defining which code numbers will be delivered to application developers based on the status of the executed operation. These codes should be detailed together with its description and detail.

As for the information provided in sections of Table 4.6, in the general section we provide the metadata of the Local Business Census API. Then, in the details of each API section we provide two API paths and specify GET as the operation that will allow getting data to solve the competency questions (ESCOM1 and ESCOM2) of the local business census ontology. In the input column we specify that both API paths require the identifier of the local business and in the output column we provide a list of the resource attributes that will be delivered by the API. These attributes correspond to those defined in the ontology class that was previously identified for each CQ in subsection 4.1.1.2. However, these attributes will be delivered to application developers in some serialization format, e.g. JSON. Finally, in the status codes section we provide a list of codes that the API will manage so that developers can understand whether a request fails due to something they did wrong or due an issue on the server. For example, if the API call is successful the application developer, in addition to the data requested, will get a 200 code with the OK description. The codes described in this API specification example are some of those HTTP status codes defined by RFC7231 28 HTTP status codes are the common codes used in REST APIs.

\subsubsection{API implementation activity}

This activity refers to building the API functionality according to the API design and specification previously defined. To this aim, it will be necessary to generate the serverside logic to handle each request and return the appropriate response from the SPARQL endpoint. In addition, if a security mechanism was selected in the API definition, it will be necessary to set up the behaviour of the API based on the user executing the request. Figure 4.4 illustrates the interactions that can be done between the client and the endpoint. As depicted in this figure, the API acts as an intermediate layer that allows clients to make requests and these requests are processed by the server to get the responses from the endpoint, and return these responses to the client.

Using IDLs for the API specification gives the added value of inheriting an ecosystem of tooling that can reduce the cost of implementing the API. As most of the common

\footnotetext{
${ }^{28}$ https://datatracker.ietf.org/doc/html/rfc7231\#section-6
} 


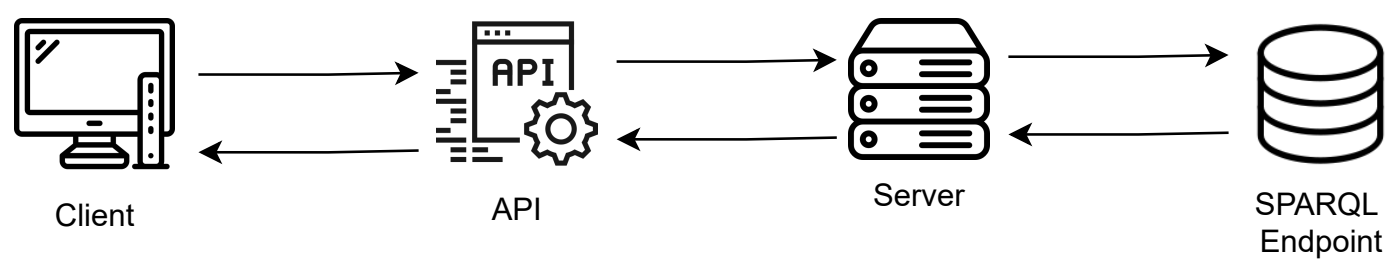

Figure 4.4: Interactions between the client and SPARQL endpoint

API specifications (e.g. OpenAPI) have a big community behind, a set of tools are available for the API implementation. In counterpart, despite IDLs provide users with implementation advantages, they limit the API definitions because users can only use the options available in the language's syntax. Whatever technology or programming language is selected to implement the API, it should make it easy to build and maintain the API.

Following our example above, suppose we develop the server-side functionality to handle the API calls and provide the expected responses. This functionality will include the execution of the SPARQL queries according to each API call, the serialization of the results to deliver them in a developer-friendly format (e.g. JSON), and the configuration of messages to be retrieved (according to the status codes) when executing requests.

\subsubsection{API validation activity}

This activity is carried out jointly by the ontology engineer and application developer, and it is concerned with testing whether the API calls perform the requested behaviour. If this validation fails it will be necessary to go back to the previous activities to review and refine the design, specification or implementation of the API. In our method, the expected behaviour of the API is mainly determined by the competency questions and their answers. However, other ontology artefacts can be used for the API validation, such as use cases and user stories proposed in some methodologies as part of the specification activity, commonly before starting with competency questions. Therefore, the API validation can also be aimed at testing whether the API provides the required functionality and, as a result, allows obtaining the necessary data to satisfy the requirements from these proposed use cases or user stories. Finally, it is worth mentioning that, despite in our method the validation is focused on testing whether the API delivers the data to solve the competency questions, there are other validations that are commonly executed to test any API regarding to, for example, security mechanisms, 
usability, performance and load, among others.

Following our above example, suppose that the server we implemented in the previous activity is up, and that we develop a test script that acts as client to make API calls against this server. Figure 4.5 illustrates the validation example of the API that allows solving the competency question ESCOM1, and presents the interactions between the client, server, and SPARQL endpoint. In the first interaction, client executes the call GET /local-businesses/10003602. Then, the server receives this GET API call which requests data of local business with identifier 10003602, and executes the corresponding SPARQL query that was previously defined for ESCOM1 in Listing 4.1. Next, the SPARQL endpoint returns the attributes and values of the local business. For readability reasons, Figure 4.5 shows few attributes and values delivered by the endpoint and represented in Turtle format. In addition, the prefix escom represents the URI of the local business census ontology, and the attribute names are provided in Spanish since they are defined in the ontology in such language. Finally, the server gets data from the endpoint, formats data in the required serialization (e.g JSON as depicted in this figure), and gets back the response to the client. In our validation example, the response contains the expected data as it was defined in the corresponding output column from the specification example presented in Table 4.6 .

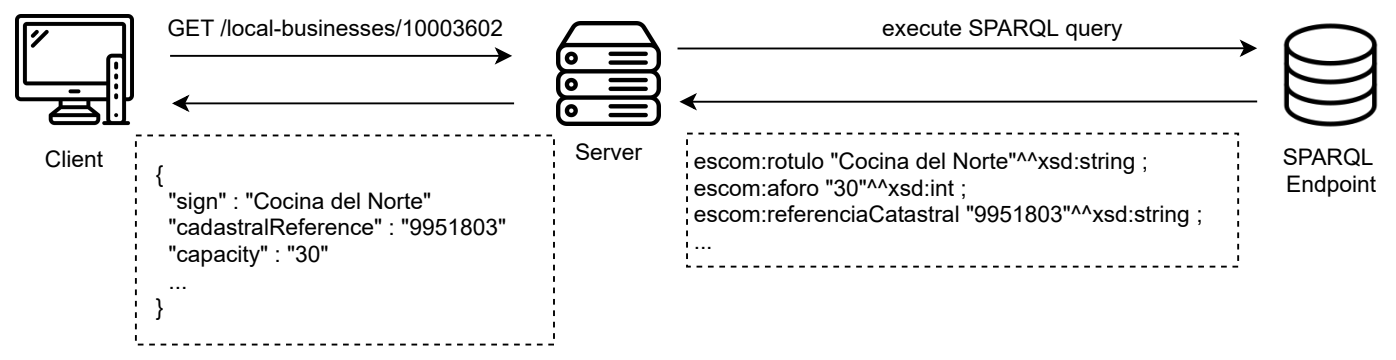

Figure 4.5: API validation by executing an API call to the server.

\subsubsection{API deployment activity}

This last activity aims to make the API on-line available for use. In addition, as in the case of ontologies, in this activity it is worth providing access to documentation to help developers to learn and understand how the API works. Therefore, the API specification, represented in a human-readable format, should be also made available. Finally, it is advisable to make the API findable on the Web to ease its promotion and discoverability. To this end, ontology engineers may publish the API into registries such 
as Programmable Wel29 (for any API or Web service), BioCatalogut 30 (specifically for life science data), or smartAP ${ }^{31}$ (Zaveri et al. 2017) (which requires a OAS extended specification to define key metadata of the API).

Following our above example, suppose we put the server we have implemented online, make the API specification we have defined available on the Web via the URL http://api .example.com, and register the API in the Programmable Web registry.

\subsection{Supporting the API generation process with OATAPI}

Generating an API manually following the steps identified in the previous section can be a tedious, time-consuming, and error-prone task. Therefore, it is advisable to provide ontology engineers and application developers with a technological support that assists them in the API generation process. This section presents OATAPI (Ontology Artefacts to API), a tool developed to automate the execution of some subtasks of the method presented in Section 4.1 .

\subsubsection{OATAPI's overview}

OATAPI is a tool which takes a set of competency questions and the ontology serialization (which fulfills these requirements) and generates the API paths and SPARQL queries that allow getting data to solve these questions. Therefore, OATAPI allows automating the subtasks related to the competency question analysis task from the method for API generation proposed in this chapter. The tool was developed in Python and the code is available in GitHub 32 under Apache License 2.0 33

This tool generates API paths according to the REST paradigm. The decision on using this paradigm was made because REST allows to directly use SPARQL queries to access data that is hosted in traditional SPARQL endpoints. In counterpart, GraphQL requires defining the structure of desired data and having to do specific queries to access such data. Moreover, extra work is required to adopt GraphQL for KG data access, for example, data must be hosted in specialized endpoints (e.g. Comunica engine (Taelman et al. 2018b), or TopBraid (TopQuadrant, 2001)), or additional query languages are required to adapt SPARQL to GraphQL queries (e.g. GraphQL-LD (Taelman et al., 2018c), or HyperGraphQL (Semantic Integration Ltd., 2018)). In

\footnotetext{
$\sqrt[29]{\text { https://www.programmableweb.com }}$

30 https://www.biocatalogue.org

${ }^{31}$ http://smart-api.info/registry

32 https://github.com/paoespinozarias/oatapi

33 https://www.apache.org/licenses/LICENSE-2.0
} 
addition, the APIs collected in the Field Research Work (described in Section 3.7) follow the REST paradigm. Therefore, in the evaluation part of this thesis, it will be necessary to automatically generate REST APIs to compare them with those collected from the Field Research Work.

\subsubsection{Analysis of competency questions}

In this module, OATAPI takes as input the competency questions and the ontology serialization resulting from the ontology development process, and performs the operations detection, ontology elements identification, and query generation subtasks. To automate the analysis, the Spacy ${ }^{34}$ and Owlready2 libraries ${ }^{35}$ have been used. The following list provides general details on the steps that allows OATAPI provide support for the competency question analysis subtasks. The described steps are also formalized in Algorithm 1.

- Competency questions breakdown. It consists of splitting the CQ into pieces to be able to analyze each of them. Then, each piece is tagged according to its respective part-of-speech and dependency tree labels. Each piece will consider only the base form of the terms to perform the analysis described in the next subtasks.

- Operations detection. It identifies what pieces from the previous subtask match terms that denote operations. To that end, the algortihm compares each piece with the terms presented in Table 4.1 .

- Ontology elements identification. It includes the following steps to get the elements:

1. Load the ontology elements into a directed multigraph. To do that, the ontology classes are considered nodes and the object properties as edges. Therefore, OATAPI requires that the object properties contain domain and range restrictions in such a manner that edges contain information about the node from which it originates and to which node it is going.

2. Take each CQ's piece and verify whether it matches the label of ontology elements. If the ontology does not contain labels, the algorithm verifies the URI identifier fragment of the ontology elements (except in case that the ontology follows an opaque URIs strategy).

\footnotetext{
${ }^{34}$ https://spacy.io

35 https://owlready 2 .readthedocs.io
} 
- API paths generation. It regards to the path generation and includes the following steps:

1. Search for coincidences between the ontology elements identified and the nodes from the multigraph.

2. Obtain the shortest path between the nodes found.

3. Take the nodes resulting from the previous step and generate the API path. To this end, OATAPI considers the order of the nodes to build the path. In addition, the tool follows the resource naming practices for REST APIs adapted to the ontology elements as summarized in Annex. A.

- Query generation. It consists of generating SPARQL queries taking as input the nodes detected in the shortest path and the operations identified in the previous steps. The result of this step is a set of SPARQL CONSTRUCT queries.

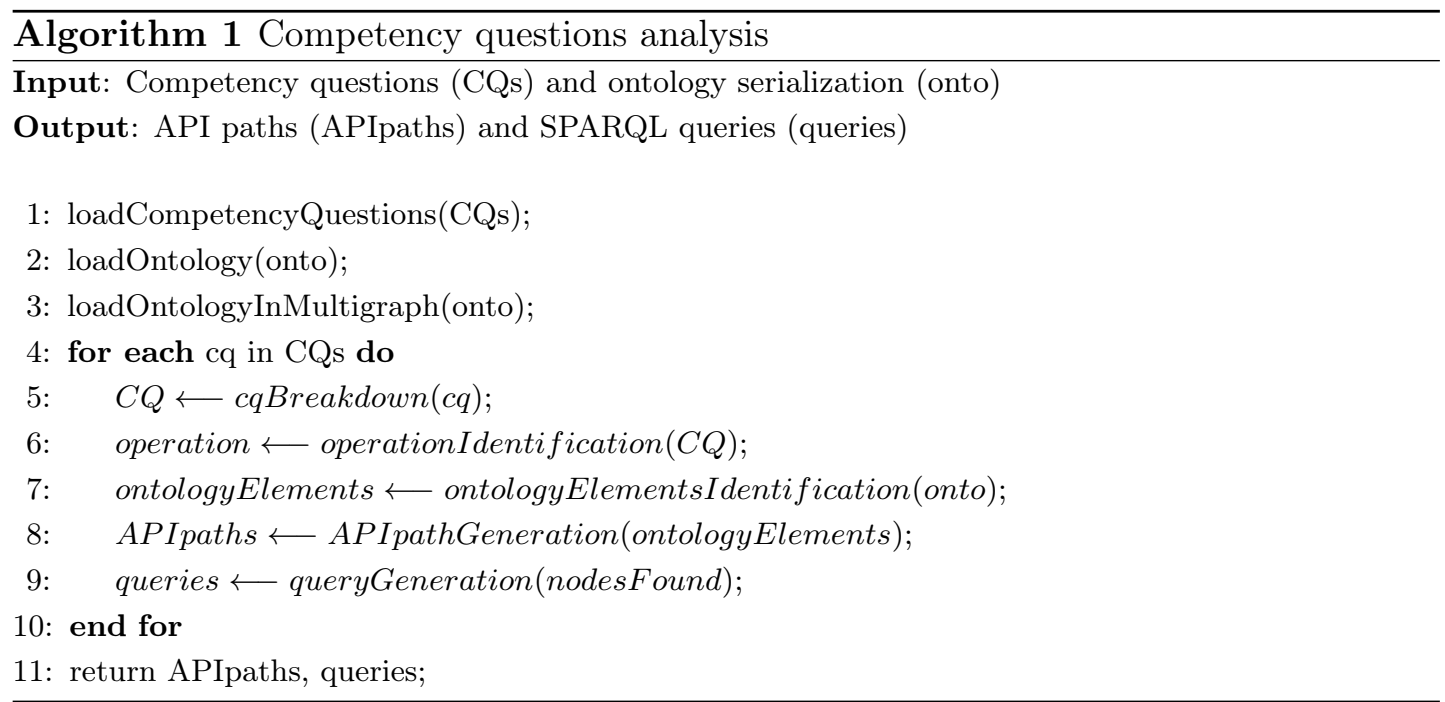

It is worth mentioning that, for readability purposes, the steps described in the API paths generation part describe a common case when the search for coincidences between the ontology elements and the nodes retrieves coincident nodes. However, there are other cases where only one node is detected, where an edge matches with an object property, or both. In such cases the strategy adopted is different because it is not possible to obtain the shortest path between nodes. Therefore, when a node and an edge are found the algorithm searches for the neighbour nodes and verifies whether the 
edge found relates them, if so the algorithm generates the path. In addition, when only an edge is found the algorithm gets the origin and target nodes, and builds the path. Finally, if only a node is found the algorithm automatically generates the path.

In addition, the queries generation step was previously described according to the common case where a shortest path is found. In this case the query structure will be built depending on the nodes and edges found in the shortest path. However, the query structure will change when other cases occur, for example, when only one or two nodes are found. These additional cases are also handled by the algorithm to generate the appropriate queries.

\subsubsection{Complementary support for the API generation activities}

OATAPI allows automating the main subtasks of the API Design activity of the method proposed. However, to carry out some of the remaining activities and tasks there are alternatives to support ontology engineers and application developers to execute them.

\subsubsection{API specification}

In regards to the description of the API design, the OpenAPI Specification (presented in Section 2.3.1) can be used. To facilitate the APIs description, ontology engineers and application developers can reuse the mapping between OWL to OAS ${ }^{36}$ Espinoza-Arias et al. 2020b) that was initially developed for OBA (presented in Subsection 2.3.2.13). This mapping allows translating the ontology elements to the schemas needed for each API path and the common ontology metadata to the corresponding OAS definitions.

After the design activity execution, OATAPIs identified operations and ontology elements, and generated the paths required for the API. With this outputs, the corresponding OpenAPI specification can be manually generated by following the details described in the mapping previously introduced. For example, Listing 4.2 shows the path generated to Terrace in the API. This Path Item defines a GET operation, including a successful response (represented with the 200 HTTP status code ${ }^{37}$ ) which delivers a Terrace schema. The OAS snippet is presented as a YAML file.

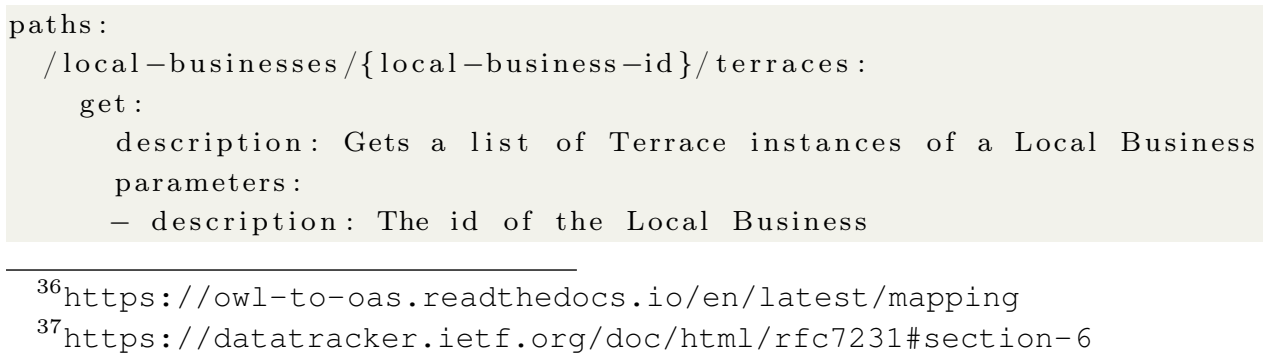

$3 6 \longdiv { \text { https://owl-to-oas.readthedocs.io/en/latest/mapping } }$

37 https://datatracker.ietf.org/doc/html/rfc7231\#section-6 


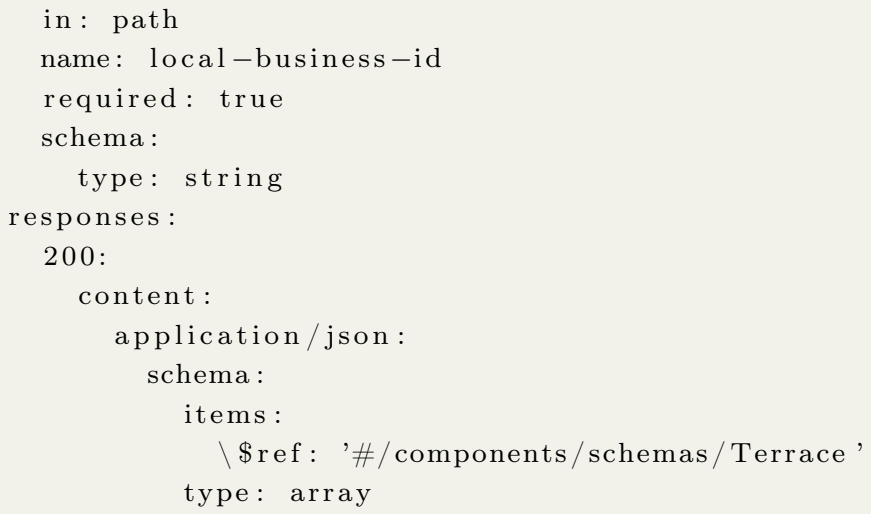

Listing 4.2: OAS snippet of the Path Item Object generated from the operation and ontology elements detection

In addition, Listing 4.3 shows the OAS definitions corresponding to the Terrace class that is the resource required in the API call. The complete OAS file is available in the OATAPIs GitHub repository ${ }^{38}$

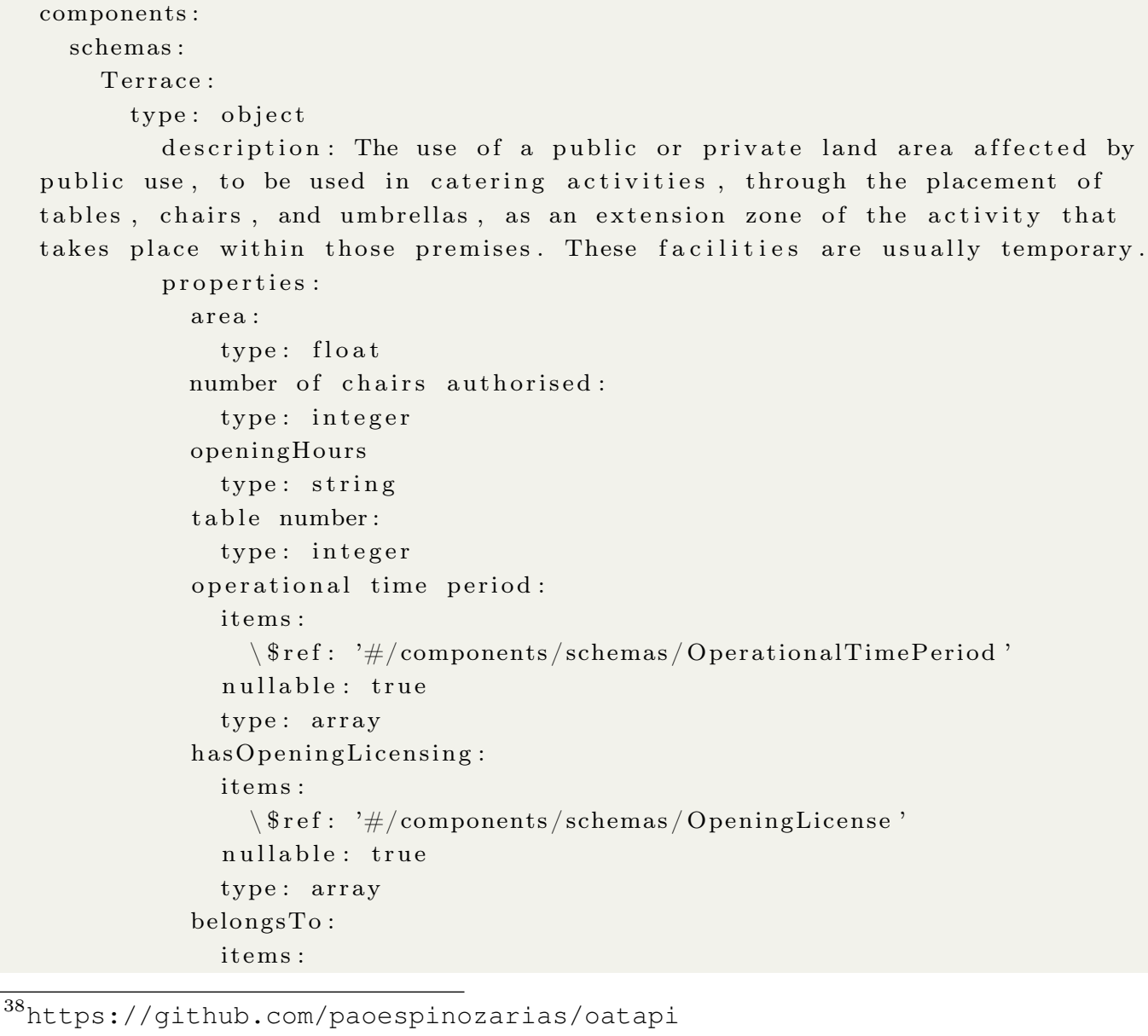




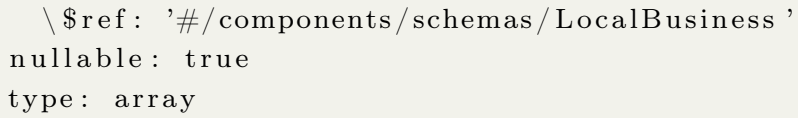

Listing 4.3: OAS snippet of the Component object that includes the Terrace schema

\subsubsection{API implementation and validation}

As for the implementation activity, the OBA framework can be reused to built the server-side logic required for the API functionality. This framework, which has been recently developed and adopted by the community, allows generating generic APIs and queries for the elements of the ontology serialization. However, it also allows adding custom queries 39 for those cases where the generic queries it provides do not cover all the target queries. Therefore, this functionality can be used to configure OBA with the OpenAPI specification manually generated, and the SPARQL CONSTRUCT queries automatically generated by OATAPI.

Regarding the validation activity, OBA also allows ontology engineers and application developers to test, throughout the interactive documentation generated in the previous activity, what are the resources and operations implemented and the responses retrieved after the operations execution. Thanks to this manual validation, the API can be refined if errors or improvements are needed before to deploy it.

Finally, it is worth mentioning that OBA allows configuring a basic authentication mechanism to control access to data. This mechanism is implemented by HTTP requests that include the username and password. However, the user management must be handled by the authentication provider as it is out of the scope of OBA.

\footnotetext{
${ }^{39}$ https://oba.readthedocs.io/en/latest/adding_custom_queries
} 


\section{Chapter 5}

\section{Evaluation}

This chapter describes the evaluation of the research hypotheses proposed in this thesis. More specifically, we evaluate the hypotheses H2 and H3 presented in Section 3.4. First, we evaluate whether API paths automatically generated are indistinguishable from those manually generated ones (Section 5.1). Next, we evaluate the similarity between API paths manually and automatically generated (Section 5.2).

\subsection{Evaluation of indistinguishable API paths}

This section describes the evaluation carried out to determine whether the API paths automatically generated with OATAPI (the tool described in Section 4.2 are indistinguishable from those generated by application developers in real use cases. The following hypothesis was stated already in Section 3.4 .

H2. It is possible to automatically generate APIs (based on ontology artefacts) that are indistinguishable from the APIs that would be defined by application developers in real use cases.

\subsubsection{Experimental design}

The goal of this experiment is to test by means of a questionnaire what is the perception of the participants regarding several API paths that were generated by OATAPI and by developers in real use cases. To that end, given a competency question of a specific ontology domain each participant has to answer whether the given API paths have been manually or automatically generated, or is not possible to determine that.

For the purpose of this evaluation, a testbed was generated. Initially, it contained the following two elements: 1) a set of competency questions and ontologies from dif- 
ferent domains, and 2) the API paths generated by developers in real use cases. The elements of the testbed were gathered during the field research work phase of our research (described in 3.7). More precisely, some elements were gathered from the Ciudades Abiertas (Open Cities (OC)) ${ }^{40}$ a project where several Spanish cities have been working together to create a shared set of ontologies to provide homogeneous data access in their open data portals and APIs. In addition, other elements were gathered from the Zaragoza city council (ZGZ), such elements have been generated as part of the Zaragoza's open-data-by-default policy. One of the main assets of this policy is the Zaragoza's Knowledge Graph, which clusters the Zaragoza's open data, which are described with ontologies, in order to exploit the knowledge of the city (Espinoza-Arias et al. 2020a). In both cases (OC and ZGZ) an attempt has been made to automatically generate the APIs; however, these APIs are not based on or generated from the competency questions or the ontology serialization. Finally, taking as input the CQs and ontologies collected in the testbed, OATAPI was executed to automatically generate their API paths. Then, such paths were included in the testbed. The list of elements used in this experiment is shown in Table 5.1 .

It is worth mentioning that the CQs and API paths from OC and ZGZ are originally written in Spanish. Therefore, these CQs and API paths were translated into English to generate the API paths with OATAPI, and to be able to compare these API paths with the translated API paths from these real uses cases. The script to generate the API paths, and the complete testbed are available in the evaluation folder from the OATAPI's GitHub repository 41 This testbed contains the original and translated CQs and API paths, and the API paths generated with OATAPI. In addition, it includes the ontology IRI, and the URL of the source of the APIs and CQs. Finally, Table 5.1 presents the translated CQs and API paths from the real use cases that were evaluated in this experiment.

\footnotetext{
40 https://ciudadesabiertas.es/

${ }^{41}$ https://github.com/paoespinozarias/oatapis/tree/main/Evaluation
} 


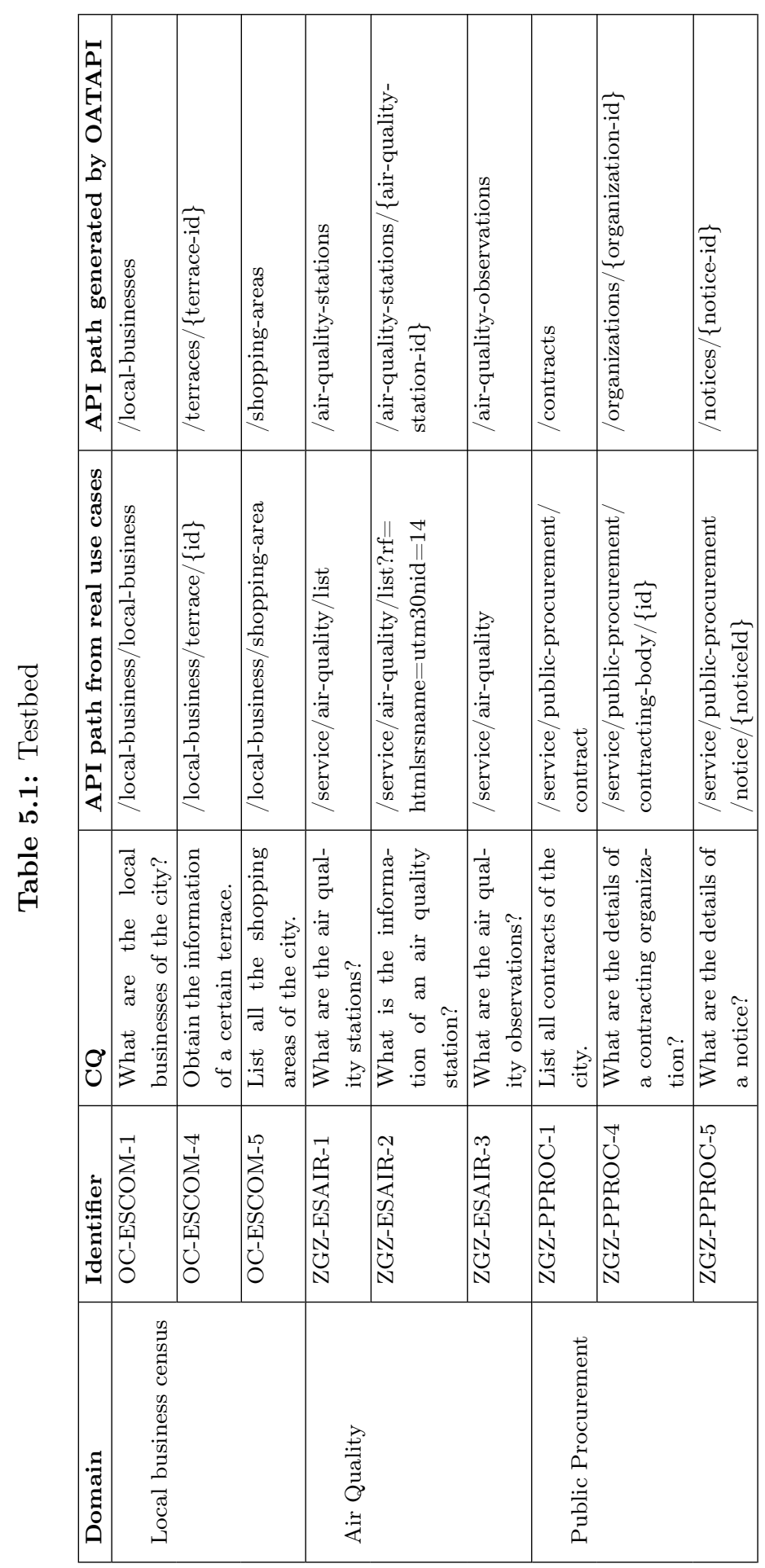


With the aim of comparing the API paths from the testbed, an online questionnaire was created for the participants of the experiment. Such questionnaire collected data from participants and their results regarding the way the API paths were generated and which ones they would prefer to use. First, the questionnaire asked participants to add information about their background, including expertise in WebServices, APIs, and semantic technologies. Then, the questionnaire asked participants about the expected answer after the execution of a GET operation over some REST API calls.

Afterwards, the participants were asked about API paths gathered in the testbed for each CQ from three different domains. The following information had to be added by each participant for each couple of API paths:

- The way API paths were generated: (1) The 1st is generated automatically and the 2nd is generated manually; (2) The 2nd is generated automatically and the 1st is generated manually; (3) Both are generated manually; (4) Both are automatically generated; or (5) It is not possible to determine how they were generated (Indistinguishable).

- From the API paths presented, the API path participants would prefer to use.

The questionnaire is included in Annex $[$ and the results obtained are available in the evaluation folder from the OATAPI's GitHub repository 42

\subsubsection{Results and discussion}

In this evaluation, 20 participants were involved. The following subsections provides details on the results obtained from the questionnaire.

\subsubsection{Background analysis}

Among participants there was a good variety of very familiar (have designed, implemented, and used certain technology), familiar (have only used certain technology), and non familiar with WebServices, REST APIs, and GraphQL. Table 5.2 summarises the familiarity of participants in regards to technologies. The results presented in this table reveals that the majority of participants are very familiar with Web Services and REST APIs. However, the majority of them are not familiar with GraphQL.

\footnotetext{
${ }^{42}$ https://github.com/paoespinozarias/oatapis/tree/main/Evaluation
} 
Table 5.2: Familiarity of the participants with the technologies

\begin{tabular}{|l|c|c|c|}
\hline Technology & Very familiar & Familiar & Not familiar \\
\hline Web Services & 14 & 4 & 2 \\
\hline REST APIs & 16 & 2 & 2 \\
\hline GraphQL & 2 & 3 & 15 \\
\hline
\end{tabular}

Continuing with the analysis of the background of the participants, Figure 5.1 shows the obtained results with regards to the number of Web APIs that they have developed. From this figure it can be observed that, regardless the majority of participants argued that they are most familiar with Web Services and REST APIs, the majority of participants have only developed between 1 and 5 Web APIs.

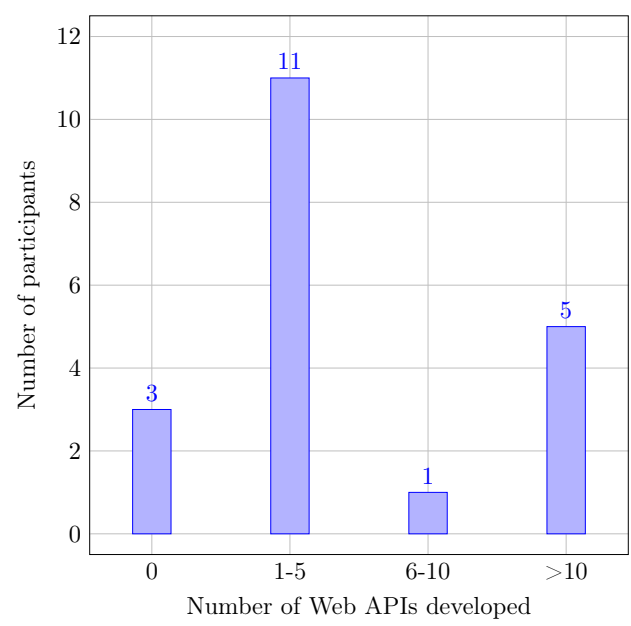

Figure 5.1: Number of Web APIs developed per participant

To end the analysis of the background, Figure 5.2 summarizes the results obtained with regards to the familiarity of participants with semantic technologies, and which ones they are used to. This figure shows that the majority of participants (85\%) are familiar with semantic technologies. As a result of this familiarity, participants fulfilled (in a free text box) what are the technologies they are used to. Therefore, the names provided by participants were categorized as semantic specifications, tools, and serializations. From this figure it can be observed that, the majority of participants mentioned that they are used to SPARQL, OWL, and RDF specifications. However, a few number of mentions were done for tools and serializations. Regarding these tools, participants mentioned some that provide support to ontology engineering activities (Protégé and OnToology), to handle RDF data in applications (Jena), and to man- 
age triples in a store (Virtuoso). Finally, participants mentioned two human-friendly serialization formats (Turtle and JSON-LD).

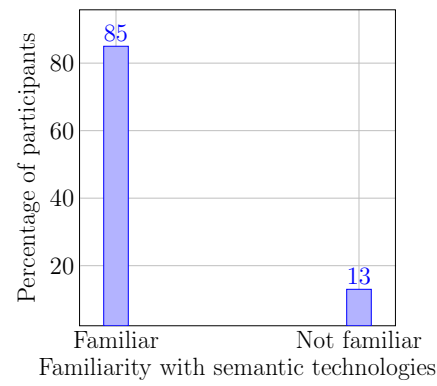

(a) Background in semantic technologies

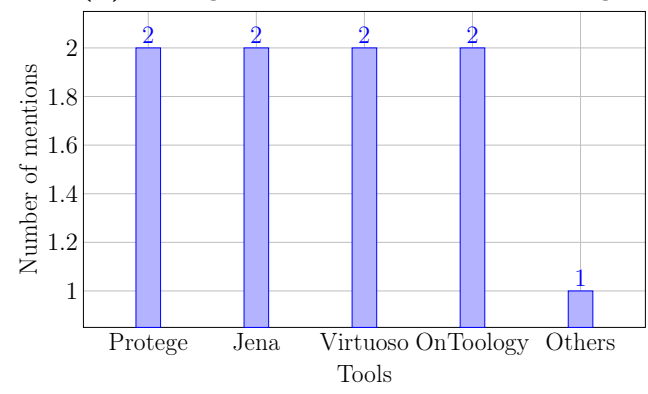

(c) Semantic tools

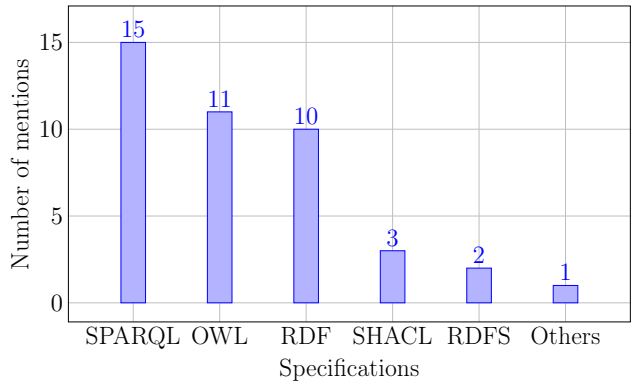

(b) Semantic specifications

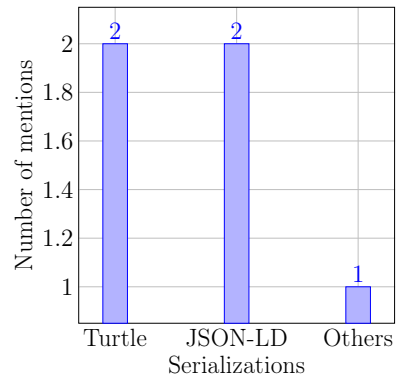

(d) Semantic serializations

Figure 5.2: Participants' background in semantic technologies, and specifications, tools, and serializations they are used to.

\subsubsection{REST APIs behaviour analysis}

As for specific questions regarding REST APIs, this part of the evaluation allows to know whether the participants recognize the structure of an API path and what resource(s) would be expected back after the execution of the GET operation on this path. For example, a participant that knows how an API works would know that the API call GET / resources (e.g. GET / cities) should get back a list of resources (e.g. a list of cities such as for example Madrid, Paris, London, etc.). Figure 5.3 shows the results obtained from the questionnaire.

From this figure it can be observed that the majority of participants selected the correct expected answer for the execution of the corresponding API call. However, few participants failed to select the answer regarding the API path/results/\{resources-id\}; they selected as answer a list of resources, and a list of resources matching the parameters. These results lets us know that the structure of the paths that the participants 
will see in the next part of the evaluation are familiar to most of them.

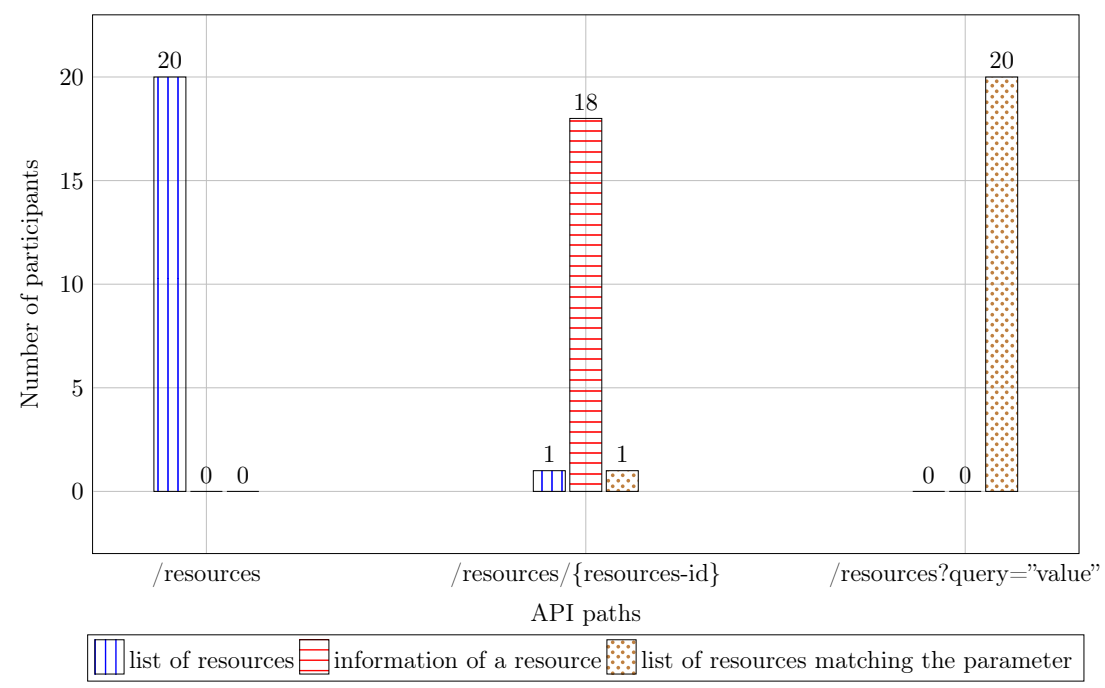

Figure 5.3: Expected answers after executing a GET operation on a API path.

\subsubsection{API paths based on ontology artefacts analysis}

Table 5.3 summarizes the results obtained from the evaluation of the API paths presented to the participants. In this table the "1st" term stands for the API paths generated by developers from real uses cases, and the "2nd" term stands for the API paths automatically generated by OATAPI. From this table it can be observed that, in general, in regards to the way in which APIs were generated the participants mostly selected the first and the last option. The first option states that the 1st API was generated automatically and the 2 nd was generated manually. The last option argues that is not possible to determine how they were generated (Indistinguishable). 


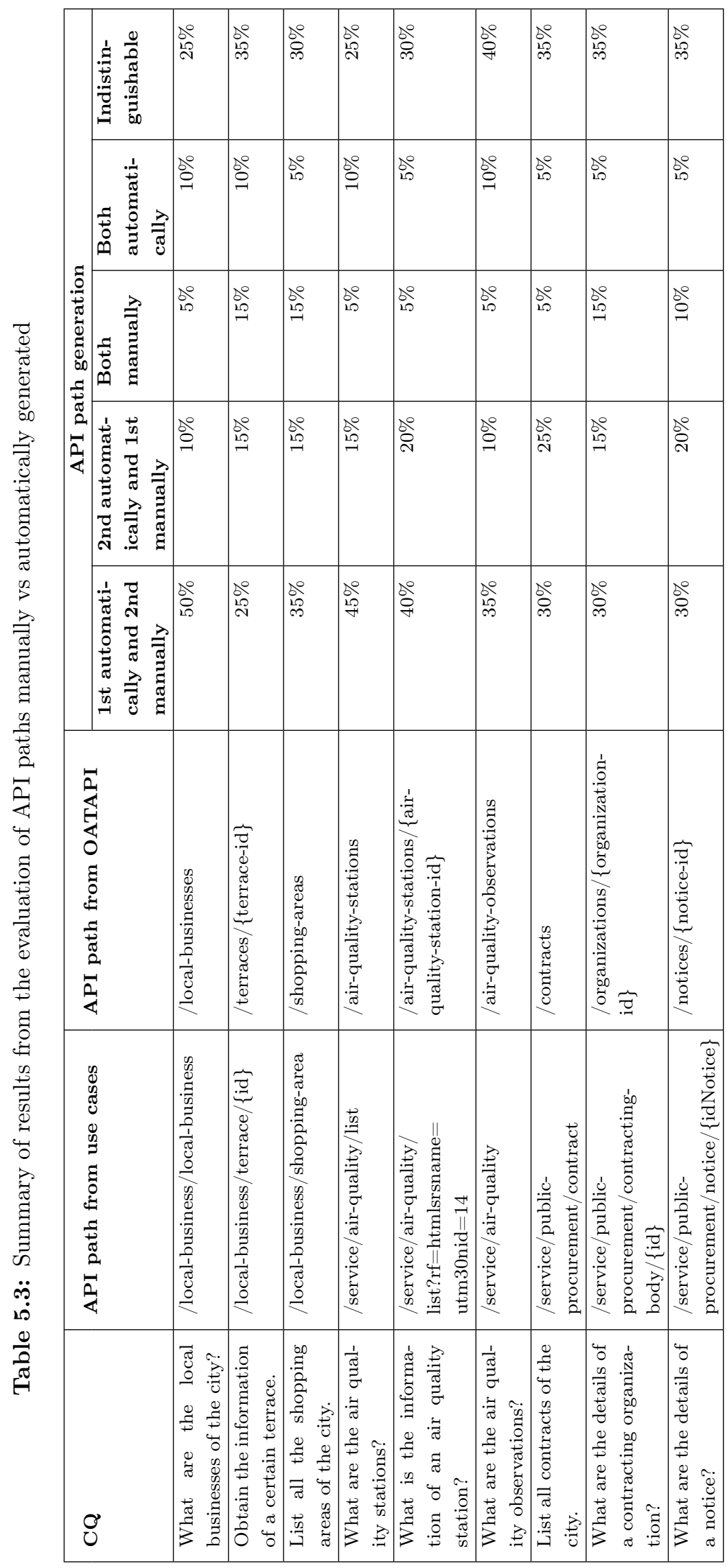


On the one side, the highest percentage of participants (50\%) who choose the first option are those who argued that API path/local-business/local-business was automatically generated and API path/local-businesses was manually generated. This selection could be influenced by the fact that the/local-business/local-business path includes the resource name twice. For the same API paths, a considerable percentage of participants $(25 \%)$ were not able to distinguish how they were generated.

On the other side, the highest percentage of participants (40\%) who selected the last option are those who examined /service/air-quality and /air-quality-observations paths. In this case participants were not able to distinguish how API paths were generated, but also a big percentage of participants $(35 \%)$ believed that the 1st and 2nd paths were automatically and manually generated respectively.

Finally, Table 5.4 summarizes the results obtained about the preferences of participants in regard to which API path they would prefer to use. From this table it can be observed that in most of the cases participants prefer those API paths automatically generated by OATAPI. In addition, the API path generated by developers with the lowest percentage of preference $(5 \%)$ is the one that was explained in the previous table, i.e the one that duplicates the resource name (/local-business/local-business).

\subsubsection{Conclusions of the experiment}

With respect to the hypothesis $\mathrm{H} 2$, it can be concluded that it is possible to automatically generate API paths that are indistinguishable from those manually generated. Moreover, it can be concluded that OATAPI allows building automatically API paths that had a significant higher percentage of preference for participants in regard to those generated by developers in real use cases. Finally, it can be concluded that these automatically generated paths could positively impact the real world. Application developers will be able to recognize the structure and behavior of these API paths which will allow them to consume data from KGs for use it in their applications. 


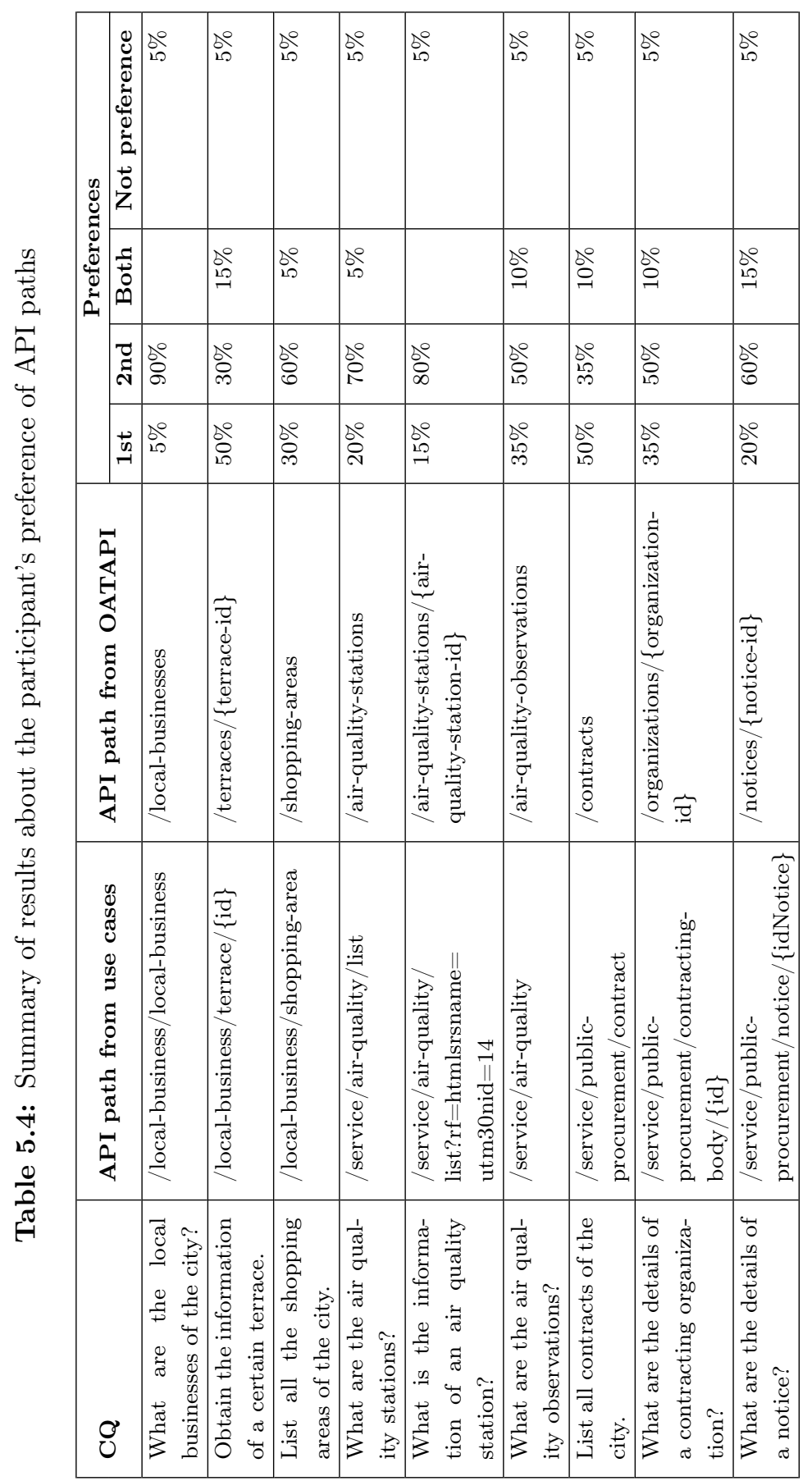




\subsection{Evaluation of similarity between API paths}

Chapter 4 presented the method to generate API paths from ontology artefacts (detailed in Section 4.1) and the technological support provided by OATAPI to support the automatic API generation (presented in Section 4.2). This section describes the evaluation carried out to determine whether OATAPI is able to generate APIs that are similar to those manually defined ones. The following hypothesis was made for this evaluation:

H3. It is possible to automatically generate APIs (based on ontology artefacts) that are similar to the APIs defined manually according to our proposed method.

\subsubsection{Experimental design}

This evaluation experiment is composed of three stages: 1) manual generation of API paths for a set of competency questions and ontologies, 2) automatic generation of API paths for the set of competency questions and ontologies used in the previous stage, and 3) similarity comparison between API paths obtained from the first and second stages. In the following subsections we provide further details on each stage.

\subsubsection{Manual generation of API paths}

Regarding the first stage, we generate a corpus that contains a set of CQs and their API paths. To generate such corpus, we collected two ontology artefacts: a) the ontology serialization, and b) a set of its competency questions written in natural language. To this end, we collected some ontology serializations and competency questions from ontologies of the state of the art like the VideoGame - VGO (Parkkila et al., 2017), Public Procurement - PPROC (Muñoz-Soro et al., 2016), Software - SWO (Malone et al., 2014), and SAREF for Environment - SAREF4ENVI (ETSI, 2020). We also took some of the ontology artefacts from ontologies of Open Cities (Ciudades Abiertas) ${ }^{43}$ and the Zaragoza city council (Espinoza-Arias et al. 2020a) projects previously described in the experiment from the evaluation of indistinguishable API paths (Section 5.1).

Then, we manually performed the ontology elements identification subtask described in Subsection 4.1.1.2. To this end, we analyzed each CQ and the ontology serialization to identify the ontology concepts that must be included in the API path. Next, we generated the API paths according to the rules for naming REST APIs based on ontology artefacts provided in Annex A. Table 5.5 presents an excerpt of the corpus generated

$\sqrt[43]{\text { https: / / ciudadesabiertas.es/ }}$ 
for the CQs of the VideoGame ontology. This table shows the competency question identifier, the competency question itself, and the API path generated manually.

Table 5.5: Excerpt of the corpus of the VideoGame ontology CQs.

\begin{tabular}{|l|l|l|}
\hline Identifier & CQ & Expected API path \\
\hline VGO-1 & $\begin{array}{l}\text { What is the username of the } \\
\text { player? }\end{array}$ & $/$ players $/$ player-id $\}$ \\
\hline VGO-2 & $\begin{array}{l}\text { Who are the friends of the } \\
\text { player? }\end{array}$ & $/$ players $/\{$ player-id $\} /$ players \\
\hline VGO-5 & $\begin{array}{l}\text { What are the achievements of } \\
\text { my friends? }\end{array}$ & $/$ players $/\{$ player-id $\} /$ players $/$ achievements \\
\hline VGO-8 & $\begin{array}{l}\text { What achievements does a game } \\
\text { have? }\end{array}$ & $/$ games $/\{$ game-id $\} /$ achievements \\
\hline VGO-14 & Who is the creator of the game? & $/$ games $/\{$ game-id $\} /$ agents \\
\hline
\end{tabular}

It is worth mentioning that in our evaluation we only considered questions and we excluded those competency questions that are defined as facts because they do not have an expected answer. In addition, it is important to mention that in the corpus we described some API paths as "undefined" because the corresponding CQs:

- Are ambiguous. For example, "What are the games similar to this one?". The term "similar" could be interpreted in different ways. Therefore, its resolution is subjective.

- Contain elements that are not defined in the ontology (Restriction R4 described in Section 3.5).

- Contain a synonym. For example, "What abilities does an item have?". The term "abilities" is not defined in the ontology. We could interpret this term as "features" which is a concept defined in the ontology and it is defined as an ability or characteristic. However, OATAPI is not designed to make this kind of analysis.

- Contain an instance. For example, "How much damage does a weapon deal?". The term "weapon" does not exist in the ontology. However this term could be interpreted as a specific item of a game, therefore as an instance of the item class. In addition, this CQ is ambiguous because we are not able to determine what "damage" means.

- Are complex. For example, "If a player is given free item in the game, how likely are they to make an in-app purchase?". This CQ aims to exploit knowledge 
generated by the information gathered from several instance values. Therefore, we cannot define manually an API path according to the rules defined in our method, and OATAPI cannot generate it automatically either.

- Contain terms that correspond to properties in the ontology, but these properties do not have domain and range (Restriction R7 described in Section 3.5). For example, "Which properties can be measured by a photometer?". In this CQ (from the SAREF4ENVI ontology) the object property detected to get the properties is measuresProperty. However, such property does not define Photometer as domain, and LightProperty as range. Despite the class Photometer includes as restriction that the property measuresProperty has some LightPropery, the restrictions made in classes are not considered in our proposed method and in OATAPI neither.

At the end of this stage, a total number of 8 ontology serializations and 228 CQs were manually analyzed. Table 5.6 provides a summary of the ontologies together with the CQs analyzed, and the CQs that are valid to manually generate the expected API paths.

Table 5.6: Summary of ontologies, CQs manually analyzed, and valid CQs.

\begin{tabular}{|l|c|c|}
\hline Ontology & CQs manually analyzed & Valid CQs \\
\hline VGO & 67 & 23 \\
\hline ESCOM & 19 & 8 \\
\hline SWO & 91 & 3 \\
\hline SAREF4ENVI & 15 & 2 \\
\hline NOISE & 7 & 3 \\
\hline PPROC & 17 & 5 \\
\hline ESAIR & 3 & 3 \\
\hline ESBICI & 9 & 4 \\
\hline Total & $\mathbf{2 2 8}$ & $\mathbf{5 1}$ \\
\hline
\end{tabular}

\subsubsection{Automatic generation of API paths}

As for the second stage, we took as input the ontology serialization and the set of competency questions collected in the first stage and we executed OATAPI. It is worth mentioning that in this stage we did not consider those CQs with API paths described as "undefined". As a result of OATAPI's execution, we obtained a set of 51 API paths generated automatically for the CQs that did not have undefined API paths. Table 5.7 summarizes the API paths generated per ontology. 
Table 5.7: API paths automatically generated with OATAPI

\begin{tabular}{|l|c|}
\hline Ontology & API paths generated \\
\hline VGO & 23 \\
\hline ESCOM & 8 \\
\hline SWO & 3 \\
\hline SAREF4ENVI & 2 \\
\hline NOISE & 3 \\
\hline PPROC & 5 \\
\hline ESAIR & 3 \\
\hline ESBICI & 4 \\
\hline Total & $\mathbf{5 1}$ \\
\hline
\end{tabular}

\subsubsection{Similarity comparison}

Regarding the third stage, we use the ROUGE (Recall-Oriented Understudy for Gisting Evaluation) metric to compare the similarity between the API paths generated in the two previous stages. ROUGE $(\overline{\operatorname{Lin}}, \overline{2004})$ is a set of metrics aimed to evaluate the similarity between two summaries or translations; one of them created by humans, and the another one generated by machines with natural language processing techniques. Therefore, the objective of this metric fits the evaluation we need to do between the API paths generated by humans (according to our proposed method), and those paths automatically generated with OATAPI, (which applies natural language processing techniques).

ROUGE provides several metrics such as ROUGE-N, ROUGE-L, and ROUGE-S. Each of these metrics performs an evaluation according to certain structure of the summary or translation content. In our evaluation, we use the ROUGE-N metric which allows measuring the number of $\mathrm{N}$ matching tokens/words. More precisely, we use ROUGE-1 to measure the match-rate of one token between the API paths because each API path is provided as a single token. ROUGE metric allows us to calculate the recall, precision, and F1 score between the API paths. In order to calculate ROUGE-1, we use the rouge Python library 44 The script to calculate this metric, the corpus generated in the first stage, the API paths generated in the second stage, and the results of the ROUGE metric are available in the evaluation directory from the OATAPI's GitHub repository. 45 .

\footnotetext{
$4 4 \longdiv { \text { https://pypi.org/project/rouge } }$

45 https://github.com/paoespinozarias/oatapis/tree/main/Evaluation
} 


\subsubsection{Results and discussion}

In the similarity evaluation, 51 API paths manually and automatically generated were compared with the ROUGE metric. Table 5.8 presents the values obtained from this comparison. Note that the values shown in the precision, recall, and F-measure columns represent the sum of the result of each metric value obtained after executing each comparison. These values are round numbers since we obtained 1 or 0 for these metrics after evaluating each couple of paths. This occurred because we evaluate API paths as one token; therefore ROUGE can only determine if there is a full syntactical match or not between the paths evaluated. For example, in this table in the VGO ontology there are 15 machine generated API paths which have a complete syntactic match with the manually generated ones. More precisely, since we obtained 1 for precision and recall for each match, we then have in total 15 for all the matches in precision and recall columns of this ontology, and 15 as the F-measure value which is also the sum of the each single value obtained from the calculus between precision and recall.

Table 5.8: Results obtained from the ROUGE metric evaluation of the API paths manually and automatically generated.

\begin{tabular}{|l|c|c|c|c|}
\hline Ontology & Precision & Recall & F-measure & API paths evaluated \\
\hline VGO & 15 & 15 & 15 & 23 \\
\hline ESCOM & 8 & 8 & 8 & 8 \\
\hline SWO & 0 & 0 & 0 & 3 \\
\hline SAREF4ENVI & 1 & 1 & 1 & 2 \\
\hline NOISE & 3 & 3 & 3 & 3 \\
\hline PPROC & 5 & 5 & 5 & 5 \\
\hline ESAIR & 3 & 3 & 3 & 3 \\
\hline ESBICI & 3 & 3 & 3 & 4 \\
\hline Total & $\mathbf{3 8}$ & $\mathbf{3 8}$ & $\mathbf{3 8}$ & $\mathbf{5 1}$ \\
\hline
\end{tabular}

Figure 5.4 shows the percentage of automatically generated API routes matching manually generated ones 46 This values indicate the amount of API paths that OATAPI was able to generate with regards to the total number of API paths evaluated. The values retrieved give us a measure of the algorithm performance that relies on its ability to generate an API path structure equal to the one generated by a human.

\footnotetext{
${ }^{46}$ For readability the numbers shown in this figure are rounded.
} 


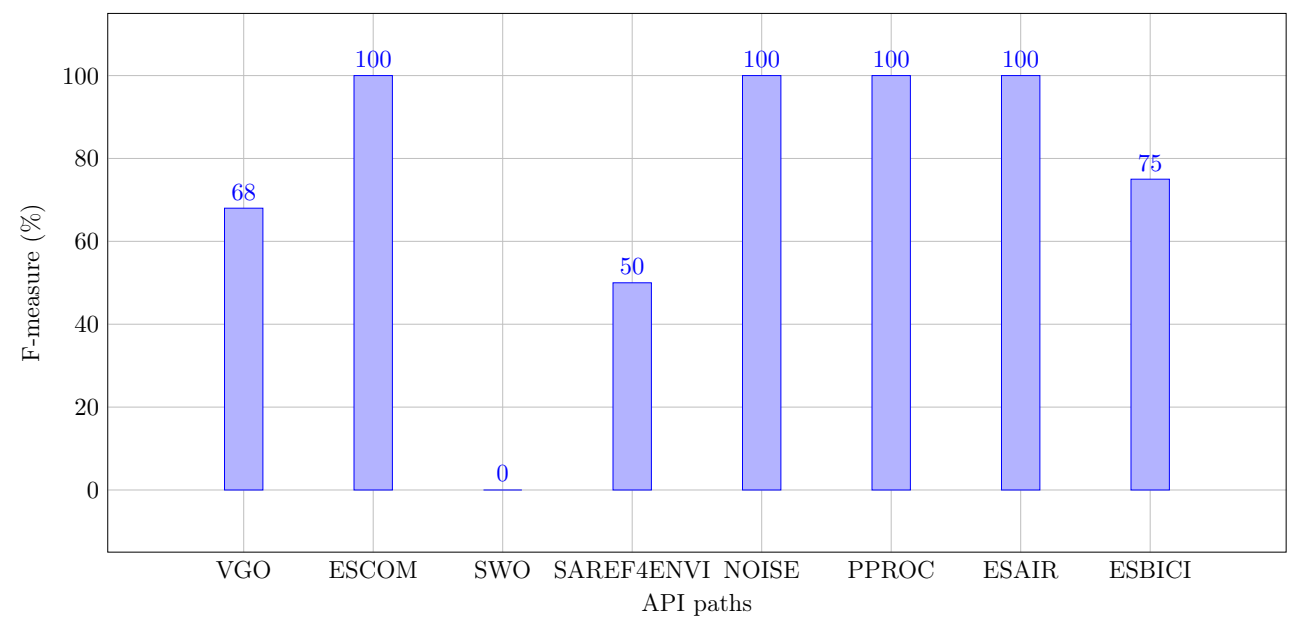

Figure 5.4: F-measure of automatically generated API routes matching manually generated ones.

Regarding correct results, from the previous table it can be observed that OATAPI is able to generate most of the API paths for the CQs and ontologies evaluated. A total number of 38 of 51 API paths were correctly generated in an automatic way. This result corresponds in average to the $72 \%$ of the total of API paths automatically generated.

Concerning incorrect results, the API paths with the lowest percentage of F-measure are those from the SWO, as it can be observed in Figure 5.4. In this case, the automatic generation cannot be correctly done because CQs are too verbose or do not contain the same name of the entity as it is defined in the SWO ontology. Regarding the next low percentage result (50\%), it corresponds to the SAREF4ENVI ontology. In this case, despite only two questions from this ontology were analyzed, OATAPI failed in the generation of one API path because the label of the target concept (TESS) is too long. As for the VGO ontology, the $68 \%$ of API paths were well generated. In this case, the incorrect results occurred because CQs are not enough clear to be translated; i.e. they do not include the same terms that are in the VGO ontology. For example, expressions like "my friend" or "your friend" should be more explicit like "the friend of the player", that would allow OATAPI to generate the API path. Finally, the $75 \%$ of API paths of ESBICI ontology were well generated. In this case, OATAPI failed in the generation of one API path because the CQ ("How many dock stations are operational/non-opperational?") is not precise. It should ask for operational or nonoperational dock stations but not both. 


\subsubsection{Conclusions of the experiment}

With respect to the hypothesis $\mathrm{H} 3$, we can conclude that it is possible to automatically generate similar API paths than those that would be manually generated following the method proposed in Section 4. OATAPI allows building API paths that are identical to those generated manually, as evidenced by the high percentage of matches obtained in the evaluation. However, the automatic API paths generation will depend largely on the conciseness and clarity of the competency questions, and the completeness of the restrictions defined in the ontology. 



\section{Chapter 6}

\section{Conclusions and future work}

This thesis addresses an open research question related to the exploitation of ontologies, that have been built using good ontology engineering practices, to generate APIs based on ontology artefacts created by ontology engineers during the ontology development process (defined in Chapter 3.1). The work in this thesis aims at helping ontology engineers and application developers generate APIs by proposing a method to generate such interfaces from the ontology artefacts. This thesis also intends to provide a technological solution to automate some of the tasks of the proposed method. This chapter summarizes the conclusions regarding the contributions to the state of the art. The rest of the chapter provides an outlook of the current limitations and the future lines of work.

\subsection{Contributions}

The first contribution of this thesis is a method for API generation based on ontology artefacts. This method provides clear details on a set of activities and tasks to generate APIs that allows consuming data from KGs (Section 3.4. H1). This contribution also includes the definition of the ontology exploitation phase as part of the ontology development process, a new phase to be considered in the last stages of ontology development methodologies. This phase encompasses any activity where the ontology must be used after it has been serialized or published. The proposed method is part of this phase as it provides guidelines on how to provide an ontology-based data consumption mechanism through APIs. This contribution also includes a set of rules for naming REST APIs based on ontology artefacts. Such rules allow ontology engineers and application developers to follow a standardized way of defining API paths 
when a REST paradigm strategy has been adopted.

The second contribution of this thesis is OATAPI, a tool for supporting some tasks of the API generation process. This tool takes the ontology serialization and its competency questions to generate the API paths and SPARQL queries required to get data which allows solving these CQs. Therefore, OATAPI allows automating the competency questions analysis task from the API design activity of the method proposed. As shown in Section 5.1. OATAPI generates APIs that are indistinguishable from those defined by humans. These APIs generated automatically are also preferred by users in comparison with those gathered from real use cases. In addition, as shown in Section 5.2, the tool generates APIs similar to those defined manually by humans following the details of the proposed method. This contribution also includes a mapping between $O W L$ and $O A S$ which provides ontology engineers and application developers with a set of guidelines on how to translate ontology elements into the corresponding definitions of the OpenAPI specification for REST APIs.

Based on these contributions, the main conclusions of the thesis are summarized as:

- The adoption of definitions, techniques, and processes taken from the Software Engineering field is a common and helpful practice for Ontology Engineering. Software Engineering is a mature field that has provided solutions to problems that are similar to those from the Ontology Engineering. Therefore, during the last years the Ontology Engineering community has adopted and adapted some of these solutions, as in the case of the method proposed to generate APIs which intends to bridge the gap between application developers and Semantic Web experts.

- The knowledge acquired about the domain by the ontology engineer and the ontology artefacts generated during the ontology development process can support the generation of APIs. Rather than letting application developers generate such interfaces on their own, the involvement of ontology engineers in this process is helpful because they known exactly what is defined in the ontology and the ontology artefacts. From the analysis of the APIs defined only by application developers in real use cases, it can be observed that these interfaces do not have a direct correspondence to the CQs defined in the ontology, and the naming strategy followed in some of these interfaces do not use the names of the ontology elements. Therefore, it makes it difficult to understand by others which resources (based on the model) the interfaces are exposing and what the expected responses are. 
- Making ontology artefacts publicly available is as important as making their implementation available. Despite publishing the intermediate ontology artefacts (CQs, glossary of terms, SPARQL queries, etc.) generated during the ontology development is not mandatory, it is useful for providing more information about the ontology. In this work, significant difficulties to found competency questions were found because mostly ontology papers list few illustrative CQs. This fact, also reported in previous works (Wiśniewski et al., 2019) (FernándezIzquierdo et al., 2019), hindered the analysis of CQs that could be useful to improve the algorithm to generate the API paths and SPARQL queries. Publishing the intermediate ontology artefacts could allow investigating and reusing them to provide new solutions for ontology documentation, formalization, testing, and exploiting.

- The definition of competency questions should be as precise as possible. From the analysis of the CQs found, it can be observed that there are several ambiguous, verbose, and imprecise expressions in these questions. This fact made it difficult to analyze them in regards to the definitions provided in the ontology, and, as a result, limit the APIs definition.

\subsection{Future work}

This section presents some open challenges or problems that have not been addressed in this thesis or that arose as a continuation of the contributions proposed in this work. In regards to the contributions of this thesis, the lines of future work include:

- Improvement of OATAPI functionality. The current version of this tool can be refined to allow addressing its current limitations. To that end, some of the identified future work would be:

- Adopting a more robust approach for the $C Q$ analysis. The natural language analysis performed by the tool relies on the Spacy library, that for ends of a proof of concept was enough to illustrate its functionality. However, to provide better results and to allow analyzing complex CQs, the tool could be improved by adopting robust NLP methods, e.g. supervised learning.For supervised learning, it could reuse the large set of CQs patterns that have been released (Wiśniewski et al., 2021).

- Exploiting inference for ontology element identification. Currently, the tool uses the domain and range restrictions on object data properties to build 
the relationships between nodes in the multigraph, and based on its content provides the matching ontology elements. Therefore, using ontology inference could benefit the detection of elements whose relationships are inherited from the parent classes/properties, but are not explicitly included in the multigraph.

- Enabling synonym detection. CQs are gathered at the early stages of the ontology development; therefore, in some cases they do not include a unified definition for the same concept. To facilitate the detection of the ontology elements, the terms included in the glossary can be reused. In addition, natural language resources that contain synonyms can be reused to improve the ontology elements identification, e.g. BabelNet (Navigli and Ponzetto, 2010).

- Generating the OAS automatically. The tool can be extended to also support the API specification activity of the proposed method. To that end, the mapping defined to translate OWL to OAS can be automated. Therefore, as the tool is able to identify the ontology elements that are required for the API, these elements can be translated into the OAS definitions required for describing the API.

- Annotation of CQs. As mentioned in the previous section 6.1, publishing the CQs gathered for the ontology development is important. Therefore, annotating these queries with the ontology elements detected in their analysis would allow users to have another source of documentation that could help them to understand the ontology. In this manner, if users click on certain term of the CQ they can be redirected to its definition in the human-readable ontology documentation. In addition, including a link in the CQs that allows users to be redirected to its specific API path in the API documentation.

- Extension of OATAPI evaluation. Despite OATAPI has been evaluated in two ways, more evaluations are still needed; therefore, two new evaluations are planned. One of them is testing whether the resulting API allows applications developers to easily implement an application that consumes data from the KG. In this evaluation, the target application could be one that allows implementing the use cases or user stories proposed for the ontology development. The other evaluation is testing OATAPI in real usage scenarios. This last evaluation would allow gathering feedback from real users and refining not only the tool but also the method proposed. 
In regards to open challenges, the new research directions identified include:

- API resource path prediction. Predicting relevant paths based on the data available in a KG could help automatically retrieving the most relevant resources in such KG. To this end, the relevant resources can be detected by calculating e.g., their number of connections, frequency or other metrics from graph theory and network analysis (Estrada, 2012) e.g. centrality, connectivity, community detection, etc. (Hogan et al., 2020). In this prediction scenario, using the ontology is relevant since data need to follow the structure defined by it. Automatically generating the queries necessary to implement the methods of each predicted API path is also a challenge that still needs to be addressed.

- API version management. Ontology changes may affect its corresponding KG and as, a consequence, the API which allows consuming KG data. Therefore, new techniques are needed to detect ontology changes and propagate them into their corresponding APIs, ensuring that applications will not crash when the underlying ontology is updated. Existing work in ontology evolution (Osborne and Motta, 2018; Pernisch et al., 2020; Zablith et al., 2015) could be reused and extended to help meet this open research challenge.

- API generation from application requirements. Application developers may want to consume KG data for purposes that are different to those proposed or motivated when the ontology to represent such data was developed. Therefore, it is necessary to investigate alternatives to provide developers with the mechanisms to generate ad-hoc APIs to consume the data that they need for their applications. One alternative could be to allow developers reuse application use cases, requirements, types of users involved, etc., in order to generate the API paths, methods, and queries that are required for the application implementation. Several initiatives proposed to transform natural language into knowledge base queries (Diefenbach et al., 2018) can be used/adapted to generate the queries needed for implementing the ad-hoc API methods. Also, there is an opportunity to explore how language models (e.g. GPT-3 (Brown et al., 2020)) can be used in the translation of application requirements into API paths. 



\section{Bibliography}

Apache (2014). Apache Jena Fuseki. https://jena.apache.org/ documentation/fuseki2, Accessed: 2020-03-12. 20

Arwe, J., Speicher, S., and Malhotra, A. (2015). Linked Data Platform 1.0. W3C recommendation, W3C. https://www.w3.org/TR/2015/REC-ldp-20150226. 18

Badenes-Olmedo, C., Espinoza-Arias, P., and Corcho, O. (2021). R4R: Template-based REST API Framework for RDF Knowledge Graphs. In Proceedings of the ISWC 2021 Posters, Demos and Industry Tracks: From Novel Ideas to Industrial Practice co-located with 20th International Semantic Web Conference, Virtual Conference, 2021. CEUR Workshop Proceedings. 23

Berners-Lee, T., Capadisli, S., Verborgh, R., Kjernsmo, K., Bingham, J., and Zagidulin, D. (2019). The Solid Ecosystem, Editor's Draft. https://solid.github.io/ specification. Accessed: 2020-09-11. 19

Berners-Lee, T., Fielding, R., and Masinter, L. (1998). RFC2396: Uniform resource identifiers (URI): generic syntax. 103

Berrueta, D., Phipps, J., Miles, A., Baker, T., and Swick, R. (2008). Best Practice Recipes for Publishing RDF Vocabularies. Working group note, W3C. 2

Bray, T. et al. (2014). The JavaScript Object Notation (JSON) Data Interchange Format. 2

Brown, T. B., Mann, B., Ryder, N., Subbiah, M., Kaplan, J., Dhariwal, P., Neelakantan, A., Shyam, P., Sastry, G., Askell, A., et al. (2020). Language Models are Few-Shot Learners. arXiv preprint arXiv:2005.14165. 91 
Clark, K., Torres, E., and Feigenbaum, L. (2008). SPARQL Protocol for RDF. W3C recommendation, W3C. https://www.w3.org/TR/2008/ REC-rdf-sparql-protocol-20080115. 17

Corcho, O., Fernández-López, M., and Gómez-Pérez, A. (2003). Methodologies, tools and languages for building ontologies. Where is their meeting point? Data $\&$ knowledge engineering, 46(1):41-64. 1

Corradi, A., Foschini, L., and Ianniello, R. (2014). Linked Data for Open Government: The Case of Bologna. In 2014 IEEE Symposium on Computers and Communications (ISCC), pages 1-7. IEEE. 1

Cyganiak, R. and Bizer, C. (2007). Pubby - A Linked Data Frontend for SPARQL Endpoints. http://wifo5-03.informatik.uni-mannheim.de/pubby. Accessed: 2020-03-08. 20

Daga, E., Panziera, L., and Pedrinaci, C. (2015). A BASILar approach for building web APIs on top of SPARQL endpoints. In CEUR Workshop Proceedings, volume 1359, pages 22-32. 3, 21

Daquino, M., Heibi, I., Peroni, S., and Shotton, D. (2020). Creating Restful APIs over SPARQL endpoints with RAMOSE. arXiv preprint arXiv:200\%.160\%9. 24

Deliot, C. (2014). Publishing the British National Bibliography as Linked Open Data. Catalogue \& Index, 174:13-18. 1

Diefenbach, D., Lopez, V., Singh, K., and Maret, P. (2018). Core techniques of question answering systems over knowledge bases: a survey. Knowledge and Information systems, 55(3):529-569. 91

Epimorphics (2011). Elda: The linked-data API in Java. http://epimorphics. github.io/elda/index.html. Accessed: 2020-03-09. 20

Erling, O. and Mikhailov, I. (2009). RDF Support in the Virtuoso DBMS. In Networked Knowledge-Networked Media, pages 7-24. Springer. 20

Espinoza-Arias, P., Fernández-Ruiz, M. J., Morlán-Plo, V., Notivol-Bezares, R., and Corcho, O. (2020a). The Zaragoza's Knowledge Graph: Open Data to Harness the City Knowledge. Information, 11(3):129. 1, 70, 79 
Espinoza-Arias, P., Garijo, D., and Corcho, O. (2020b). Mapping the Web Ontology Language to the OpenAPI Specification. In International Conference on Conceptual Modeling, pages 117-127. Springer. 66

Espinoza-Arias, P., Garijo, D., and Corcho, O. (2021). Crossing the Chasm Between Ontology Engineering and Application Development: A Survey. Journal of Web Semantics, 70:100655. 16, 53

Estrada, E. (2012). The Structure of Complex Networks: Theory and Applications. Oxford University Press. 91

ETSI (2020). ETSI TS 103264 V3.1.1. SmartM2M; Smart Applications; Reference Ontology and oneM2M Mapping. Technical report. 79

Farré, C., Varga, J., and Almar, R. (2019). GraphQL schema generation for dataintensive web APIs. In International Conference on Model and Data Engineering, pages 184-194. Springer. 23

Feigenbaum, L., Torres, E., Clark, K., and Williams, G. (2013). SPARQL 1.1 Protocol. W3C recommendation, W3C. https://www.w3.org/TR/2013/ REC-sparql11-protocol-20130321. 17

Fernández-Izquierdo, A., Poveda-Villalón, M., and García-Castro, R. (2019). CORAL: A Corpus of Ontological Requirements Annotated with Lexico-Syntactic Patterns. In European Semantic Web Conference, pages 443-458. Springer. 89

Fernández-López, M., Gómez-Pérez, A., and Juristo, N. (1997). Methontology: from ontological art towards ontological engineering. 10

Fernández-Sellers, M. (2015). Linked Open Data Inspector. https://github.com/ marfersel/LODI. Accessed: 2020-03-12. 21

Fielding, R. T. and Taylor, R. N. (2002). Principled Design of the Modern Web Architecture. ACM Transactions on Internet Technology (TOIT), 2(2):115-150. 2 18

Fletcher, G., Groth, P., and Sequeda, J. (2020). Knowledge Scientists: Unlocking the data-driven organization. arXiv preprint arXiv:2004.0791\%. 2

Foundation, T. A. S. (2013). Apache Marmotta. http://marmotta.apache.org. Accessed: 2020-07-10. 21 
Garcia, A., O'Neill, K., Garcia, L. J., Lord, P., Stevens, R., Corcho, O., and Gibson, F. (2010). Developing Ontologies within Decentralised Settings. In Semantic eScience, pages 99-139. Springer. 1

Garijo, D. and Osorio, M. (2020). OBA: An Ontology-Based Framework for Creating REST APIs for Knowledge Graphs. In Pan, J. Z., Tamma, V., d'Amato, C., Janowicz, K., Fu, B., Polleres, A., Seneviratne, O., and Kagal, L., editors, The Semantic $W e b-I S W C$ 2020, pages 48-64, Cham. Springer International Publishing. 23

Garijo, D. and Poveda-Villalón, M. (2020). Best Practices for Implementing FAIR Vocabularies and Ontologies on the Web. Applications and Practices in Ontology Design, Extraction, and Reasoning, 49:39. 55

Gómez-Pérez, A., Fernández-López, M., and Corcho, O. (2006). Ontological Engineering: with examples from the areas of Knowledge Management, e-Commerce and the Semantic Web. Springer Science \& Business Media. xiii, 10

Google (2010a). Linked Data API. https://github.com/UKGovLD/ linked-data-api. Accessed: 2020-03-02. 17

Google (2010b). Puelia. https://code.google.com/archive/p/puelia-php. Accessed: 2020-03-09. 20.

GraphQL, Foundation (2015). GraphQL. https://spec.graphql.org/ June2018, Accessed: 2020-10-10. 3, 19

Groth, P., Loizou, A., Gray, A. J., Goble, C., Harland, L., and Pettifer, S. (2014). Apicentric linked data integration: The open phacts discovery platform case study. Journal of Web Semantics, 29:12 - 18. Life Science and e-Science. 3

Grüninger, M. and Fox, M. S. (1995). Methodology for the design and evaluation of ontologies. In Proceedings of the Workshop on Basic Ontological Issues in Knowledge Sharing. xiii, 8,9

Hammar, K. (2020). The OWL2OAS Converter. https://github.com/ RealEstateCore/OWL2OAS, Accessed: 2020-06-15. 23

Hartig, O., Zhao, J., and Mühleisen, H. (2010). Automatic integration of metadata into the web of linked data. In Proceedings of the Demo Session at the 2nd Workshop on Trust and Privacy on the Social and Semantic Web (SPOT) at ESWC, pages 2-4. 20] 
Hendler, J., Holm, J., Musialek, C., and Thomas, G. (2012). US Government Linked Open Data: Semantic.data.gov. IEEE Intelligent Systems, (3):25-31. 1

Heyvaert, P., De Meester, B., Pandit, H., and Verborgh, R. (2020). Walder. https: //github.com/KNowledgeOnWebScale/walder. Accessed: 2020-10-20. 24

Hogan, A., Blomqvist, E., Cochez, M., d'Amato, C., de Melo, G., Gutierrez, C., Gayo, J. E. L., Kirrane, S., Neumaier, S., Polleres, A., et al. (2020). Knowledge Graphs. arXiv preprint arXiv:2003.02320. 1, 91

Initiative, O. (2011). OpenAPI Specification. https://swagger.io/ specification. Accessed: 2020-10-05. 19

Jansen, G., Coburn, A., Soroka, A., and Marciano, R. (2019). Using Data Partitions and Stateless Servers to Scale Up Fedora Repositories. In 2019 IEEE International Conference on Big Data (Big Data), pages 3098-3102. IEEE Computer Society. 22

Jin, B., Sahni, S., and Shevat, A. (2018). Designing Web APIs: Building APIs That Developers Love. O'Reilly Media, Inc. 51

Jusevicius, M. (2014). Graphity: Generic processor for declarative linked data applications. In SWAT4LS. 22

Jusevičius, M. (2016). Linked Data Templates. In Proceedings of the XML London conference, pages 50-55. 18

Jusevičius, M. (2016). AtomGraph Processor. https://github.com/AtomGraph/ Processor, Accessed: 2020-08-15. 22

Keet, M. (2018). An Introduction to Ontology Engineering, volume 1. Maria Keet. 1

Kellogg, G., Lanthaler, M., and Sporny, M. (2014). JSON-LD 1.0. W3C recommendation, W3C. https://www.w3.org/TR/2014/REC-json-ld-20140116. 27

Kotis, K. I., Vouros, G. A., and Spiliotopoulos, D. (2020). Ontology engineering methodologies for the evolution of living and reused ontologies: status, trends, findings and recommendations. The Knowledge Engineering Review, 35. 2

Lanthaler, M. and Gütl, C. (2013). Hydra: A Vocabulary for Hypermedia-Driven Web APIs. $L D O W, 996.18$ 
Lehmann, J., Isele, R., Jakob, M., Jentzsch, A., Kontokostas, D., Mendes, P. N., Hellmann, S., Morsey, M., Van Kleef, P., Auer, S., et al. (2015). DBpedia - A Largescale, Multilingual Knowledge Base Extracted from Wikipedia. Semantic web, 6(2):167-195. 1

Lin, C.-Y. (2004). ROUGE: A Package For Automatic Evaluation of Summaries. In Text summarization branches out, pages 74-81. 82

Lisena, P., Meroño-Peñuela, A., Kuhn, T., and Troncy, R. (2019). Easy Web API Development with SPARQL Transformer. In Ghidini, C., Hartig, O., Maleshkova, M., Svátek, V., Cruz, I., Hogan, A., Song, J., Lefrançois, M., and Gandon, F., editors, The Semantic Web - ISWC 2019, pages 454-470, Cham. Springer International Publishing. 22

Malone, J., Brown, A., Lister, A. L., Ison, J., Hull, D., Parkinson, H., and Stevens, R. (2014). The Software Ontology (SWO): a resource for reproducibility in biomedical data analysis, curation and digital preservation. Journal of biomedical semantics, 5(1):25. BioMed Central. 79

Masse, M. (2011). REST API Design Rulebook: Designing Consistent RESTful Web Service Interfaces. O'Reilly Media, Inc. 103

Mayer, S., Hodges, J., Yu, D., Kritzler, M., and Michahelles, F. (2017). An Open Semantic Framework for the industrial Internet of Things. IEEE Intelligent Systems, 32(1):96-101. 22

McGuinness, D. L., Van Harmelen, F., et al. (2004). OWL Web Ontology Language Overview. W3C recommendation, 10(10):2004. 2

Meroño-Peñuela, A. and Hoekstra, R. (2016). grlc Makes GitHub Taste Like Linked Data APIs. In European Semantic Web Conference, pages 342-353. Springer. 3, 21

Miller, J. J. (2013). Graph Database Applications and Concepts with Neo4j. In Proceedings of the Southern Association for Information Systems Conference, Atlanta, GA, USA, volume 2324. 20

Muñoz-Soro, J. F., Esteban, G., Corcho, O., and Serón, F. (2016). Pproc, an ontology for transparency in public procurement. Semantic Web, 7(3):295-309. 79

Navigli, R. and Ponzetto, S. P. (2010). Babelnet: Building a very large multilingual semantic network. In Proceedings of the 48th annual meeting of the association for computational linguistics, pages 216-225. 90 
Noy, N., Gao, Y., Jain, A., Narayanan, A., Patterson, A., and Taylor, J. (2019). Industry-scale Knowledge Graphs: Lessons and Challenges. Queue, 17(2):48-75. 1

Ogbuji, C. (2013). SPARQL 1.1 Graph Store HTTP protocol. W3C recommendation, W3C. https://www.w3.org/TR/2013/ REC-sparql11-http-rdf-update-20130321. 17

Ontotext (2015). GraphDB. https://graphdb.ontotext.com. Accessed: 202003-12. 20

Osborne, F. and Motta, E. (2018). Pragmatic Ontology Evolution: Reconciling User Requirements and Application Performance. In International Semantic Web Conference, pages 495-512. Springer. 91

Parkkila, J., Radulovic, F., Garijo, D., Poveda-Villalón, M., Ikonen, J., Porras, J., and Gómez-Pérez, A. (2017). An ontology for videogame interoperability. Multimedia tools and applications, 76(4):4981-5000. 79

Pernisch, R., Dell'Aglio, D., Serbak, M., and Bernstein, A. (2020). ChImp: Visualizing Ontology Changes and theirImpact in Protégé. In Fifth International Workshop on Visualization and Interaction for Ontologies and Linked Data, pages 47-60. CEUR Workshop Proceedings. 91

Peroni, S. (2016). A simplified agile methodology for ontology development. In Proceedings of the 13th OWL: Experiences and Directions Workshop and 5th OWL reasoner evaluation workshop, Bologna, Italy, November 20, 2016, pages 55-69. Springer. xiii, 14

Piñero, J., Ramírez-Anguita, J. M., Saüch-Pitarch, J., Ronzano, F., Centeno, E., Sanz, F., and Furlong, L. I. (2020). The DisGeNET knowledge platform for disease genomics: 2019 update. Nucleic acids research, 48(D1):D845-D855. 1

Postman (2020). State of the API Report. https://www.postman.com/ state-of-api. Accessed: 2021-03-22. 27

Poveda-Villalón, M., Fernández-Izquierdo, A., and García-Castro, R. (2019). Linked Open Terms (LOT) Methodology. https://doi.org/10.5281/zenodo. 2539305, xiii, 15, 16, 50

Richardson, L., Amundsen, M., and Ruby, S. (2013). RESTful Web APIs: Services for a Changing World. O'Reilly Media, Inc. 103 
Safris, S. (2019). A Deep Look at JSON vs. XML, Part 1: The History of Each Standard. https://www.toptal.com/web/json-vs-xml-part-1. Accessed: 2021-03-25. 27

Salvadori, I. and Siqueira, F. (2015). A Maturity Model for Semantic RESTful Web APIs. In 2015 IEEE International Conference on Web Services, pages 703-710. IEEE. 3

Schröder, M., Hees, J., Bernardi, A., Ewert, D., Klotz, P., and Stadtmüller, S. (2018). Simplified sparql rest api. In Gangemi, A., Gentile, A. L., Nuzzolese, A. G., Rudolph, S., Maleshkova, M., Paulheim, H., Pan, J. Z., and Alam, M., editors, The Semantic Web: ESWC 2018 Satellite Events, pages 40-45, Cham. Springer International Publishing. 3

Seaborne, A. and Harris, S. (2013). SPARQL 1.1 Query Language. W3C Recommendation, W3C. https://www.w3.org/TR/2013/ REC-sparql11-query-20130321, 2

Semantic Integration Ltd., h. (2018). HyperGraphQL. https://www. hypergraphql.org. Accessed: 2021-05-13. 63

Severance, C. (2012). Discovering JavaScript Object Notation. Computer, 45(4):6-8. 27

Shadbolt, N., O'Hara, K., Berners-Lee, T., Gibbins, N., Glaser, H., Hall, W., et al. (2012). Linked Open Government Data: Lessons from Data.gov.uk. IEEE Intelligent Systems, 27(3):16-24. 1

Shefchek, K. A., Harris, N. L., Gargano, M., Matentzoglu, N., Unni, D., Brush, M., Keith, D., Conlin, T., Vasilevsky, N., Zhang, X. A., et al. (2020). The Monarch Initiative in 2019: An integrative data and analytic platform connecting phenotypes to genotypes across species. Nucleic acids research, 48(D1):D704-D715. 1

Simon, A., Wenz, R., Michel, V., and Di Mascio, A. (2013). Publishing bibliographic records on the web of data: Opportunities for the bnf (french national library). In Extended Semantic Web Conference, pages 563-577. Springer. 1

Staab, S., Studer, R., Schnurr, H.-P., and Sure, Y. (2001). Knowledge processes and ontologies. IEEE Intelligent systems, 16(1):26-34. IEEE. xiii, 11 
Stasiewicz, A. and Waqar, M. (2016). Deliverable 3.2: Report on OpenGovIntelligence ICT tools - first release. Technical report, National University of Ireland (NUIG). OpenGovIntelligence Project. 22

Studer, R., Benjamins, V. R., and Fensel, D. (1998). Knowledge engineering: Principles and methods. Data and Knowledge Engineering, 25(1-2):161-197. Elsevier. 1]

Suárez-Figueroa, M. C., Gómez-Pérez, A., and Fernandez-Lopez, M. (2015). The neon methodology framework: A scenario-based methodology for ontology development. Applied ontology, 10(2):107-145. xiii, 12,13

SYSTAP, L. (2015). Blazegraph. https://blazegraph.com. Accessed: 2020-03-12. 20

Taelman, R., Van Herwegen, J., Vander Sande, M., and Verborgh, R. (2018a). Comunica: a Modular SPARQL Query Engine for the Web. In Proceedings of the 17th International Semantic Web Conference. 24

Taelman, R., Van Herwegen, J., Vander Sande, M., and Verborgh, R. (2018b). Comunica: a modular sparql query engine for the web. In International Semantic Web Conference, pages 239-255. Springer. 63

Taelman, R., Vander Sande, M., and Verborgh, R. (2018c). Graphql-ld: linked data querying with graphql. In ISWC2018, the 17th International Semantic Web Conference, pages 1-4. 24,63

TopQuadrant (2001). TopBraid. https : / www . topquadrant . com. Accessed: 202105-13. 63

Torres, E., Clark, K., and Feigenbaum, L. (2008). SPARQL protocol for RDF. W3C recommendation, W3C. https://www.w3.org/TR/2008/ REC-rdf-sparql-protocol-20080115, 26

Van Herwegen, J., Taelman, R., and Verborgh, R. (2020). Community Solid Server. https://github.com/solid/community-server. Accessed: 2020-11-20. 24

Verborgh, R. and Vander Sande, M. (2020). The Semantic Web identity crisis: in search of the trivialities that never were. Semantic Web, (Preprint):1-9. 2

Vila-Suero, D., Villazón-Terrazas, B., and Gómez-Pérez, A. (2013). datos.bne.es: A library linked dataset. Semantic Web, 4(3):307-313. 1 
Vrandečić, D. and Krötzsch, M. (2014). Wikidata: a free collaborative knowledgebase. Communications of the ACM, 57(10):78-85. 1

Wiśniewski, D., Potoniec, J., and Ławrynowicz, A. (2021). Bigcq: A large-scale synthetic dataset of competency question patterns formalized into sparql-owl query templates. arXiv preprint arXiv:2105.09574. 89

Wiśniewski, D., Potoniec, J., Ławrynowicz, A., and Keet, C. M. (2019). Analysis of Ontology Competency Questions and their formalizations in SPARQL-OWL. Journal of Web Semantics, 59:100534. 89

Zablith, F., Antoniou, G., d'Aquin, M., Flouris, G., Kondylakis, H., Motta, E., Plexousakis, D., and Sabou, M. (2015). Ontology evolution: a process-centric survey. The knowledge engineering review, 30(1):45-75. 91

Zaveri, A., Dastgheib, S., Wu, C., Whetzel, T., Verborgh, R., Avillach, P., Korodi, G., Terryn, R., Jagodnik, K., Assis, P., and Dumontier, M. (2017). smartAPI: Towards a More Intelligent Network of Web APIs. In Blomqvist, E., Maynard, D., Gangemi, A., Hoekstra, R., Hitzler, P., and Hartig, O., editors, The Semantic Web, pages 154-169, Cham. Springer International Publishing. 63

Zeginis, D., Kalampokis, E., Roberts, B., Moynihan, R., Tambouris, E., and Tarabanis, K. A. (2017). Facilitating the Exploitation of Linked Open Statistical Data: JSONQB API Requirements and Design Criteria. In HybridSemStats@ ISWC. 22 


\section{ANNEX A}

\section{REST APIs resource naming strategy}

REST APIs follow a resource identifier design (aka resource naming) strategy with URIs. A generic URI syntax, according to (Berners-Lee et al., 1998), has the following structure: URI = scheme "://" authority "/" path [ "?" query ] [ "\#" fragment ]. In this structure, scheme refers to a specification to assign identifiers within that scheme. Authority concerns to the naming authority which governs the name space defined in the remainder of the URI. Path regards to data (usually organized in hierarchical form) that identifies a resource within the scheme and the authority. Query refers to a set of optional parameters to identify the resource or provide additional capabilities. Finally, the optional fragment component allows to identify a secondary resource by reference.

\section{A.1 Rules for naming REST APIs}

Unlike ontology naming strategies, that do not follow a unified convention, in REST APIs the rules for naming resources are clearly specified, discussed and exemplified in several works. However, we provide a summary of the main decisions, provided in (Masse, 2011) and (Richardson et al., 2013), that should be considered when naming resources for KG data consumption, i.e. to define the path component of the URI namely API path. This path terminates when there is a question mark (?), a hash character $(\#)$, or by the end of the URI. Next, we present the most relevant rules adopted for the API path design whether it is done manually or automated. For illustrating purposes, we will use an example API throughout this annex with the following URI scheme and 
authority components: http://api.example.com; however, we will omit to include this part in the examples provided.

1. Use lowercase letters for resource paths. For example, the path of a collection of countries / countries

2. Use forward slash separator (/) to indicate a hierarchical relationship. In addition, avoid using this separator at the end of the resource. For example, countries contain provinces therefore the path would be /countries/provinces

3. Use singular nouns for naming single resources and plural nouns for collections of resources. For example, the resource path of a collection of countries would be / countries and for a single country would be /spain

4. Use hyphens (-) to improve the readability and avoid using underscores (_). For example, to represent the Czech Republic resource path would be /czechrepublic

5. Use URI templates to include path segments that must be substituted before resolution. For example, /countries/\{countryId\} contains the template \{countryId\} that will be substituted by the REST API or its clients, and will adopt a specific numeric or alphanumeric identifier value like / countries/1234 which refers to a country with ID 1234.

6. Do not include file extensions. Instead, manage a content negotiation strategy to provide the requested files accordingly.

7. Do not include CRUD operation names. Instead, use HTTP request methods to indicate what function should be performed.

In addition, APIs use to include a query component. This component allows users to achieve the following functionality:

1. Filtering collections of resources. For example, the call GET / countries? inha bitants-number $>=10000000$ requests for countries with inhabitants number major or equal to 10000000 .

2. Paginating collection results. For example, the call GET / countries?pageSi $z e=25$ \&page Start Index $=50$ will get back maximum 25 countries in the response (specified in the pagesize parameter) and the first in the response will be the fifty country (defined in the pageStartIndex parameter). In addition, 
other parameters can be specified to manage pagination, for example, offset to define the beginning page of results, limit to specify the maximum number of results in a specific page, cursor to indicate what is the resource from which the request should begin, among others.

3. Sorting collection results. For example, the call GET / countries?sort=in habitants-number will bring back the countries ordered by inhabitants number. In addition, the sorting can be configured as ascendant or descendant, if desired.

\section{A.2 Rules for naming REST APIs based on ontology arte- facts}

Once we have explained the common practices for naming REST APIs, we propose additional rules that should be adopted to generate APIs based on ontology artefacts. To illustrate these rules we will use the Video Game Ontology 47 and its competency questions 48

1. Use the ontology class label to name the API paths. For example, the CQ "List all players" would have the API path / players because the answer is represented by the class with label Player. Note that the path is in plural and lowercase as recommended in the common practices.

2. When the CQ involves more than one ontology class take into account the following alternatives:

(a) If the CQ regards to two ontology classes and there is a directed relation between them, take the ontology domain class of this relation as the first level of the resource hierarchy and its range as the next level. For example, the CQ "What games has the player played?" would have the API path /players/ player-id\}/games because the answer is represented by the object property has played with domain Player and range Game. Note that there is needed the $\{$ player-id\} template because the CQ ask for a specific player.

(b) If the CQ regards to two ontology classes and there is not a directed relation between them the following scenarios can occur:

${ }^{47}$ http://vocab.linkeddata.es/vgo

${ }^{48}$ https://doi.org/10.5281/zenodo.1967306 
i. There are several classes and relations between them that allow finding a directed walk from the origin and target class. Therefore, define the API path considering only the origin and target ontology classes. For example, the CQ "What are the items of a player?" would have the API path /players/\{player-id\}/items. Despite the walk to solve the CQ involves the relationships Player ownscharacter Character and Character ownsItem Item, the API path considers only the start and end classes of the solution walk.

ii. If there is an inverse relation between the origin and target classes, the $\mathrm{CQ}$ can be solved because this relation allows defining a SPARQL query to get the requested data. Therefore, define the API path taking only the origin class and use the target one to specify a query parameter. For example, the CQ "List all games of a certain genre" would have the API path /games?genre=\{genre-id $\}$ because there is not a directed relation between Genre and Game classes but there is the inverse relation has genre between Game and Genre. The template \{genre-id\} should be substituted by the id value of the required genre.

3. When the origin and target are the same ontology class and there is a relation between them, define the API path as explained in rule 2a. In addition, insert the label of this relation in the path and between the classes it relates. For example, the CQ "What are the friends of a player?" would have the API path /players/\{player-id\}/is-friend-with-player/players because the class $\mathrm{Pl}$ layer is related to itself to describe that a player has friends that are also players (this relation is represented by the object property is friend with player). Note that there is needed the $\{$ player-id\} template because the $\mathrm{CQ}$ ask for a specific player.

4. Use ontology property labels to define the names of filter parameters for those cases when the CQs define specific criteria to search for a resource. For example, the CQ "What are the games released after 5 January 2010?" would have the API path /games?release-date>"05/01/2010" because the class Game has the datatype property release date that represents when the game was released.

5. When the ontology elements do not have labels (for example, when the ontology reuse elements by referencing to other ontology) use the URI fragment of the ontology element to name the API path or query component, and format the 
label according to the common practices. Use the URI fragment only if it is not an opaque URI.

6. Avoid defining nested resources with more than two nested levels. 



\section{ANNEX B}

\section{API Specification Template}

\begin{tabular}{|l|l|}
\hline \multicolumn{2}{|l|}{ General information } \\
\hline API name & \\
\hline API version & \\
\hline Base URL & \\
\hline API description & \\
\hline License & \\
\hline Authorization & \\
\hline Contact & \\
\hline
\end{tabular}

\begin{tabular}{|l|l|l|l|}
\hline \multicolumn{4}{|l|}{ Details of each API path } \\
\hline API path & Operation & Input & Output \\
\hline & & & \\
\hline & & & \\
\hline & & & \\
\hline & & & \\
\hline
\end{tabular}

\begin{tabular}{|c|c|c|}
\hline \multicolumn{3}{|c|}{ Status codes } \\
\hline Code & Description & Detail \\
\hline & & \\
\hline & & \\
\hline & & \\
\hline
\end{tabular}





\section{ANNEX C}

\section{Questionnaire on generating REST APIs from ontology artefacts}

We would invite you to participate in this questionnaire which will allow investigating new mechanisms to make it easier for application developers to consume data represented with ontologies. More specifically, we want to know the perception of developers regarding API paths which allow getting data to solve ontology requirements. It will take around 25 minutes to fill out this questionnaire. All answers are anonymous.

You will be asked to provide your opinion and some short answers about different aspects of a set of API paths we will show you. The questionnaire is divided into the following main aspects:

- Background

- Specific questions on REST APIs

- Specific questions on API paths based on ontology artefacts

Take into account some of the existing best practices to build REST API paths that should be followed:

- Use singular nouns for naming single resources and plural nouns for collections of resources. For example, the resource path of a collection of countries would be /countries and for a single country resource would be /spain.

- Use URI templates to include path segments that must be substituted before resolution. For example, /countries/\{countryId\} contains the template $\{$ countryId $\}$ that will be substituted by the REST API or its clients, and will adopt a specific 
numeric or alphanumeric identifier value like/countries/1234 which refers to a country with identifier 1234 .

- APIs usually include a query component that allows achieving certain functionality. For example, /countries?inhabitants-number $=10000000$ requests for countries with inhabitants number equal to 10000000 .

\section{C.1 Background}

1. Are you familiar with Web Services?

$\square$ yes, but I have only used them

$\square$ yes, I have designed, implemented, and used them

$\square$ no

2. Are you familiar with REST APIs?

es, but I have only used them for data consumption

$\square$ yes, I have designed, implemented, and used them

$\square$ no

3. Are you familiar with GraphQL?

$\square$ es, but I have only used them for data consumption

$\square$ yes, I have designed the schemes and queries needed to consume data

$\square$ no

4. How many Web APIs have you developed?
$\square 0$
between 1 and 5
between 6 and 10
$\square$ more than 10

5. Have you used any semantic technology (e.g. RDF, SPARQL, OWL, among others)??
$\square$ yes
$\square$ no 
6. If the answer to the previous question was yes, please specify the semantic technologies you are used to:

\section{C.2 Specific questions regarding REST APIs}

1. Executing the GET operation on the API path / resources would return:

$\square$ a list of resources

$\square$ the information of a specific resource

$\square$ a list of resources that match the search paremeter value

2. Executing the GET operation on the API path /resources/\{resources-id\} would return:

$\square$ a list of resources

$\square$ the information of a specific resource

$\square$ a list of resources that match the search paremeter value

3. Executing the GET operation on the API path /resources?query="value" would return:

a list of resources

the information of a specific resource

a list of resources that match the search paremeter value

\section{C.3 Specific questions on APIs based on ontology artefacts}

Next, given a domain, we present a set of requirements that have been gathered to build the ontology that represents this domain. Then, for each requirement (written in natural language) we present the API paths that will allow obtaining data to solve it. In each case, assume that the operation to be executed in the API path is a GET.

In the following sections, we will present the requirements and API paths of three different domains. In addition, a set of questions regarding these API paths.

\section{C.3.1 First domain: Local business census and activities licenses.}

This ontology represents data related to the census of business premises and terraces of a municipality, as well as their associated economic activities and opening licenses. The 
documentation of this ontology is available at http://vocab.ciudadesabiertas. es/def/comercio/tejido-comercial/index-en.html.

- For the requirement: "What are the local businesses of the city?"

Consider the following API paths:

1) /local-business/local-business

2) /local-businesses

1. From the API paths presented it could be said that:

$\square$ The 1st is generated automatically and the 2nd is generated manually

$\square$ The 2 nd is generated automatically and the 1st is generated manually

$\square$ Both are generated manually

$\square$ Both are automatically generated

$\square$ It is not possible to determine how they were generated (Indistinguishable)

2. Which API path would you prefer to use?

$\square$ /local-business/local-business

$\square$ /local-businesses

$\square$ Both

$\square$ I don't have a preference

- For the requirement: "Obtain the information of a certain terrace"

Consider the following API paths:

1) /local-business/terrace/\{id $\}$

2) $/$ terraces $/\{$ terrace-id $\}$

1. From the API paths presented it could be said that:

$\square$ The 1 st is generated automatically and the 2 nd is generated manually

The 2 nd is generated automatically and the 1st is generated manually

Both are generated manually

Both are automatically generated

$\square$ It is not possible to determine how they were generated (Indistinguishable)

2. Which API path would you prefer to use? 
$\square /$ local-business/terrace/ $\{$ id $\}$

$\square /$ terraces $/\{$ terrace-id $\}$

$\square$ Both

$\square$ I don't have a preference

- For the requirement: "List all the shopping areas of the city." Consider the following API paths:

1) /local-business/shopping-area

2) /shopping-areas

1. From the API paths presented it could be said that:

The 1st is generated automatically and the 2 nd is generated manually

$\square$ The 2nd is generated automatically and the 1st is generated manually

$\square$ Both are generated manually

Both are automatically generated

$\square$ It is not possible to determine how they were generated (Indistinguishable)

2. Which API path would you prefer to use?

$\square$ /local-business/shopping-area

$\square$ /shopping-areas

$\square$ Both

I don't have a preference

\section{C.3.2 Second domain: Air Quality}

This ontology represents the air quality observations that are being published by many organizations and individuals as a result of the measurements that are taken by air quality stations and sensors. The documentation of this ontology is available at http:// vocab.linkeddata.es/datosabiertos/def/medio-ambiente/calidad-aire/ index-en.html

- For the requirement: "What are the air quality stations?"

Consider the following API paths:

1)/service/air-quality/list

2) /air-quality-stations 
1. From the API paths presented it could be said that:

$\square$ The 1st is generated automatically and the 2nd is generated manually

$\square$ The 2nd is generated automatically and the 1st is generated manually

$\square$ Both are generated manually

$\square$ Both are automatically generated

$\square$ It is not possible to determine how they were generated (Indistinguishable)

2. Which API path would you prefer to use?

$\square$ /service/air-quality/list

$\square$ /air-quality-stations

$\square$ Both

$\square$ I don't have a preference

Eor the requirement: "What is the information of an air quality station?"

Consider the following API paths:

1) /service/air-quality/list?id="value"

2) /air-quality-stations/ \{air-quality-station-id\}

1. From the API paths presented it could be said that:

The 1st is generated automatically and the 2nd is generated manually

$\square$ The 2nd is generated automatically and the 1st is generated manually

Both are generated manually

$\square$ Both are automatically generated

$\square$ It is not possible to determine how they were generated (Indistinguishable)

2. Which API path would you prefer to use?

$\square$ /service/air-quality/list?id="value"

$\square$ /air-quality-stations/\{air-quality-station-id\}

$\square$ Both

$\square$ I don't have a preference

- For the requirement: "What are the air quality observations?" 
Consider the following API paths:

1) /service/air-quality

2) /air-quality-observations

1. From the API paths presented it could be said that:

The 1 st is generated automatically and the 2 nd is generated manually

$\square$ The 2nd is generated automatically and the 1st is generated manually

$\square$ Both are generated manually

Both are automatically generated

$\square$ It is not possible to determine how they were generated (Indistinguishable)

2. Which API path would you prefer to use?

$\square$ /service/air-quality

$\square$ /air-quality-observations

$\square$ Both

$\square$ I don't have a preference

\section{C.3.3 Third domain: Public Procurement}

This ontology defines the concepts to describe the public procurement process and the contracts of the public sector. The documentation of this ontology is available at http://contsem.unizar.es/def/sector-publico/pproc.html

- For the requirement: "List all contracts of the city"

Consider the following API paths:

1) / service/public-procurement/contract

2) / contracts

1. From the API paths presented it could be said that:

$\square$ The 1st is generated automatically and the 2 nd is generated manually

$\square$ The 2nd is generated automatically and the 1st is generated manually

Both are generated manually

Both are automatically generated

It is not possible to determine how they were generated (Indistinguishable) 
2. Which API path would you prefer to use?

$\square$ /service/public-procurement/contract

$\square /$ contracts

$\square$ Both

$\square$ I don't have a preference

- For the requirement: "What are the details of a contracting organization?"

Consider the following API paths:

1) / service/public-procurement/contracting-body/\{id $\}$

2) /organizations/ \{organization-id\}

1. From the API paths presented it could be said that:

$\square$ The 1st is generated automatically and the 2nd is generated manually

$\square$ The 2nd is generated automatically and the 1st is generated manually

Both are generated manually

$\square$ Both are automatically generated

$\square$ It is not possible to determine how they were generated (Indistinguishable)

2. Which API path would you prefer to use?

$\square$ /service/public-procurement/contracting-body/ $\{\mathrm{id}\}$

$\square$ /organizations/\{organization-id\}

$\square$ Both

I don't have a preference

— For the requirement: "What are the details of a notice?"

Consider the following API paths:

1) /service/public-procurement/notice/ \{noticeId $\}$

2) $/$ notices $/\{$ notice-id $\}$

1. From the API paths presented it could be said that:

The 1st is generated automatically and the 2nd is generated manually

$\square$ The 2nd is generated automatically and the 1st is generated manually 
Both are generated manually

$\square$ Both are automatically generated

$\square$ It is not possible to determine how they were generated (Indistinguishable)

2. Which API path would you prefer to use?

$\square$ /service/public-procurement/notice/\{noticeId

$\square /$ notices $/\{$ notice-id $\}$

$\square$ Both

$\square$ I don't have a preference 